\title{
Stream Flow and Analysis Study
}

by

D. G. Jackson

Westinghouse Savannah River Company

Savannah River Site

Aiken, South Carolina 29808

Lockwood Greene

RECEIVED

ОСТ 021997

O STI

DETRIBUTION OF TIIS DOCUMENT IS UNLMITSO MASTER

DOE Contract No. $D E-A C 09-765$ P $0000 /$

This paper was prepared in connection with work done under the above contract number with the U.S. Department of Energy. By acceptance of this paper, the publisher and/or recipient acknowledges the U. S. Government's right to retain a nonexclusive, royalty-free license in and to any copyright covering this paper, along with the right to reproduce and to authorize others to reproduce all or part of the copyrighted paper. 


\section{DISCLAIMER}

This report was prepared as an account of work sponsored by an agency of the United States Government. Neither the United States Government nor any agency thereof, nor any of their employees, makes any warranty, express or implied, or assumes any legal liability or responsibility for the accuracy, completeness, or usefulness of any information, apparatus, product, or process disclosed, or represents that its use would not infringe privately owned rights. Reference herein to any specific commercial product, process, or service by trade name, trademark, manufacturer, or otherwise does not necessarily constitute or imply its endorsement, recommendation, or favoring by the United States Government or any agency thereof. The views and opinions of authors expressed herein do not necessarily state or reflect those of the United States Government or any agency thereof.

This report has been reproduced directly from the best available copy.

Available to DOE and DOE contractors from the Office of Scientific and Technical Information, P. O. Box 62, Oak Ridge, TN 37831; prices available from (423) 576-8401.

Available to the public from the National Technical Information Service, U. S. Department of Commerce, 5285 Port Royal Road, Springfield, VA 22161. 


\section{DISCLAIMER}

Portions of this document may be illegible electronic image products. Images are produced from the best available original document. 


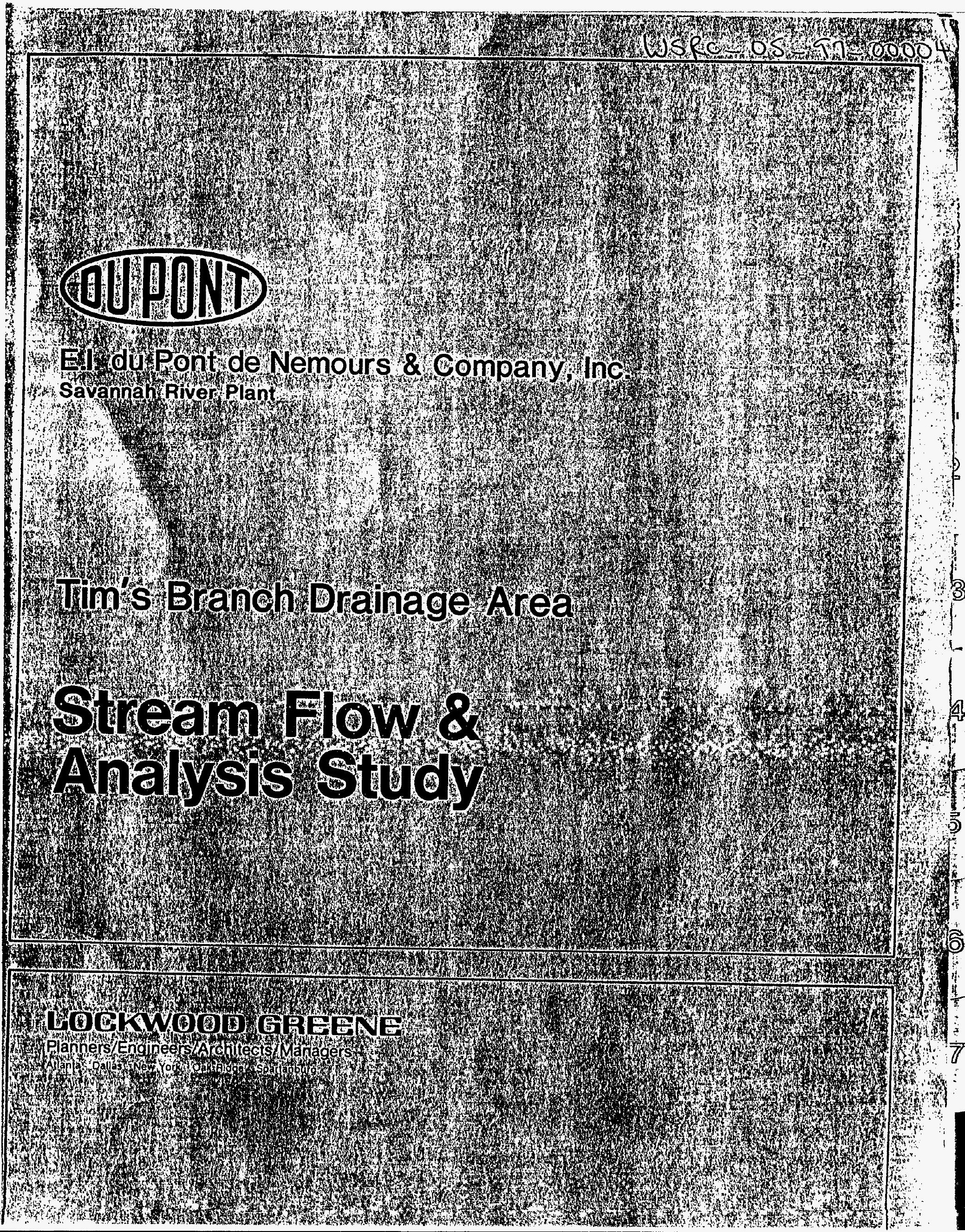


E. 1. DUPONT DE NEMOURS \& CO., INC.

SAVANMAH RIVER PLANT

AIKEN, SOUTH CAROLINA

STREAM FLOW AND AMALYSIS STUDY

FINAL REPORT

NOVEMBER 4, 1983

LOCKWOOD GREENE ENGINEERS, INC.

ATLANTA, GEORGIA

PROJECT NO. 83251.04

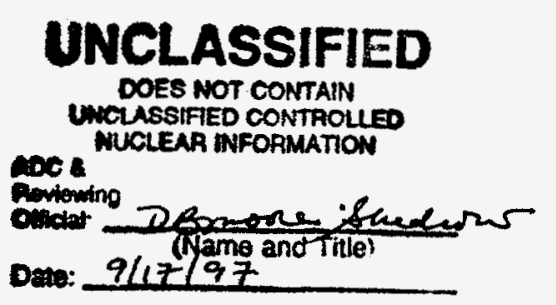




\section{LOCKWOOD GREENE}

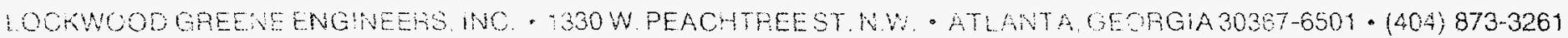

83251.04

November 3,1983

Mr. H. Drannon

E. 1. du Pont Company

Savannah River Plant

Aiken, South Carolina 29808

Dear Mr. Drannon:

Please find enclosed the final report entitled 'Tim's Branch Drainage Area - Stream Flow \& Analysis Study". The report is complete with the exception of TTO (total toxic organics) analyses, which are in progress at this time. The results of TTO analyses will be forwarded under separate cover as soon as they become available.

We appreciate the cooperation you and your associates have extended to us during the course of this study.

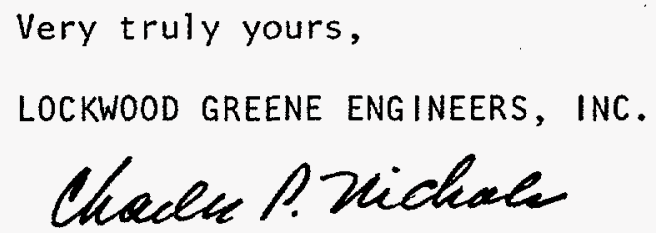

Charles P. Nichols, P.E. Project Manager

$C P N / P C$

Enclosure 


\section{STREAM FLOW AND ANALYSIS STUDY}

\section{TABLE OF CONTENTS}

\begin{tabular}{clc} 
Section No. & Title & Page No. \\
\hline 1 & Introduction & $1-1$ \\
2 & Scope of Services & $2-1$ \\
3 & Summary and Conclusions & $3-1$ \\
4 & Description of Study Area & $4-1$ \\
& A. Drainage Area & $4-1$ \\
& B. Stream Classification & $4-2$ \\
5 & Stream Flow and Analytical Results & $5-1$ \\
& A. Flow Data Analyses & $5-1$ \\
6 & B. Chemical Analion of Results & $5-5$ \\
& Evaluation for Tim's Branch & $6-1$ \\
7 & A. 7 Q 10 folytical Results & $6-1$ \\
& B. Appendix .
\end{tabular}
A. Tim's Branch Drainage Area Map
B. Sampling Site Locations
C. South Carolina Water Classification Standards reprint remored.
D. Dam and Weir Design Sketches
E. Flow Measurement Data
F. 24 Hour Flow Monitoring Narrative
G. Rainfall Data
H. USGS Station Flow Data
I. Analytical Results
J. 7 Q 10 Calculation
K. USGS Tim's Branch Drainage Basin Area 


\section{SECTION 1 - INTRODUCTION}

Lockwood Greene Engineers, Inc. (LGE) was retained by E. I. duPont de Nemours and Co., Inc., Savannah River Plant, Aiken, South Carolina, to conduct on-site flow measurements and sampling of tributaries and outfalls flowing into a portion of Tim's Branch Creek. Water samples were analyzed for chemical characteristics.

This report presents the results of the flow and analytical data collected during the 24 hour monitoring period, October 5 and 6, 1983. 


\section{SECTION 2 - SCOPE OF SERVICES}

\section{Objectives}

The objectives of the stream flow and analysis study were as follows:

1. Measure the amount of non-process water flow discharging into Tim's Branch for the purpose of calculating the dilution ratio of future process wastewater effluent with non-process water in Tim's Branch.

2. Calculate the 7 Q 10 flow* of Tim's Branch based on available data from the U.S. Geological Survey and on-site flow measurements.

3. Characterize the dry weather composition of stream and non-process water discharge points by means of a 24-hour composite and grab sampling program at five different points sampled simultaneously.

$* 7$ Q 10 is the lowest 7 day average flow expected on an average of once in 10 years.

Scope of Services

Specifically, the following items were included to accomplish the above objectives: 
1. Two days of field observations to determine optimal locations of flow measuring points for the purpose of designing dams and flow measuring devices.

2. Design of four flow measurement structures; fabrication and installation was by others (TET Construction Group at S.R.P.)

3. Specifications and installation sketches of flow indicators/recorders as required for five flow monitoring stations, described as follows:

Site 1: Tim's Branch tributary.

Site: 2: Garage/storm sewer outfall to Tim's Branch tributary.

Site 3: Cooling water/Sfor sewer outfall to Tim's Branch tributary.

Site 4: Tim's Branch downstream of Steed's Pond.

Site 5: Tim's Branch upstream of Site 1 above.

4. Four days of on-site consultation and inspection of flow monitoring stations during installation.

5. One set of dry weather 24-hour flow measurements made at five monitoring stations.

6. Review and compilation of meteorological data, USGS data, and calculation of Tim's Branch 7 Q 10 flow. 
7. One set of flow proportional 24-hour composite samples and 4-hour spaced grab samples for the five sampling locations during dry weather conditions.

8. Chemical analyses of composite and grab water samples. 


\section{SECTION 3 - SUMMARY AND CONCLUSIONS}

\section{A. FLOW DATA SUMMARY}

1. Flow data was gathered at all five sites over a 24-hour period (approximately 2 p.m., October 5 th through 2 p.m., October 6th, 1983). Only $0.01^{\prime \prime}$ rainfall had been experienced in the area for 14 days previous to the testing and $0.02^{\prime \prime}$ was recorded at 9:30 p.m. October 5, 1983. Instantaneous and continuous flow readings were obtained at each site using direct depth measurements and ISCO Model 1870 flow meters. Due to technical problems with some of the flow meters, the data recorded continuously on charts was not considered accurate for sites 1, 2, 3, and 5. Therefore, only the manually obtained depths and corresponding flow estimates were considered reliable throughout the 24-hour period.

2. The 7 Q 10 (the lowest 7 day average flow expected on an average of once in 10 years) for the USGS gaging station located about 2 miles downstream of Site 4 was calculated to be 1.1 CFS. Based on the ratio of contributing drainage areas, the calculated $7 \quad 0 \quad 10$ at Steed's Pond (Site 4) was estimated to be 0.88 CFS (395 gpm).

3. Actual flow measurements at Site 4 yielded only 0.43 CFS (193 gpm) and therefore the estimate of the 7 Q 10 based on the USGS gaging 
station information is not credible. Soil samples taken by Mr. Virgil Rogers of the Soil Conservation Service on October 19, 1983 indicated a high degree of permeability $\left(25^{\prime \prime} / \mathrm{hr}\right)$ in the area downstream of Steed's Pond earthen dam. Rough estimates show that a flow rate of several hundred gallons per minute could be passing through the earthen dam and ground immediately beneath Steed's Pond. Under dry weather conditions, only the flow escaping through the cracks in the wooden section of the dam was observed to contribute surface water to the downstream Tim's Branch creek bed.

B. ANALYTICAL DATA SUMMARY

1. Section 6 provides the results of the analytical tests performed on the samples with the exception of TTO (total toxic organics), which are still in progress.

2. The analytical information available to date indicates no contamination of Tim's Branch from sources identified as Site 2 and Site 3. A relatively high iron content $(7.0 \mathrm{mg} / 1)$ was noted in the sample from site 5 (referred to subsequently as "No Name Pond"). Further investigations are recommended to establish the reason for high iron from this source. 
C. CONCLUSION

No reliable estimate of the 7 Q 10 at Site 4 can be obtained by conventional means since the measured flow at site 4 was significantly less than the flow estimated by proportioning the downstream USGS 7 Q 10 flow based on respective drainage areas. A flow rate slightly lower than that measured ( $0.40 \mathrm{CFS})$ is recommended at this time for the 7 Q 10. Should this prove not to be acceptable, a more detailed hydrological study will be required to determine the 7 Q 10 at site 4. 


\section{SECTION 4 - DESCRIPTION OF STUDY AREA}

A. DRAINAGE AREA DESCRIPTION

According to page 230 of the 1981 Water Resources Data, South Carolina, Water Year 1981, the Tim's Branch drainage basin consists of 17.5 square miles of surface area (from "on-plant" and "off-plant" areas draining into the existing U.S.G.S. gaging station at Tim's Branch downstream of Steed's Pond (refer to Appendix K). The land is largely wooded (softwood) and rolling with some marshy areas and includes developed portions of the "300" and "700" Areas. The soil is generally clayey sand with varying amounts of clay and sand. The Tim's Branch is a tributary of the Upper Three Runs Creek which in turn is a tributary of the Savannah River. A map outlining the drainage area within the SRP is shown in Appendix A.

Five sites were selected for making flow and analytical measurements. The locations of these sites are shown in Appendix $B$. A description of these sites is as follows:

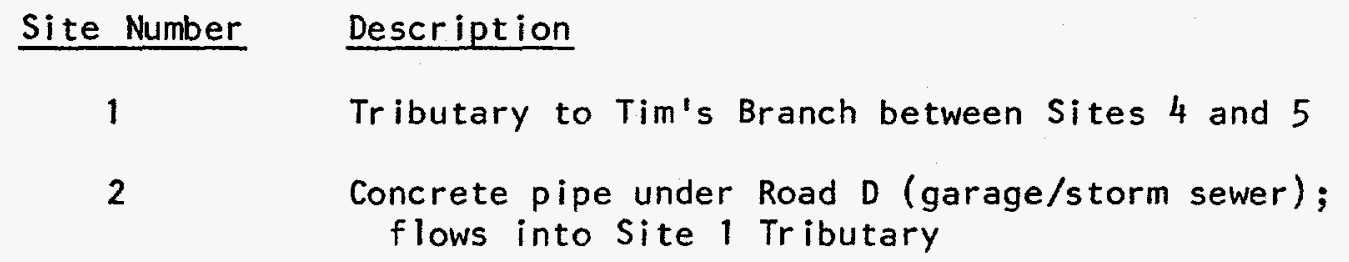


Existing weir at cooling water outfall; flows into Site 1 Tributary

"No Name" pond, downstream from twin culverts; headwaters of Tim's Branch

The highest point in the drainage area that remains perennially wet is estimated to be approximately $1 / 2 \mathrm{mile}$ upstream from Site 5 where an east-west high voltage power line crosses the stream bed.

The known man-made flow contributors to Tim's Branch include the flows as measured at Sites 2 and 3.

\section{B. STREAM CLASSIFICATION}

One criteria for determining the allowable waste load for a stream is its classification. Stream classifications are issued by the South Carolina Department of Health and Environmental Control for those surface bodies of water in South Carolina. A stream classification is based upon hydrological study of a drainage basin to determine the existing biota which rely on the water resources within the basin, to determine the water resources, to quantify these sources, and to determine their downstream uses. The purpose of a stream classification is to ensure that a stream's natural conditions are preserved as well as to protect the users of the water resources. 
Tim's Branch does not have a listed classification. The Savannah River, however, is classified as Class B for the reach between Clark Hill Reservoir and Fort Pulaski. Tim's Branch is tributary to this reach via Upper Three Runs Creek. Thus, it is assumed by the South Carolina Department of Health and Environmental Control that Tim's Branch would also be a Class B stream. As enacted by the State of South Carolina, a Class B stream is defined as follows:

\begin{abstract}
"Class B - freshwater suitable for secondary contact recreation and as a source of drinking water supply after conventional treatment in accordance with requirements of the Department. Suitable for fishing, survival and propagation of fish, and other fauna and flora. Suitable also for industrial and agricultural uses."
\end{abstract}

The specific standards for a class B stream and general stream classification criteria are presented in Appendix C. 


\section{SECTION 5 - STREAM FLOW AND ANALYTICAL RESULTS}

\section{A. FLOW DATA}

During the week preceding the 24 hour flow measurements, 90 degree $V$-notch weirs were constructed at sites $1,2,4$ and 5 . It was concluded from preliminary site investigations that the $V$-notch weirs would be adequate to measure the flows which were all estimated to be less than 2 CFS. The existing rectangular weir at site 3 was capable of measuring a wide range of flows and was also acceptable. The design sketches used to fabricate dams and weirs are included in Appendix D.

Upon completion of the dam and weir construction, LGE set up an ISCO Model 1870 flow meter, which continuously records depth, and an ISCO Model 1580 automatic sampler at each site. In order to verify the instrument readings, direct reading measurements were made at 4 hour intervals using rulers. During the course of the 24-hour monitoring period, technical problems with the equipment (erroneous chart readings and weak batteries) made it necessary to rely on the manual measurements. A detailed record of depth measurements and corresponding calculated flows is included in Appendix E. A narrative description of the 24-hour flow testing is included in Appendix F. 
The flow test was preceded by 14 days of no significant rain according to rainfall records (refer to Appendix G). The results of the 24-hour dry weather flow measurements are summarized below:

\begin{tabular}{|c|c|c|c|c|}
\hline Site No. & \multicolumn{2}{|c|}{ Average Flow } & \multicolumn{2}{|c|}{ Range of Flow Over 24 Hours } \\
\hline & (CFS) & (GPM) & (CFS) & (GPM) \\
\hline 1 & 0.525 & 236 & $0.490-0.592$ & $220-266$ \\
\hline 2 & 0.138 & 62 & $0.125-0.160$ & $56-72$ \\
\hline 3 & 0.637 & 286 & $0.615-0.692$ & $276-311$ \\
\hline $4 *$ & 0.403 & 181 & $0.397-0.420$ & $178-189$ \\
\hline 5 & 0.420 & 189 & 0.420 & 189 \\
\hline
\end{tabular}

*Does not include an estimated 15 GPM flow observed from a small tributary downstream of we ir.

The flow measurement results lead to several observations as follows:

1. The flow at Site 1 was significantly less than the sum of flow at Sites 2 and 3 . No known surface or piped flow is known to enter or leave the tributary between these two locations. The known flows from sites 2 and 3 into the tributary total 0.775 CFS; however, the flow through the weir at Site 1 was 0.525 CFS. The difference of 0.25 CFS could be due to exfiltrate from the stream into the ground through porous native soils underlying the tributary bed. 
The dam at Site 1 created a water level with a $20^{\prime}$ to $30^{\prime}$ long perimeter, such that complete water tightness along the length of the dam was not achieved. Some water losses bypassing the weir were noticed, but not considered significant since the flow losses observed were trickles of water estimated to be on the order of 2 to 3 GPM $(0.004$ to 0.007 CFS).

2. The flow measured at Site 4 was significantly less than the sum of flows from Sites 1 and 5. The known average flows into Steed's Pond (Sites 1 and 5) total $0.945 \mathrm{CFS}$, compared to the Site 4 flow measurement of 0.403 CFS. A minor stream was noted entering Tim's Branch about 100 ' downstream from Site 4. The stream could possibly be the result of seepage through or under the Steed's Pond earthen embankment dam. The surface flow of the minor stream was estimated to be on the order of $15 \mathrm{gpm}(0.033 \mathrm{CFS})$ bringing the total estimated outflow from Steed's Pond to 0.436 CFS.

Since the sum of the inflows were significantly greater than the outflow, a brief investigation was made as to the soil type in the area of the pond to confirm or deny the hypothesis that the difference in flows was primarily due to flow into the ground water.

On October 19, 1983, Mr. Virgil Rogers, a soil scientist with the Soil Conservation Service, was contacted to obtain some permeability data for native soil in the area of Site 4. The results 
of this testing indicate that a permeability value on the order of 25 " per hour can be expected in the sand/gravel mixture. It was Mr. Rogers' opinion that a very large percentage of the pond's water was escaping through this permeable soil. Some rough calculations made using some simplistic assumptions verify that losses through the earthen dam could add up to hundreds of gallons per minute.

Evaporative losses from Steed's Pond were also calculated based on an annual evaporation of 44 inches. This is equivalent to an average of $0.07 \mathrm{CFS}$ or about $30 \mathrm{gpm}$, not enough to account for the 0.509 CFS difference.

3. The flow measured at site 4 is less than the drainage area proportioned flow based on low flow data at the USGS gaging station downstream from Site 4. The USGS daily flow records for Tim's Branch at Road C, are included in Appendix $H$.

The lowest daily flow on record $(3 / 74-9 / 82)$ at the Tim's Branch USGS station was 1.0 CFS. There are 3.53 additional square miles of land draining into Tim's Branch between Site 4 and the USGS Station. The drainage area ratio can then be used to estimate the flow at site 4 . This ratio is $(17.5-3.53) /(17.5)$, or $80 \%$. The resulting lowest daily flow at site 4 is calculated to be $0.8 \times 1.0 \mathrm{CFS}=0.80 \mathrm{CFS}$. This calculated flow is greater than the measured flow of 0.436 CFS. The reasons for this difference $5-4$ 
are possibly due to exfiltration from Steed's Pond as previously noted. A hydrologic investigation would be required to confirm the reason for these flow differences.

\section{B. CHEMICAL ANALYSES}

The chemical constituents selected for analysis were recommended by LGE to provide general background data on heavy metals, organics, and typical water related chemical indicators. The recommended list was approved by DuPont without modification.

Composite and grab samples were collected at each of the five sites. The samples were refrigerated and shipped off-site for analys is by independent laboratories (SCL and Mead CompuChem). A list of the constituents analyzed and their respective results are provided in detail in Appendix 1 and summarized in Section 6. Also included in Appendix $\mathrm{I}$ is the complete report prepared by Scientific Control Laboratories, Inc. Only the TTO (total toxic organics) results are not completed at this time.

The collection of samples was accomplished by two methods: composite and grab. The composite samples were collected by the ISc0 samplers which were activated by the ISCO flow meters. The samplers were set such that a sample would be collected every time a predetermined volume of water (as measured by the flow meter) flowed over the weir. The samplers and flow meters were calibrated so that a sufficient 
volume of sample for laboratory analys is would be collected over the 24-hour period of the study. Unfortunately, equipment malfunction resulting from battery failure prevented proper sample collection at Sites 1,3 and 5 . At the end of the 24-hour sampling period, grab samples were added to the water collected at these sites to ensure sufficient sample quantity for analysis. For this reason, only sites 2 and 4 represent flow proportional composite samples.

Grab samples were collected every four hours at each site for analysis of volatile total toxic organics (TTO). These samples were collected in a manner so as to not allow any air into the sample bottle. The seven grab samples for each site were then combined into one time averaged composite by SCL, Inc. 


\section{SECTION 6 - EVALUATION OF RESULTS}

A. DETERMINATION OF 7 Q 10 FOR TIM'S BRANCH AT SITE 4

"7 Q $10^{\prime \prime}$ is a short hand abbreviation for the lowest expected 7 day average daily flow in an average 10 year period. It is presumed that this statistic would be used in determining a practical low flow for permissible wastewater loading.

This flow was to be determined at Site 4 since this is the point considered representative of dilution water flow upstream of a future waste treatment plant outfall.

The procedure used to determine the Tim's Branch USGS 7 Q 10 was obtained by the method by H. C. Riggs in Chapter B-1, "Low Flow Investigations", of Techniques of Water Resources Investigations of the United States Geological Survey. The data and graph that was used to calculate the 7 Q 10 is included in Appendix J. Although some deviation does occur and the period of study is relatively short ( 9 years), a fairly definitive straight line is able to be drawn to establish a pattern. On the basis of this data, a stream flow of 1.1 CFS is estimated to be the 7 Q 10 at the Tim's Branch USGS gaging station. 
Since USGS flow data at Site 4 does not exist, the only readily available method of estimating the 7 Q 10 at site 4 is to proportion the flow based on the ratio of drainage areas; or another method is to use the ratio of simultaneous low flow measurements at site 4 and the USGS Station.

The drainage area at the USGS station is listed in the USGS Water Data Report SC-81-1 as 17.5 square miles (refer to Appendix K). The area drained by Tim's Branch between Site 4 and the USGS gaging station is shown in Appendix $A$ and measures 3.53 square miles. Site 4 therefore drains $(17.5-3.53) \div 17.5=80 \%$ of the area draining to the USGS station. Therefore, $80 \%$ of $1.1 \mathrm{CFS}=0.88 \mathrm{CFS}$, which is the 7 Q 10, calculated by proportioning drainage areas.

Simultaneous low flow measurements (at the USGS station and site 4) were not available since the USGS station is no longer operating, according to DuPont personnel who investigated the possibility of obtaining this information. Therefore, the method of calculating 7 Q 10 by the ratio of simultaneous low flow measurements could not be used.

Since the measured flow totaled 0.436 CFS (including the 0.033 CFS tributary downstream from Site 4), the proportional drainage area method results in a 7010 flow approximately twice as high as the measured daily flow. It should be noted that the measured daily flow is based on one 24-hour flow measurement, while the 7 Q 10 is 
projected on a 7 day average flow basis. However, historical data (Appendix H) indicate that the 7 Q 10 at the USGS station (1.1 CFS) is only $10 \%$ higher than the lowest daily flow $(1.0$ CFS) recorded at the USGS station.

According to conversations with the South Carolina Department of Health and Environmental Control, a parameter sometimes associated with 7 Q 10 flows in that area of the state is 0.18 CFS per square mi le of drainage area. Since 13.97 square mi les drains into the Site 4 area, a "rule of thumb" 7 Q 10 of 2.5 CFS is obtained, obviously too high for use at site 4 in view of the much lower observed flow.

Due to the limited data available, a flow somewhat less than the measured flow is recommended for estimating the low flow dilution available from site 4 . A low flow of 0.40 CFS (180 GPM) at site 4 is recommended for low flow dilution estimates and should be acceptable for regulatory permitting purposes since flow measurements were taken during dry weather conditions, prior to which no significant rainfall had occurred over a period of 14 days.

\section{B. ANALYTICAL RESULTS}

Table 6-1 summarizes the analytical results. The asterisks indicate those tests which could not be performed on the samples due to an insufficient volume. The cause of the sample loss is unknown. 
However, it is suspected that the losses were a result of spillage during shipping of the samples to the laboratory.

As illustrated by Table 6-1, constituent levels in general appear to be relatively low and of the same magnitude expected from natural surface water sources. It is curious, however, that iron is evident in substantially higher quantities at sites 4 and 5 in comparison with the other sites. The source of the elevated iron concentration may be located upstream of Site 5 , or may be naturally occurring in the supply of water to Site 5. However, further site study would be required to make any speculations regarding its source. 
TABLE 6-1

ANALYTICAL RESULTS

CONCENTRATIONS (MG/L)

PARAMETER

SITE 1 SITE 2 SITE 3 SITE 4 SITE 5

Sodium

0.4

0.3

1.0

0.9

0.6

Copper

0.01

0.02

0.04

$<0.01$

$<0.01$

Lead

$<0.01$

$<0.01$

$<0.01$

$<0.01$

$<0.01$

Nickel

$<0.01$

$<0.01$

$<0.01$

0.0

$<0.01$

Iron

Total Phosphorus

1.0

0.11

0.4

5.2

7.0

0.4

0.3

0.2

0.3

0.4

0il \& Grease

0.6

0.2

*

0.5

*

$\mathrm{pH}$

Uranium

Total Dissolved Solids

Total Organic Carbon

Aluminum

Nitrate

7.45

7.40

7.40

7.20

6.04

$<2.0$

$<$

2.0

$<2.0$

$<2.0$

$<2.0$

61.0

69.0

$\star$

60.0

$*$

10.0

9.0

7.0

20.0

10.0

0.6

0.1

$<0.0$

$<0.01$

$<0.1$

0.32

0.29

4.0

3.3

3.2

Total Suspended Solids

2.0

4.0

$\star$

2.0

$*$

Arsenic

$<0.001<0.001$

$<0.001$

$<0.001$

$<0.001$

Nitrite

Boron

$<0.1$

$<0.1$

$\star$

$<0.1$

$<0.1$

0.1

0.1

0.4

1.3

$<0.1$

Cadmium

$<0.01$

$<0.01$

$<0.01$

$<0.01$

$<0.01$

Chromium

0.01

0.01

0.01

0.01

0.01

Lithium

$<0.01$

$<0.01$

$<0.01$

$<0.0$

$<0.01$

Phenol

Sulfate

Chloride

Barium

$<0.1<0.1$

$*$

$*$

$*$

3.0

2.0

$*$

7.0

3.5

3.5

3.2

$*$

3.3

1.1

$<0.01<0.01$

$<0.01$

$<0.01$

$<0.01$

Mercury

$<0.0001<$

$<0.0001<0.0001<$

$0.0001<$

$<0.001<0.001$

$<0.001$

$<$

$0.001<0.001$

Silver

$<0.01$

$<.0 .01$

$<0.01$

$<0.01<0.01$

TTO *\%

*Insufficient Volume of Sample

**Analytical testing in progress 


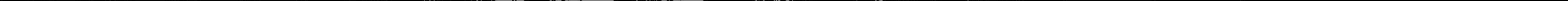




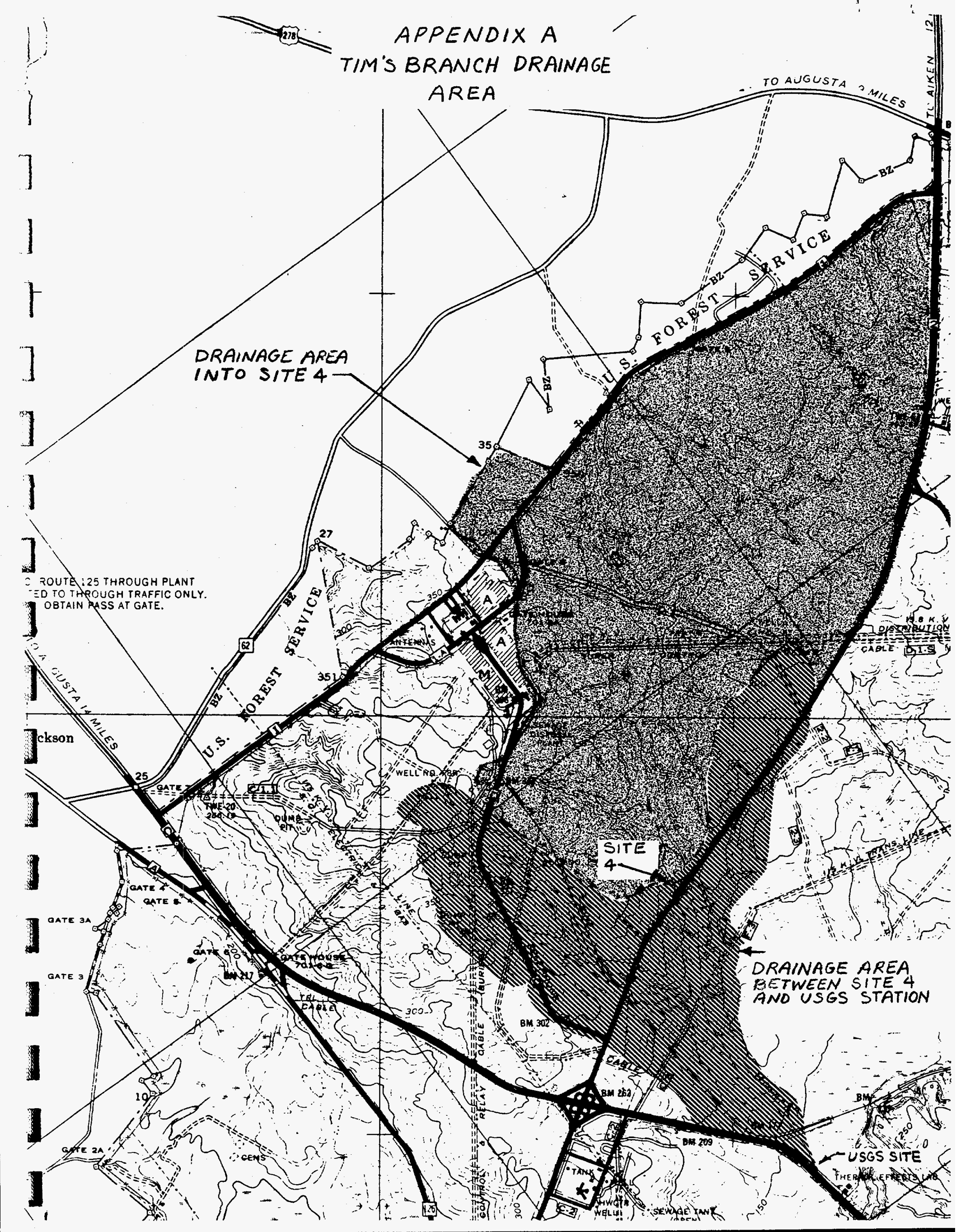




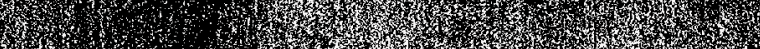




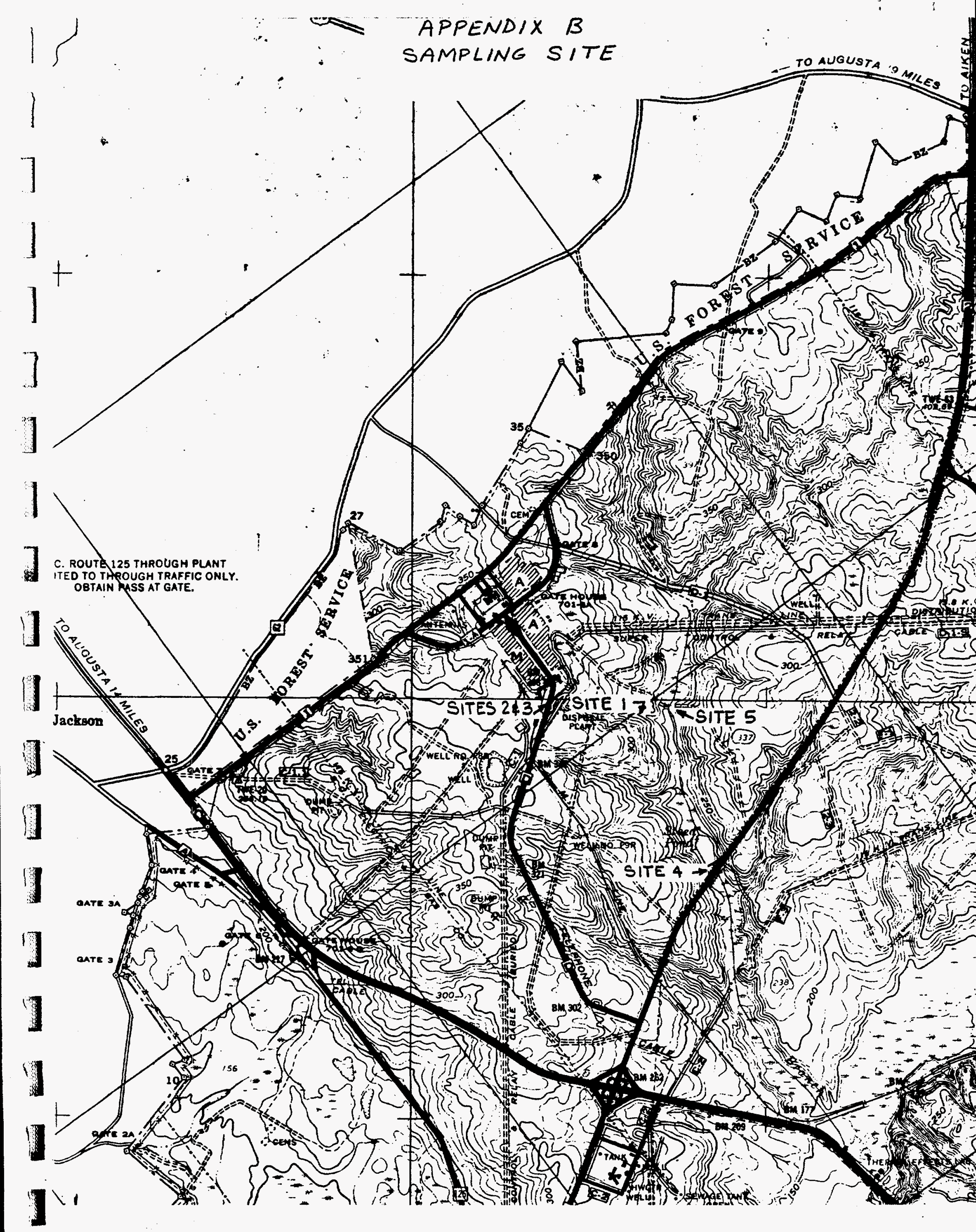




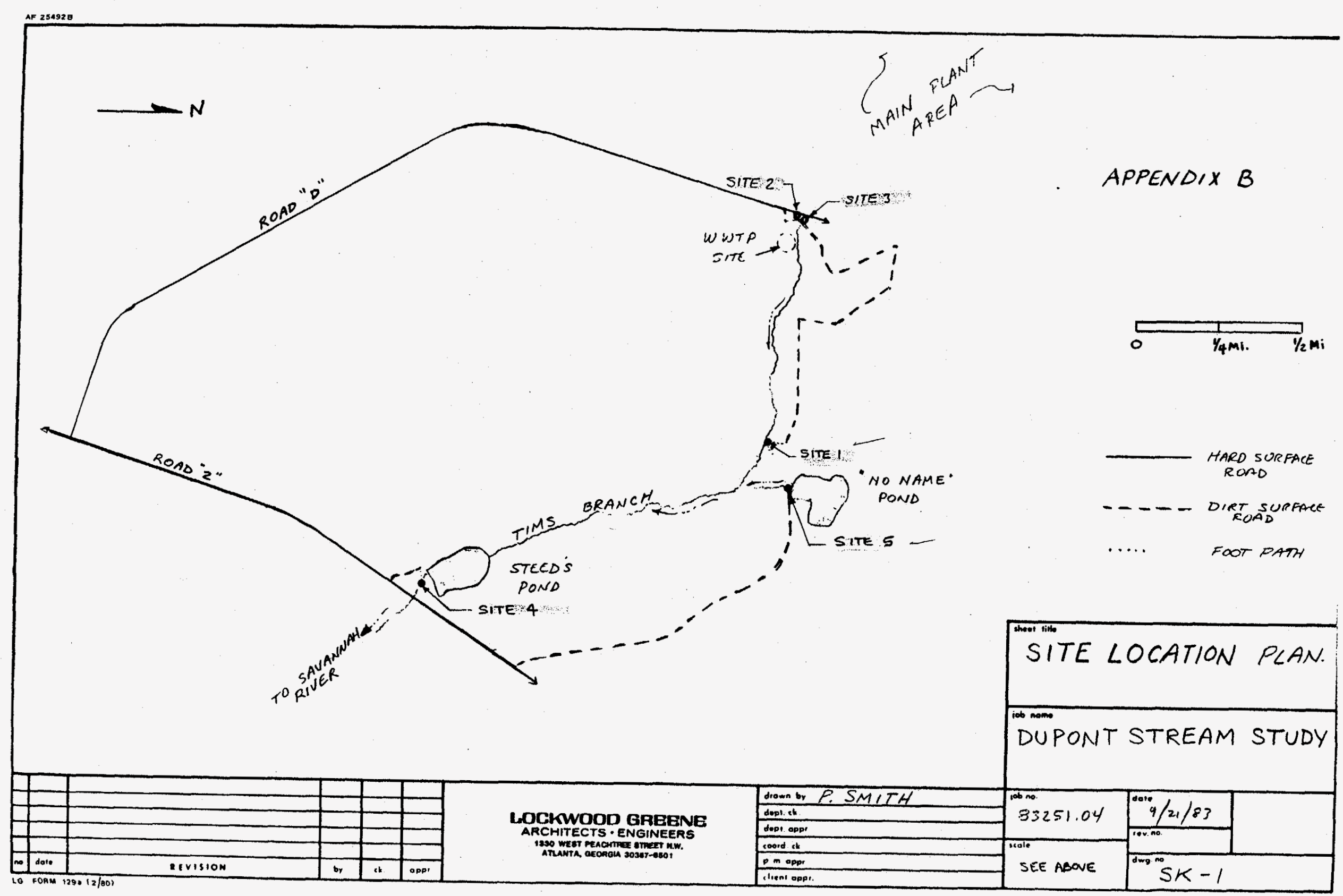




\section{APPEND IX C - SOUTH CAROL INA WATER CLASS IFICATI ON STANDARDS}

1.

7

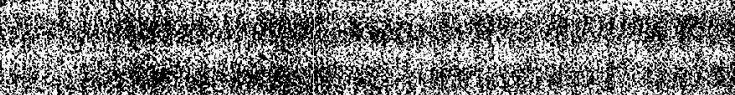
W

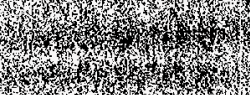
removed

]

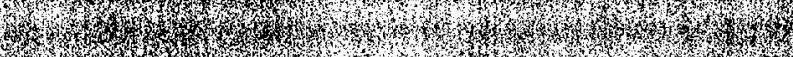

1.

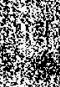

7

]

1.1,

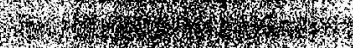

\%

1.

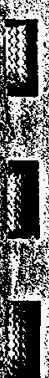

X.

7.

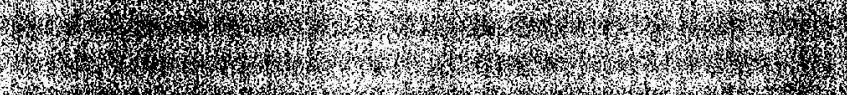

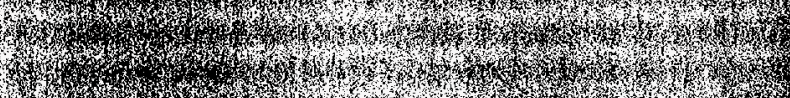

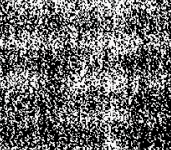

1.

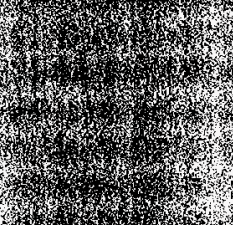

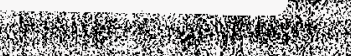

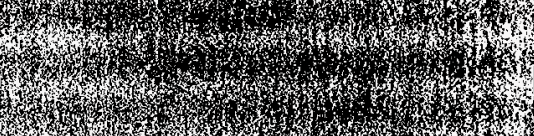

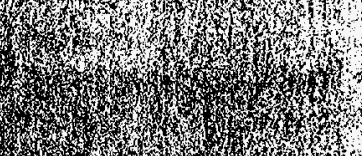




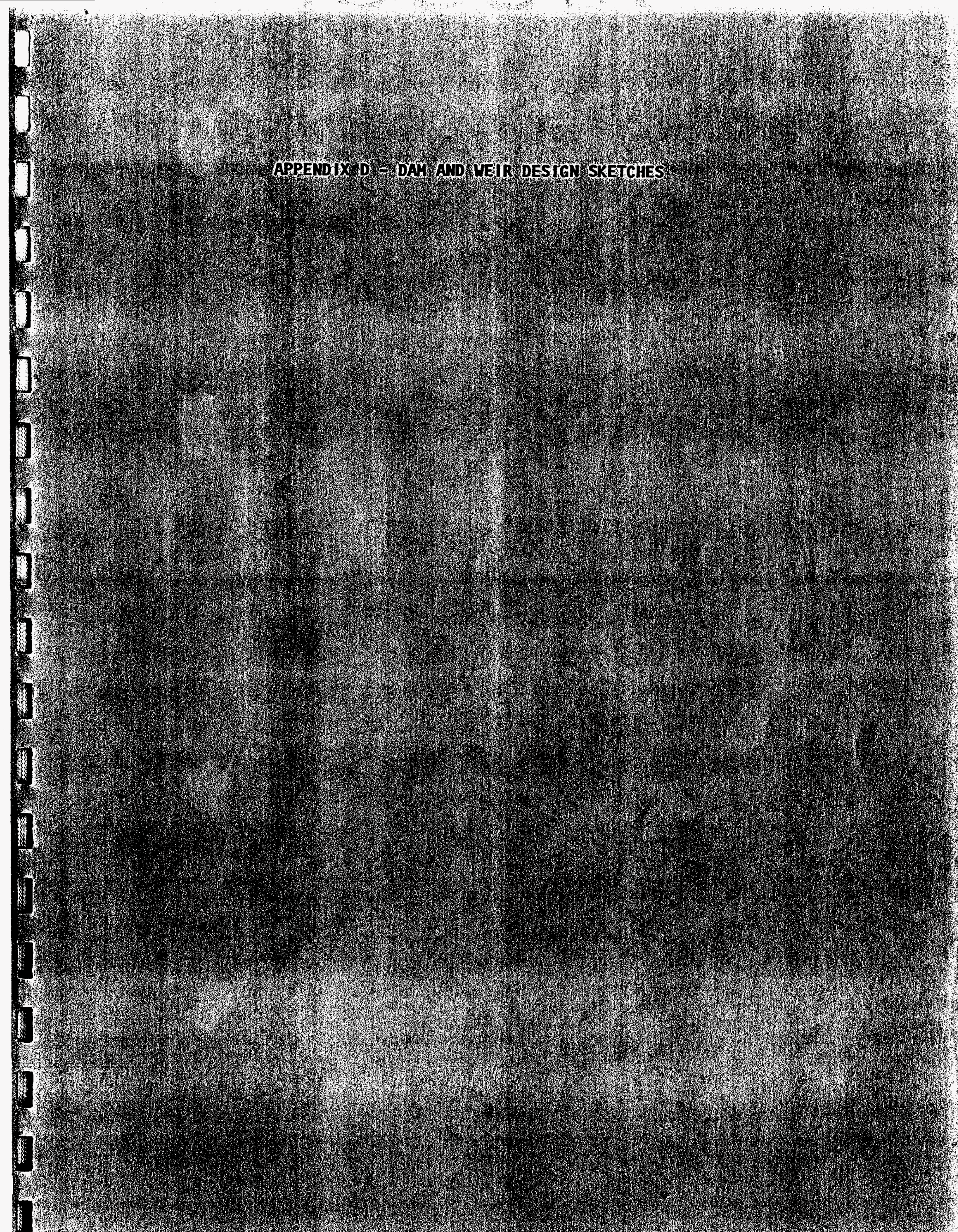

APPEND X D NDAM AND WER DESTG SKETCHES 


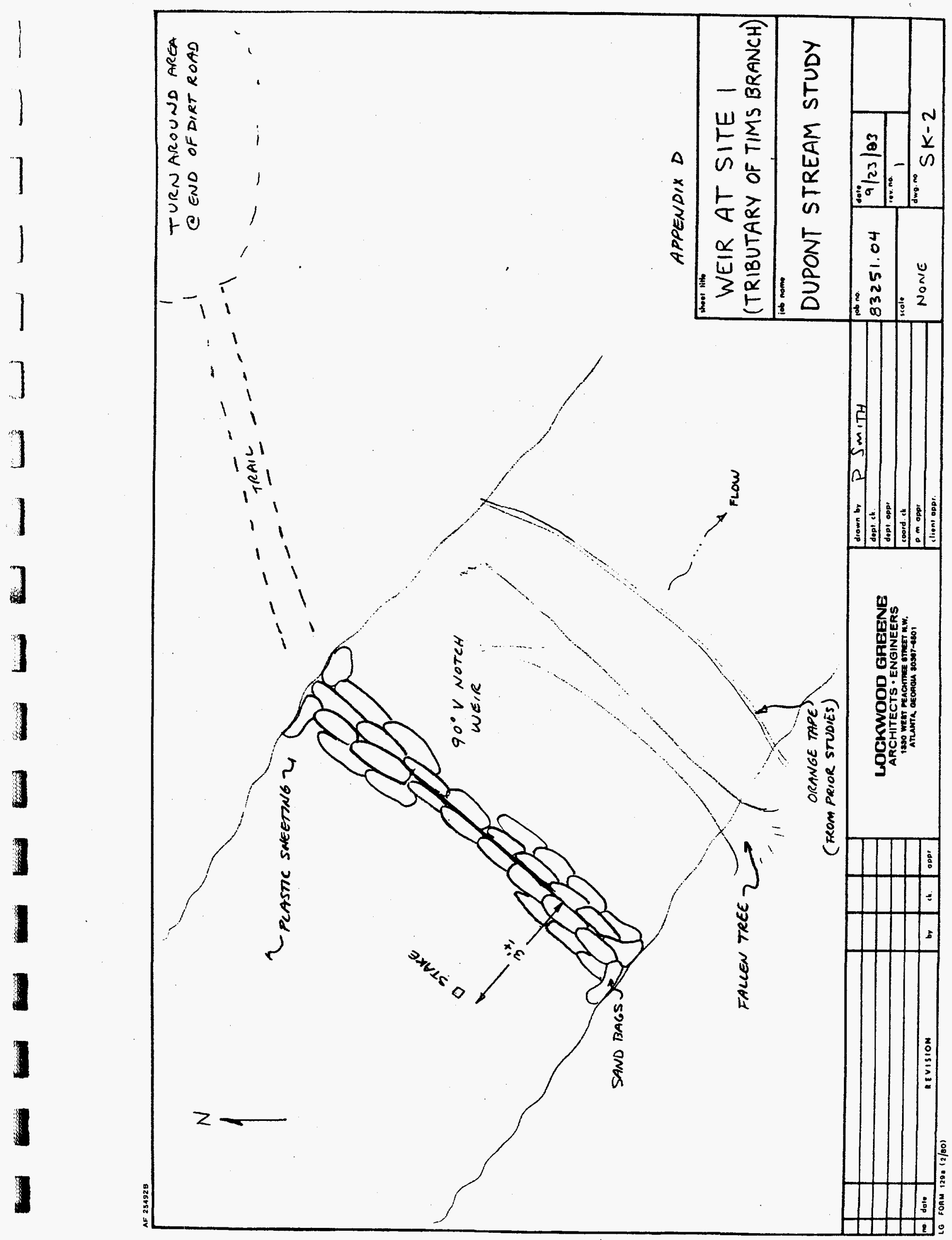




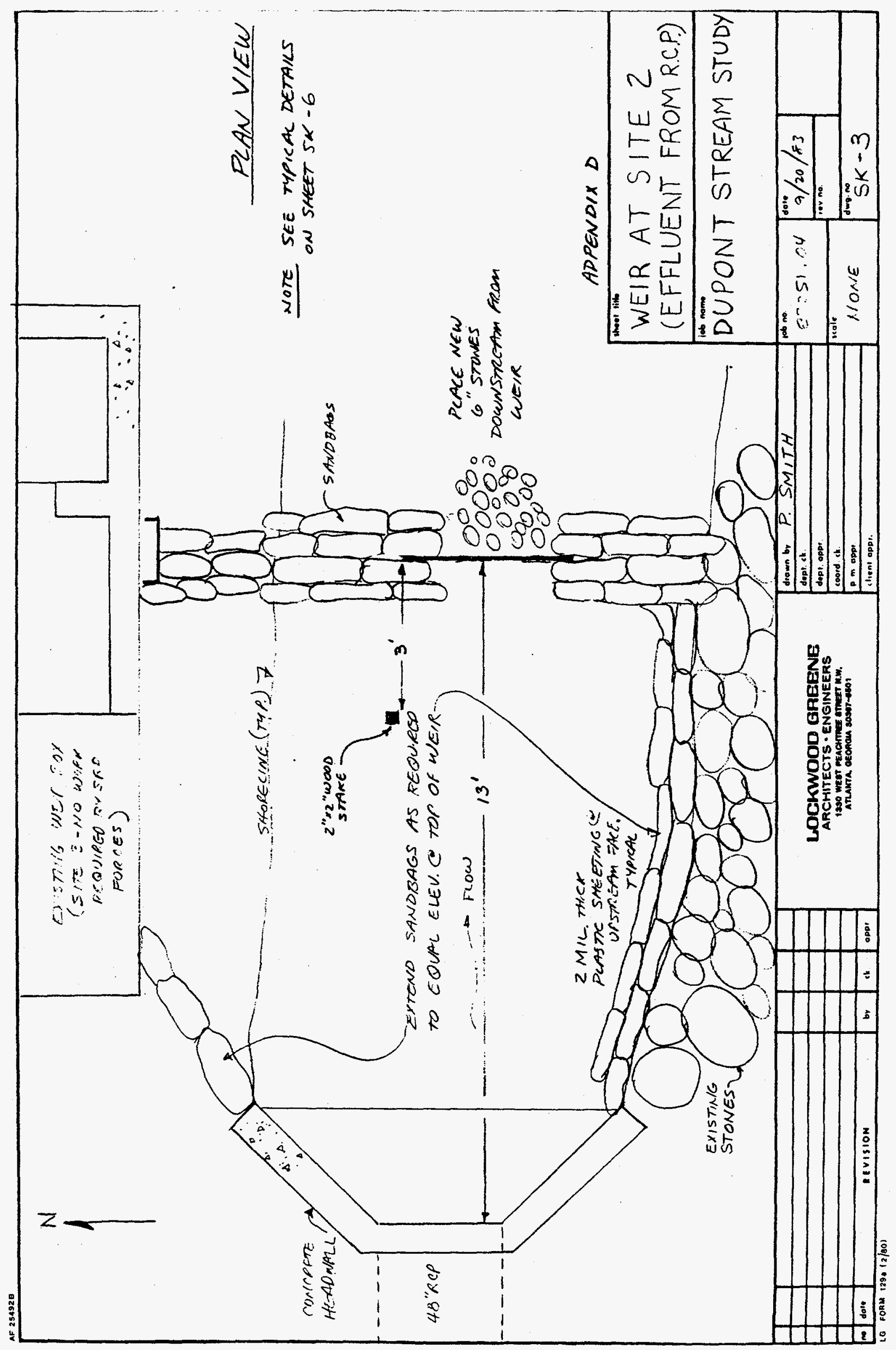




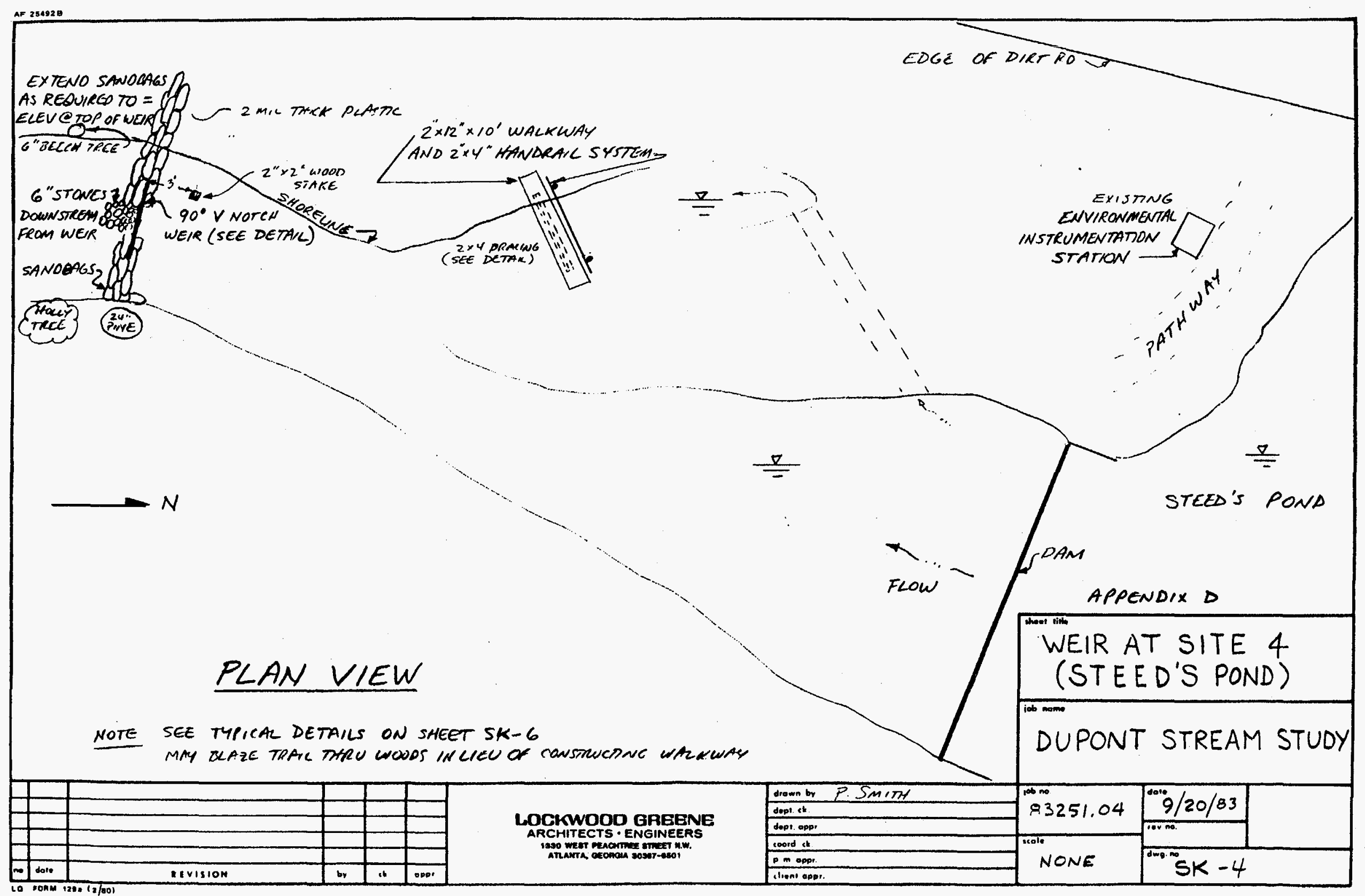




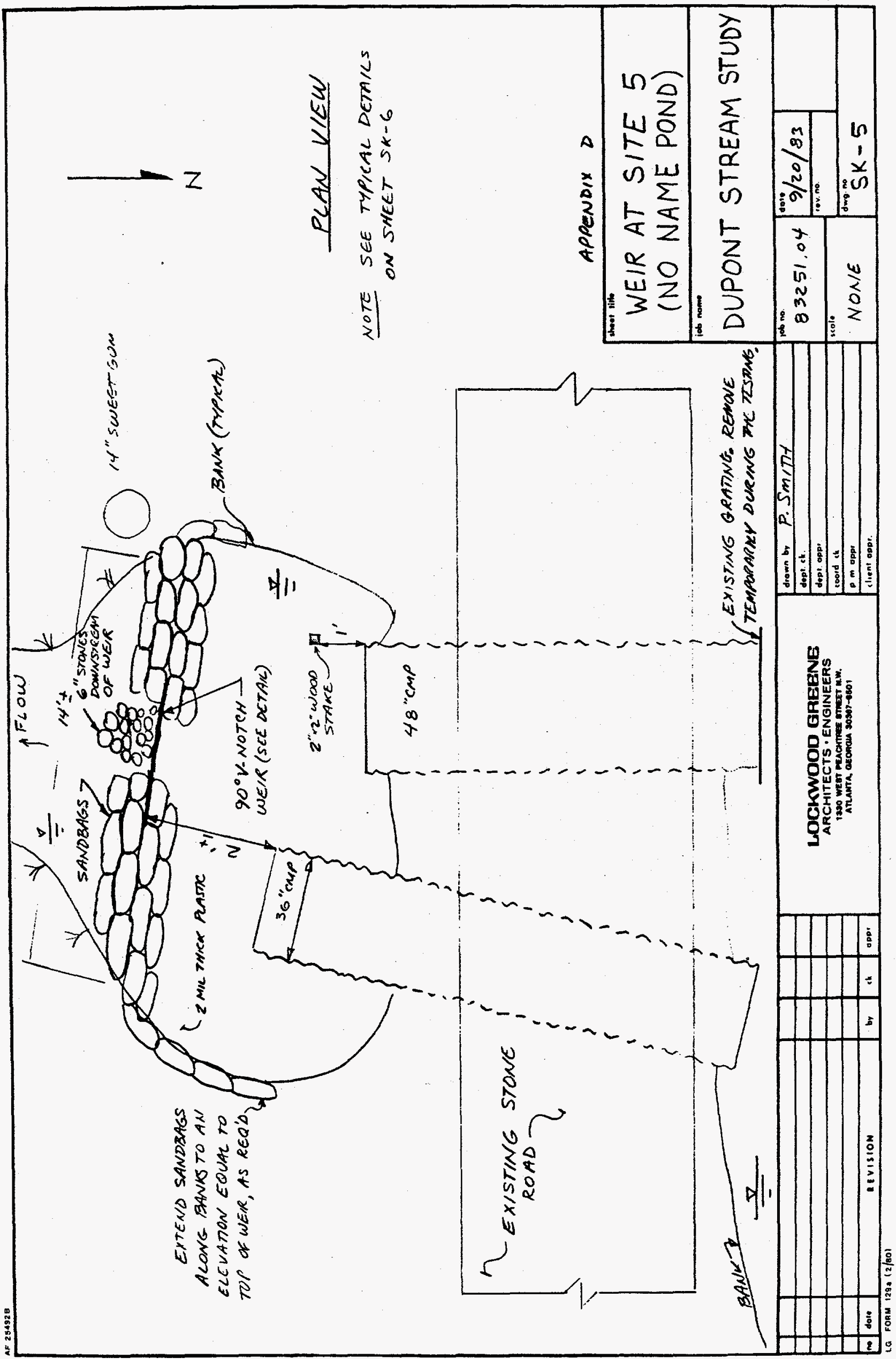




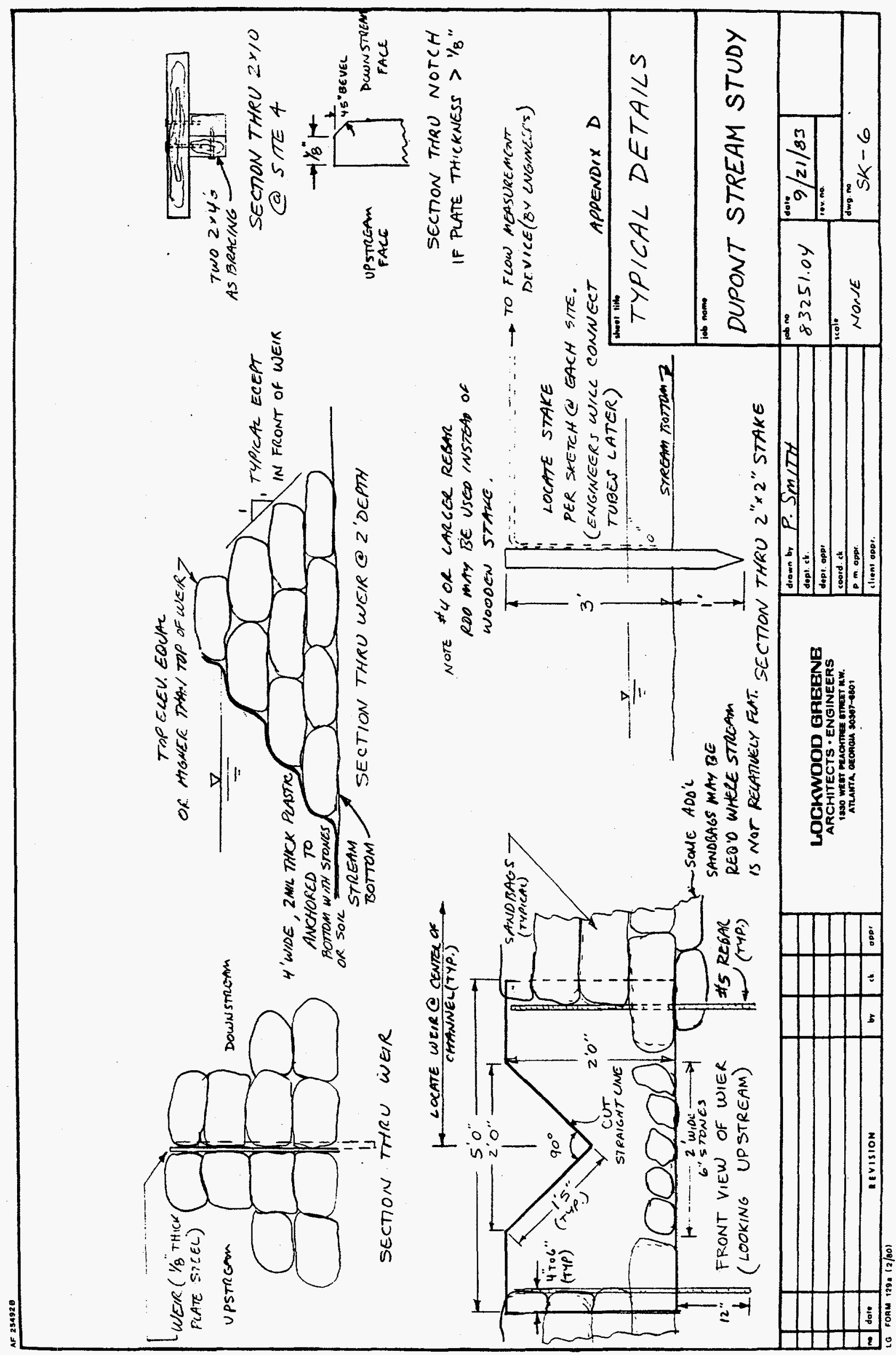


(3)

.

APPENDIX E T TLOW MEASUREMENT DATA

1

I)

1

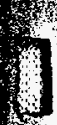

xit

7.

3.



(3)

(6)





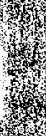

\section{(3)}

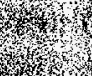

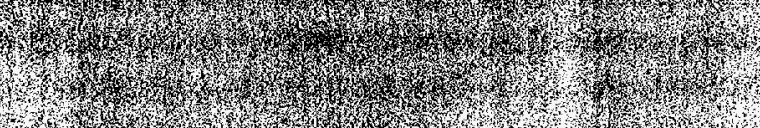
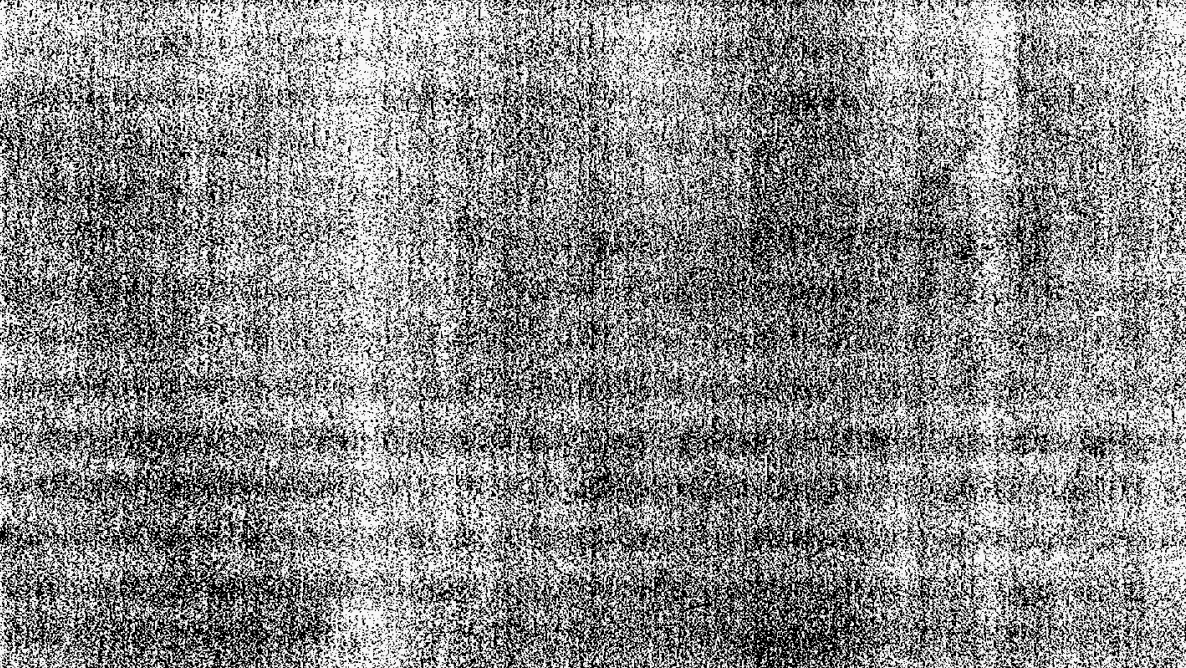

3.

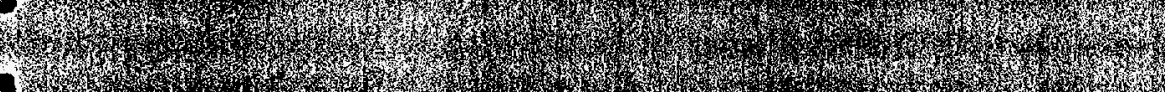

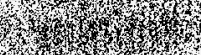

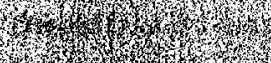




\section{LOCKWOOD GREENE \\ ARCHITECTB - ENGINEERE \\ ATLANTA}

APPENDIY E

JOBNO. 83251.04

SHEET NO. 1 of $z$

DATE $10 / 6 / 83$

JOBNAME DUPONT

SUBJECT SITE $* 1, * 2, \# 3, * 4, * 5$

COMPUTED BY BC

CHECKED BY PIS

\begin{tabular}{|c|c|c|c|c|c|c|c|}
\hline & APiat & TIME & $\begin{array}{c}0 \\
\text { MEASUEDD } \\
\text { DEPTI }\end{array}$ & $\begin{array}{c}\text { Q } \\
\text { METERED } \\
\text { DEPTH }\end{array}$ & 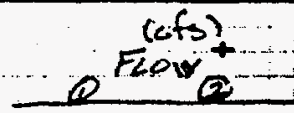 & $\begin{array}{l}\text { METERES } \\
\text { JoTAt: } \\
\text { YeLi }\end{array}$ & $\begin{array}{l}\text { Tor } y=4 \\
\text { ANG } \\
\text { FLE }\end{array}$ \\
\hline \pm 1 & 1 & 1355 & $0.541 \mathrm{ft}$ & $0.548 \mathrm{ft}$ & 0.538 & --- & $-\quad-\cdots$ \\
\hline & 2 & 1755 & $0.521 \mathrm{ft}$ & $0.527 \mathrm{ft}$ & 0.504 & 7660 ef & $0.532 .0 f s$ \\
\hline & 3 & 2210 & $0.541 \mathrm{ft}$ & $0.540 \mathrm{ft}$ & 0.538 & $15370 \mathrm{ef}$ & $0.518 \mathrm{cfs}$ \\
\hline & 4 & 0205 & $0.521 \mathrm{ft}$ & $0.526 \mathrm{ft}$ & 0.502 & $23330<f$ & 0.533 efs. \\
\hline & 5 & 0550 & $0.521 \mathrm{ft}$ & $0.534 \mathrm{ft}$ & 0.490 & $34430 \quad<F$ & $0.601 \mathrm{cfs}$ \\
\hline & 6 & 0940 & $0.541 \mathrm{ft}$ & $0.550 \mathrm{ft}$ & 0.538 & 51150 cf & $0.7 i 9 . f S$ \\
\hline & 7 & .1345 & $0.562 \mathrm{ft}$ & $0.573 \mathrm{ft}$ & 0.621 & $69220 \mathrm{cf}$ & $0.807<f s$ \\
\hline$\# 2$ & 1 & 1420 & $0.312 \mathrm{ft}$ & $0.310 \mathrm{ft}$ & 0.136 & $\cdots-$ & $-\quad-\ldots$ \\
\hline & 2 & 1820 & $0.312 \mathrm{ft}$ & $0.310 \mathrm{ft}$ & 0.136 & 1420 \&f & $0.099 \mathrm{cfs}$ \\
\hline & 3 & 2225 & $0.302 \mathrm{ft}$ & $0.315 \mathrm{ft}$ & 0.125 & 3460 cf & $0.119 \mathrm{cfs}$ \\
\hline & 4 & 0235 & $0.323 \mathrm{ft}$ & $0.333 \mathrm{ft}$ & $0.160^{\circ}$ & $5730^{\circ}$ if & $0.130<f s$ \\
\hline & 5 & 0610 & $0.332 \mathrm{ft}$ & $0.337 \mathrm{ft}$ & 0.160 & 7910 \&f & 0.139 efs. \\
\hline & 6 & 1000 & $0.312 \mathrm{ft}$ & $0.337 \mathrm{ft}$ & 0.165 & 10170 c & $0.144 \mathrm{crs}$ \\
\hline & 7 & 1420 & $0.302 \mathrm{ft}$ & $0.301 \mathrm{ft}$ & 0.124 & $123604 \mathrm{f}$ & $0.143 \mathrm{c} s \mathrm{~s}$ \\
\hline & +1 & 1425 & $0.240 \mathrm{ft}$ & $0.238 \mathrm{ft}$ & 0.608 & $=$ & $-\cdots$ \\
\hline & 2 & 1825 & $0.240 \mathrm{ft}$ & $0.238 \mathrm{ft}$ & 0.608 & 9130 of & $0.634 \mathrm{cfs}$ \\
\hline & 3 & 2225 & $0.250 \mathrm{ft}$ & $0.250 \mathrm{ft}$ & 0.654 & 16570 of & $0.575 \mathrm{efs}$ \\
\hline$*$ & 4 & 0230 & $0.240 \mathrm{ft}$ & - & 0.615 & 25510 ef & 0.586 efs \\
\hline & 5 & 0607 & $0.240 \mathrm{ft}$ & - & 0.615 & 27450 ef & $0.486 . \mathrm{fs}$ \\
\hline & 6. & 0955 & $0.250 \mathrm{ft}$ & - & 0.654 & $=\ldots \ldots$ & - \\
\hline & 7 & 1415 & $0.200 \mathrm{ft}$ & - & 0.692 & $\cdots-\cdots$ & - \\
\hline$\$ 4$ & 1 & 1445 & $0.479 \mathrm{ft}$ & $0.480 \mathrm{ft}$ & 0.399 & $=$ & - \\
\hline & 2 & 1905 & $0.479 \mathrm{ft}$ & $0.480 \mathrm{ft}$ & 0.399 & $.7630 . \mathrm{f}$ & $0.489 \mathrm{cfs}$ \\
\hline & 3 & 2245 & $0.479 \mathrm{ft}$ & $0.480 \mathrm{ft}$ & 0.397 & 12950 cf & 0.450 efs \\
\hline & 4 & 0255 & $0.490 \mathrm{ft}$ & $0.485 \mathrm{ft}$ & $0.710^{\circ}$ & 18970 if & $0.432 \mathrm{cfs}$ \\
\hline & 5 & 0625 & $0.490 \mathrm{ft}$ & $0.485 \mathrm{ft}$ & 0.410 & 24210 .cf & $0.429<\mathrm{fs}$ \\
\hline & 6 & 1010 & $0.479 \mathrm{ft}$ & $0.484 \mathrm{ft}$ & 0.407 & 29720 ef & $0.425 \mathrm{cts}$ \\
\hline & 7 & 1440 & $0.479 \mathrm{ft}$ & $0.483 \mathrm{ft}$ & 0.407 & $\quad 36450^{\circ}<f$ & $0.423 \mathrm{cfs}$ \\
\hline$\$ 5$ & 1. & 1455 & $0.490 \mathrm{ft}$ & $0.490 \mathrm{ft}$ & 0.420 & - & $\cdots-\cdots$ \\
\hline & 2 & 1920 & $0.490 \mathrm{ft}$ & $0.483 \mathrm{ft}$ & 0.420 & $\therefore 6580.6$ & 0.414 cts \\
\hline & 3 & 2255 & $0.490 \mathrm{ft}$ & $0.483 \mathrm{ft}$ & 0.420 & 11890 cf & 0.413 efs \\
\hline & 4. & 0310 & $0.490 \mathrm{ft}$ & - & $0.420 \quad-\ldots$ & 175.90 if & $0.399=4$ \\
\hline & 5 & 0640 & $0.490 \mathrm{ft}$ & - & 0.420 & $17830<f$ & 0.314 efs. \\
\hline & 6 & 1025 & $0.490 \mathrm{ft}$. & - & 0.420 & $\therefore \div$ & - \\
\hline & 7 & 1500 & $0.490 \mathrm{ft}$ & - & $0.420=$ & -1 & - \\
\hline
\end{tabular}

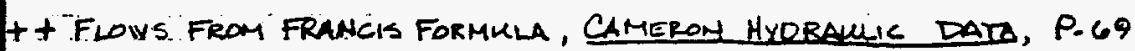

* Equipment Failure

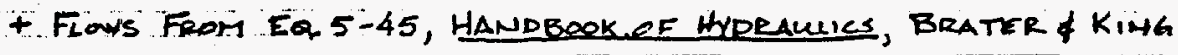


LOCKWOOD GREENE

ARCHITECTS - ENGINEERS

ATLANTA

JOBNAME DUPONT

SUBJECT_SITES \#1,2,3,4,5

Ave. Eleivs (efs)

SITE MANUAL MEAL 0.525

0.543

o. 138

2

0.637

3

0.403

5

0.420

0.146

*

0.404

$¥$

TOTALIZER

0.807 (NOT RELIAELE)

0.143

*

0.423

$*$

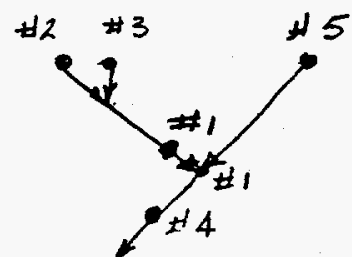

SCHEMATIC OF SITES

FLON EquATIONS:

$$
\begin{aligned}
& 90^{\circ} V \text {-NOTCH VEIR } Q=2.5 \mathrm{H}^{2.5} \\
& \text { RECT. WEIR W/END CONTR. } Q=3.33(1.620-0.2 \mathrm{H}) \mathrm{H}^{1.5} .
\end{aligned}
$$




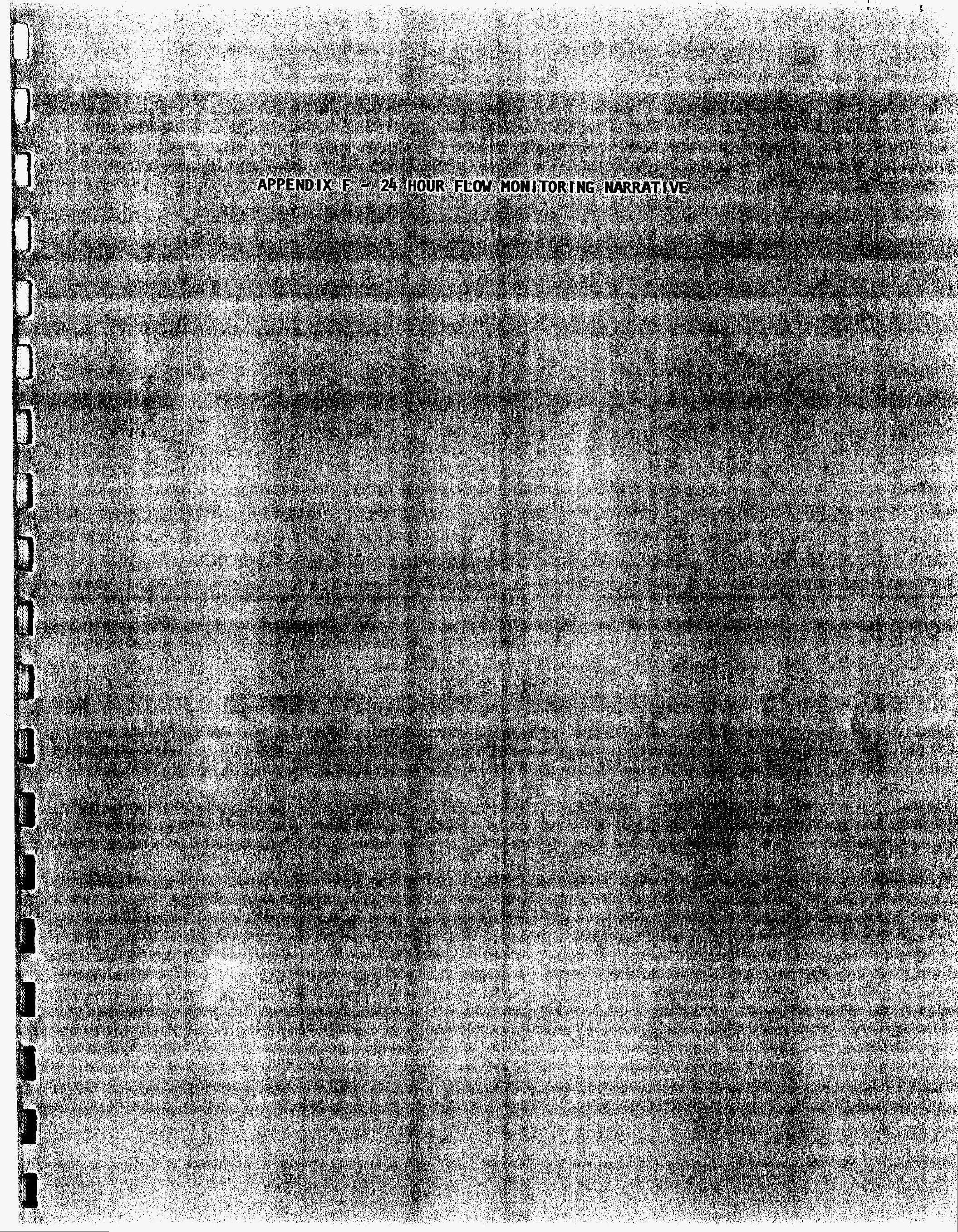




\section{LOCKWDOD GREENE ARCHITECTS - ENGINEERS 1330 WEST PEACHTREE STREET N.W. \\ ATLANTA, GEORGIA 30367-6801}

PROJECT DuPont Flow Study

\section{MEETING MINUTES}

MEETING NO.

PAGE

PROJECT NO.

83251.04

DATE

DATE

No.

Attendees

Copies

Attendees

No.

Copies

Hank Drannon, DuPont

Pete Smith, LGE

Bill Cook, LGE

Steve Morton, LGE

Gary Gelderman, LGE

Other distribution:

Attendees: Please inform the writer of required corrections or additions to these minutes.

\section{SUB JECT}

We met at the site on 10/4 at 1:20 p.m. to go over details of our flow measurement and sample taking, etc. Gary obtained temp. badge.

Hank gave us a beeper, 3 pairs of leggings and made arrangements for rubber gloves and boots to be picked up at Steed'd Pond on 10/5 a.m.

LGE dropped off equipment at each site (10/4) in preparation for start up on 10/5. On 10/5 a.m. LGE started calibration, etc. of each machine. At 2:00 p.m. all flow measuring and sample devices were ready. A problem at site 1 with the dam required some lowering of weir plate and shifting of sand bags. At 2:00 p.m. all 4 LGE people were involved in initializing of the devices. Morton and Cook left after the initial run and then returned to hotel. Smith and Gelderman took first 12 hour shift which began at 2:00 p.m. on 10/5. During the first shift the yardstick depth was adjusted at sites 2 and 4 . At 9:30 p.m. a slight shower occurred

\section{ACTION BY}

DUE DATE 
totaling only a trace of rain on the order of .01" to. l" $^{\prime \prime}$ of water. No evidence of runoff was apparent and therefore no effect on the streams or ground water is expected.

At $1: 30$ a.m. (10/6) Morton and Cook returned to take the second 12 hour shift. During this joint inspection, it became obvious that several malfunctions were occurring.

Site 1 - Pumped only air due to apparent loss of priming and no water had accumulated in the sample jar. Flow recorder pen was apparently stuck also.

Site 2 - Flow recorder gear was stuck and chart not moving. site 3 - Battery had run down.

Site 4 - No problems noted.

Site 5 - No water had accumulated in the sample jar ( as on site 1) and battery was showing signs of weakness.

At this point it was decided that we would probably have to amend the way composite sampling was done and rely on the depth readings for the flows.

Smith and Gelderman returned at 1:00 p.m. to assist in pickup and packing of samples and equipment. During pickup at Steed's Pond Smith walked downstream of the site and noted a previously unnoticed tributary from Steed's Pond. The flow was estimated to be $15 \mathrm{gpm}$ by a field estimate of a $5^{\prime \prime} \times 1 / 2^{\prime \prime}$ cross section at $2 \mathrm{ft} / \mathrm{sec}=.034 \mathrm{cfs}$ or $15 \mathrm{gpm}$. Since this flow is about $100^{\prime}$ downstream and represents other flow around or thru Steed's Pond it should be considered constant and added to the weir flows at site 4 .

DuPont had asked LGE to determine if any surface water existed in the creek bed located about $2000^{\prime}$ south of the treatment plant area and draining toward the east. At 3:30 p.m. (10/6) Smith and Gelderman traveled in the creek bed approximately $2000^{\prime}$ but found no surface water.

All 4 LGE people met at the hotel to obtain chilled gel packs and to go to UPS for packing and shipping. Shipping was complete at $6: 15 \mathrm{p} . \mathrm{m}$. 


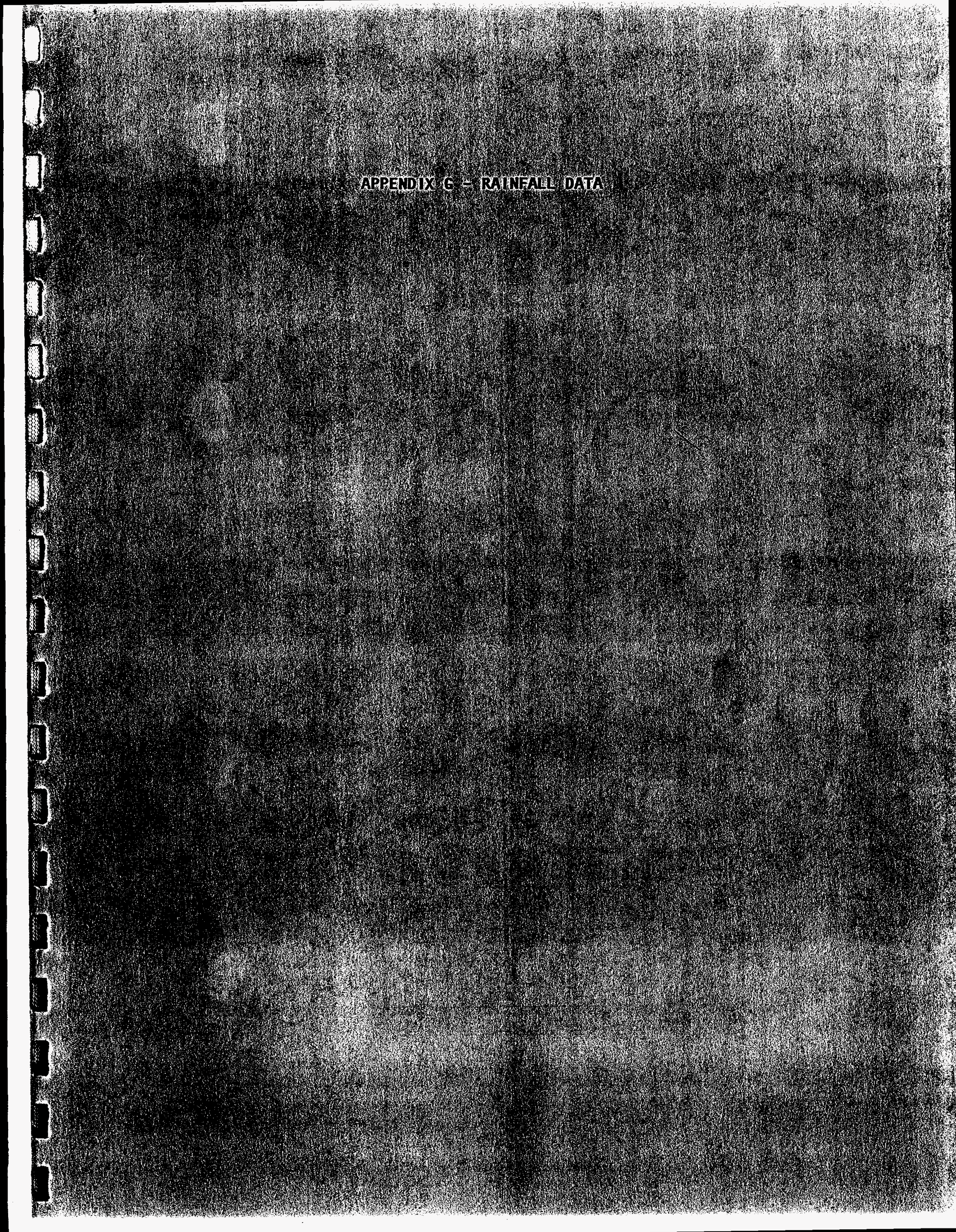


LOCKWOOD GAEENE

ARCHITECTS - ENGINEERS

ATLANTA

PR OJECT DUPONT STUDY

JOB NO. 83251.04

TEL. BILLING

TOPIC

Mr. Drannon connected me with Mark Kennedy of the Meterologic Group

in charge of maintaining rainfall data. The data showed the

following rainfall on the dates indicated:

13 Sept. 83 - 2.07" Rainfall

20 Sept. $83-0.88^{\prime \prime}$ Rainfall

21 Sept. 83 - 0.01 " Rainfall

5 oct. 83 - 0.02" Rainfall

This indicates a 14 day dry period between 20 sept. 83 and 5 oct. 83

if the .01" rainfall is ignored.
DATE $\quad 10-28-83$

TIME 3:15 pm

PAR TIES H. Drannon, M. Kennedy -Dupont P. Smith - IGE

SKETCH

$$
\text { P.J. Smith }
$$


UNITED STATES DEPAHTMENT OF INTERIOR - GEDLOGICAL SURVEY

\begin{tabular}{|c|c|c|c|c|c|c|}
\hline $\begin{array}{l}\text { STATION NUMBER } \\
\text { LATITUDE } 331712\end{array}$ & $\begin{array}{l}02197309 \\
\text { LUNGTTUDE. }\end{array}$ & $\begin{array}{l}\text { TIMS BRANCH A } \\
\text { OS14145 }\end{array}$ & $\begin{array}{l}\text { TOAD C (SRP) } \\
\text { DRAINAGE AREA }\end{array}$ & DATUM & STREAM & $\begin{array}{l}\text { SOIIRCE } \\
\text { STAENCY USGS } \\
\text { STATF. } 45 \text { COUNTY DO3 }\end{array}$ \\
\hline
\end{tabular}

DISCHARGE, IN CUBIC FFET PER SECONO. WATER YEAR OCTOBER 1973 TO SEPTEMBER 1974

\begin{tabular}{|c|c|c|c|c|c|c|c|c|c|c|c|c|}
\hline $\begin{array}{r}D A Y \\
1 \\
1 \\
2 \\
3 \\
4 \\
5\end{array}$ & OCT & NOV & DEC & JAN & FER & \begin{tabular}{c} 
MAR \\
$\vdots$ \\
\hdashline- \\
\hdashline$-\infty$ \\
\hdashline-
\end{tabular} & $\begin{array}{l}\text { APR } \\
5.9 \\
6.4 \\
6.2 \\
6.2 \\
17\end{array}$ & $\begin{array}{l}\text { MAY } \\
4.6 \\
4.6 \\
4.5 \\
4.8 \\
6.8\end{array}$ & $\begin{array}{l}\text { JUN } \\
4.3 \\
4.6 \\
4.5 \\
4.3 \\
4.3\end{array}$ & $\begin{array}{r}\text { JUL } \\
\begin{array}{r}3.5 \\
3.5\end{array} \\
4.3 \\
8.9 \\
8.5\end{array}$ & $\begin{array}{l}\text { AUG } \\
3.8 \\
4.5 \\
4.7 \\
5.1 \\
8.1\end{array}$ & $\begin{array}{l}\text { SEP } \\
4.9 \\
5.5 \\
6.3 \\
5.0 \\
5.0\end{array}$ \\
\hline $\begin{array}{r}6 \\
7 \\
8 \\
9 \\
10\end{array}$ & & & & & & 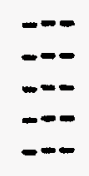 & $\begin{array}{l}8.5 \\
6.6 \\
9.0 \\
9.7 \\
7.0\end{array}$ & $\begin{array}{l}5.5 \\
5.1 \\
4.8 \\
4.7 \\
4.6\end{array}$ & $\begin{array}{l}4.5 \\
5.3 \\
9.8 \\
5.1 \\
4.5\end{array}$ & $\begin{array}{l}6.2 \\
7.7 \\
5.0 \\
4.4 \\
4.3\end{array}$ & $\begin{array}{l}13 \\
7.8 \\
7.0 \\
5.8 \\
5.5\end{array}$ & $\begin{array}{l}15 \\
13 \\
7.0 \\
5.5 \\
5.0\end{array}$ \\
\hline $\begin{array}{l}11 \\
12 \\
13 \\
14 \\
15\end{array}$ & & & & & & $=-$ & $\begin{array}{l}6.4 \\
6.1 \\
6.0 \\
6.2 \\
6.7\end{array}$ & $\begin{array}{l}4.5 \\
6.3 \\
5.5 \\
5.0 \\
4.7\end{array}$ & $\begin{array}{l}4.7 \\
4.3 \\
4.1 \\
4.1 \\
5.1\end{array}$ & $\begin{array}{l}4.1 \\
4.0 \\
3.9 \\
3.7 \\
3.6\end{array}$ & $\begin{array}{l}9.7 \\
6.2 \\
6.9 \\
7.8 \\
5.9\end{array}$ & $\begin{array}{l}4.8 \\
4.5 \\
4.4 \\
4.7 \\
4.7\end{array}$ \\
\hline $\begin{array}{l}16 \\
17 \\
18 \\
19 \\
20\end{array}$ & & & & & & $\begin{array}{l}--- \\
--- \\
--\infty .8 \\
7.8\end{array}$ & $\begin{array}{l}5.9 \\
5.6 \\
5.5 \\
5.5 \\
5.3\end{array}$ & $\begin{array}{l}4.8 \\
4.7 \\
4.4 \\
6.8 \\
11\end{array}$ & $\begin{array}{l}4.6 \\
4.3 \\
4.1 \\
4.0 \\
4.1\end{array}$ & $\begin{array}{l}3.6 \\
6.2 \\
5.2 \\
4.0 \\
3.9\end{array}$ & $\begin{array}{l}5.1 \\
4.7 \\
4.5 \\
4.2 \\
4.2\end{array}$ & $\begin{array}{l}4.3 \\
5.1 \\
6.4 \\
5.1 \\
4.6\end{array}$ \\
\hline $\begin{array}{l}21 \\
22 \\
23 \\
24 \\
25\end{array}$ & & & & & & $\begin{array}{l}12 \\
8.2 \\
6.7 \\
6.4 \\
9.0\end{array}$ & $\begin{array}{l}5.1 \\
5.1 \\
5.6 \\
5.2 \\
5.0\end{array}$ & $\begin{array}{l}6.5 \\
5.7 \\
5.7 \\
5.3 \\
4.8\end{array}$ & $\begin{array}{l}4.1 \\
3.9 \\
3.9 \\
3.9 \\
3.8\end{array}$ & $\begin{array}{l}4.0 \\
3.9 \\
3.8 \\
4.7 \\
4.6\end{array}$ & $\begin{array}{l}4.5 \\
4.3 \\
4.2 \\
4.2 \\
4.2\end{array}$ & $\begin{array}{l}4.4 \\
4.7 \\
4.9 \\
4.2 \\
4.2\end{array}$ \\
\hline $\begin{array}{l}26 \\
27 \\
28 \\
29 \\
30 \\
31\end{array}$ & & & & & & $\begin{array}{l}7.7 \\
6.8 \\
6.6 \\
8.5 \\
7.2 \\
6.1\end{array}$ & $\begin{array}{l}4.9 \\
4.8 \\
4.7 \\
4.7 \\
4.6 \\
-.-\end{array}$ & $\begin{array}{l}5.1 \\
7.0 \\
5.2 \\
4.9 \\
4.7 \\
4.4\end{array}$ & $\begin{array}{l}3.9 \\
4.0 \\
3.9 \\
3.8 \\
3.6 \\
0.0\end{array}$ & $\begin{array}{l}4.3 \\
4.2 \\
5.8 \\
4.3 \\
3.9 \\
3.8\end{array}$ & $\begin{array}{l}4.2 \\
4.1 \\
4.5 \\
7.5 \\
6.1 \\
5.0\end{array}$ & $\begin{array}{l}4.3 \\
4.4 \\
4.4 \\
4.2 \\
3.9 \\
-.0\end{array}$ \\
\hline $\begin{array}{l}\text { TOTAL } \\
\text { MFAN } \\
\text { MAX } \\
\text { MIN }\end{array}$ & & & & & & 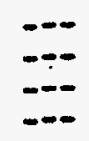 & $\begin{array}{r}191.4 \\
6.38 \\
17 \\
4.6\end{array}$ & $\begin{array}{r}167.0 \\
5.39 \\
11 \\
4.4\end{array}$ & $\begin{array}{r}133.4 \\
4.45 \\
9.8 \\
3.6\end{array}$ & $\begin{array}{r}145.8 \\
4.70 \\
8.9 \\
3.5\end{array}$ & $\begin{array}{r}177.3 \\
5.72 \\
13 \\
3.8\end{array}$ & $\begin{array}{r}164.4 \\
5.48 \\
15 \\
3.9\end{array}$ \\
\hline
\end{tabular}


UNITED STATES DEPARTMENT OF INTERIOR - GEOLOGICAL SURVEY

PROCESS DATE IS 10-03-83

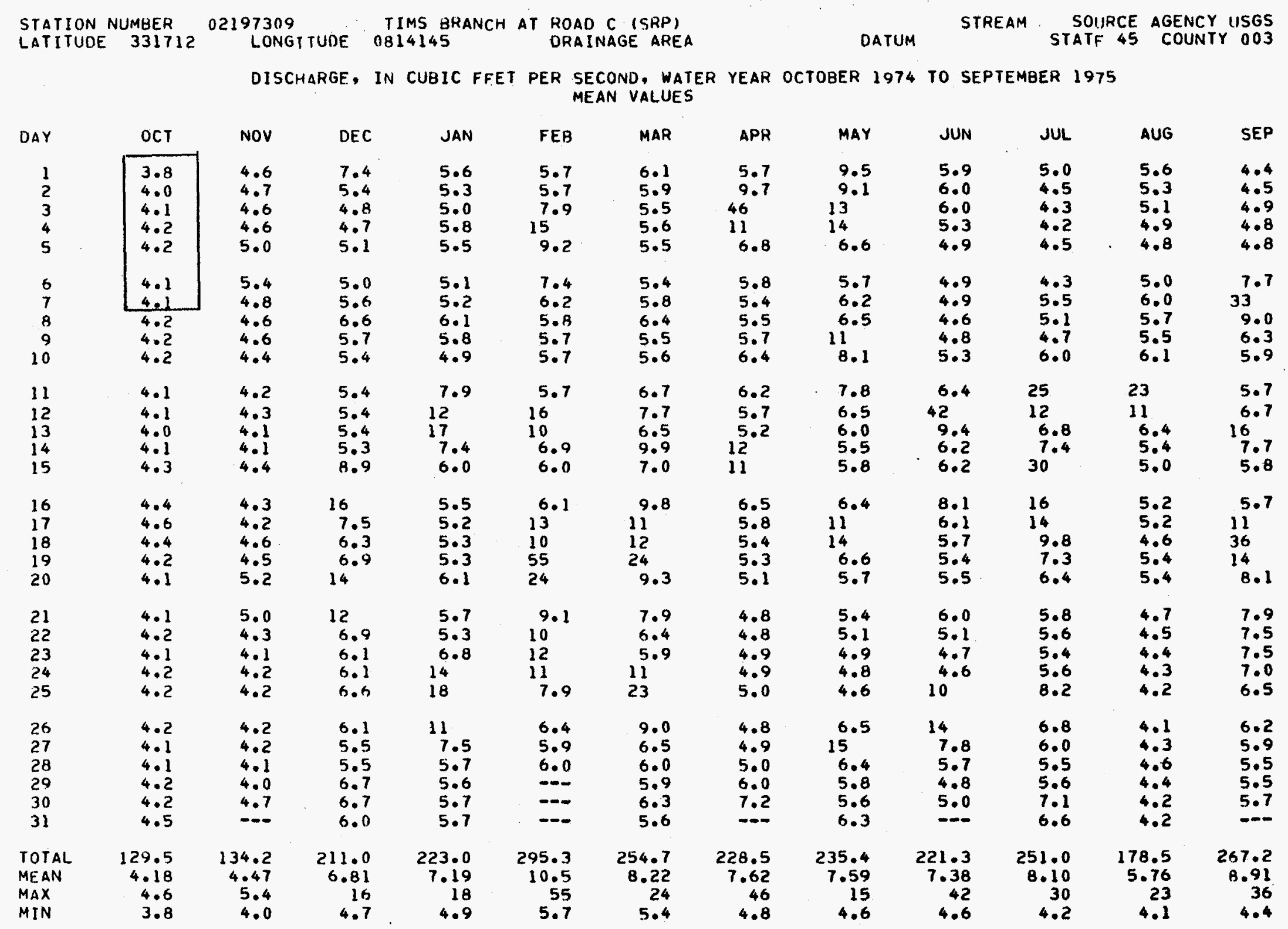

WTR YR 1975 TOTAL 2629.6 MEAN 7.20 MAX 55 MIN 3.8 
UNITED : STATES DEPAHTMENT OF INTERIOR - GEOLUGICAL SURVEY

PROCESS DATE IS 10-03-83

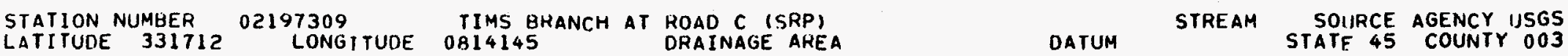
DISCHARGE. IN CUBIC FFET PER SECOND, WATER YEAR OCTOBER 1975 TO SEPTEMBER 1976 MEAN VALUES

\begin{tabular}{|c|c|c|c|c|c|c|c|c|c|c|c|c|c|c|}
\hline DAY & OCT & Nov & DEC & JAN & & FEB & & MAR & APR & MAY & JUN & JUL & AUG & SEP \\
\hline $\begin{array}{l}1 \\
2 \\
3 \\
4 \\
5\end{array}$ & $\begin{array}{l}6.3 \\
6.1 \\
5.1 \\
5.0 \\
4.9\end{array}$ & $\begin{array}{l}4.6 \\
4.6 \\
4.6 \\
4.8 \\
5.0\end{array}$ & $\begin{array}{l}7.1 \\
6.9 \\
6.4 \\
6.3 \\
6.2\end{array}$ & $\begin{array}{c}12 \\
7.8 \\
7.1 \\
6.8 \\
6.1\end{array}$ & & $\begin{array}{l}8.6 \\
9.2 \\
8.8 \\
7.5 \\
7.3\end{array}$ & & $\begin{array}{l}5.2 \\
5.5 \\
5.3 \\
5.2 \\
5.3\end{array}$ & $\begin{array}{l}5.5 \\
5.1 \\
4.9 \\
4.7 \\
4.7\end{array}$ & $\begin{array}{l}13 \\
6.3 \\
4.8 \\
4.4 \\
4.3\end{array}$ & $\begin{array}{c}9.1 \\
7.2 \\
9.3 \\
12 \\
8.1\end{array}$ & $\begin{array}{l}5.1 \\
4.6 \\
4.4 \\
5.5 \\
7.5\end{array}$ & \begin{tabular}{|l|}
3.8 \\
3.7 \\
3.6 \\
3.7 \\
3.7
\end{tabular} & $\begin{array}{l}3.7 \\
3.9 \\
4.0 \\
4.1 \\
4.1\end{array}$ \\
\hline $\begin{array}{r}6 \\
7 \\
8 \\
9 \\
10\end{array}$ & $\begin{array}{l}4.9 \\
4.9 \\
5.5 \\
9.2 \\
6.5\end{array}$ & $\begin{array}{l}5.0 \\
6.3 \\
6.6 \\
5.5 \\
5.7\end{array}$ & $\begin{array}{c}6.0 \\
5.8 \\
6.1 \\
11 \\
9.4\end{array}$ & $\begin{array}{l}6.3 \\
12 \\
28 \\
11 \\
8.6\end{array}$ & & $\begin{array}{l}7.0 \\
6.6 \\
6.1 \\
5.9 \\
6.3\end{array}$ & & $\begin{array}{l}5.3 \\
5.1 \\
5.4 \\
8.3 \\
6.2\end{array}$ & $\begin{array}{l}4.9 \\
4.9 \\
4.8 \\
4.7 \\
4.5\end{array}$ & $\begin{array}{l}4.2 \\
4.2 \\
4.8 \\
4.9 \\
4.2\end{array}$ & $\begin{array}{l}6.1 \\
5.4 \\
5.3 \\
7.6 \\
7.8\end{array}$ & $\begin{array}{l}7.4 \\
6.3 \\
5.2 \\
4.7 \\
4.5\end{array}$ & $\begin{array}{l}3.7 \\
3.7 \\
3.6 \\
3.8 \\
4.2\end{array}$ & $\begin{array}{l}4.2 \\
4.1 \\
4.0 \\
4.0 \\
4.0\end{array}$ \\
\hline $\begin{array}{l}11 \\
12 \\
13 \\
14 \\
15\end{array}$ & $\begin{array}{l}5.6 \\
5.1 \\
5.0 \\
5.0 \\
5.0\end{array}$ & $\begin{array}{l}13 \\
24 \\
32 \\
10 \\
7.1\end{array}$ & $\begin{array}{l}7.3 \\
6.5 \\
6.3 \\
6.0 \\
5.8\end{array}$ & $\begin{array}{l}7.5 \\
7.1 \\
7.0 \\
6.9 \\
6.5\end{array}$ & & $\begin{array}{l}6.6 \\
6.3 \\
6.1 \\
6.2 \\
5.8\end{array}$ & & $\begin{array}{l}5.5 \\
5.5 \\
9.4 \\
6.2 \\
10\end{array}$ & $\begin{array}{l}4.5 \\
4.4 \\
4.5 \\
4.7 \\
4.8\end{array}$ & $\begin{array}{l}4.3 \\
4.4 \\
4.1 \\
4.1 \\
7.9\end{array}$ & $\begin{array}{l}5.9 \\
6.9 \\
5.6 \\
5.2 \\
5.2\end{array}$ & $\begin{array}{l}4.3 \\
4.1 \\
4.0 \\
3.9 \\
3.9\end{array}$ & $\begin{array}{l}5.0 \\
4.4 \\
4.1 \\
4.0 \\
4.0\end{array}$ & $\begin{array}{r}3.8 \\
3.8 \\
3.9 \\
23 \\
30\end{array}$ \\
\hline $\begin{array}{l}16 \\
17 \\
18 \\
19 \\
20\end{array}$ & $\begin{array}{l}4.9 \\
6.0 \\
6.6 \\
5.2 \\
5.1\end{array}$ & $\begin{array}{l}6.1 \\
5.8 \\
6.0 \\
6.2 \\
6.2\end{array}$ & $\begin{array}{l}7.2 \\
12 \\
12 \\
7.9 \\
6.9\end{array}$ & $\begin{array}{l}6.4 \\
7.3 \\
6.7 \\
6.1 \\
6.3\end{array}$ & & $\begin{array}{l}5.7 \\
5.9 \\
6.7 \\
8.6 \\
6.8\end{array}$ & & $\begin{array}{l}39 \\
17 \\
13 \\
8.3 \\
6.8\end{array}$ & $\begin{array}{l}4.6 \\
4.3 \\
4.1 \\
4.0 \\
4.2\end{array}$ & $\begin{array}{l}6.5 \\
6.3 \\
5.0 \\
4.4 \\
4.2\end{array}$ & $\begin{array}{l}6.4 \\
7.2 \\
6.7 \\
5.9 \\
6.4\end{array}$ & $\begin{array}{l}3.8 \\
3.8 \\
4.1 \\
4.0 \\
3.9\end{array}$ & $\begin{array}{l}3.9 \\
4.0 \\
3.9 \\
3.7 \\
4.1\end{array}$ & $\begin{array}{r}11 \\
5.9 \\
4.9 \\
4.8 \\
4.7\end{array}$ \\
\hline $\begin{array}{l}21 \\
22 \\
23 \\
24 \\
25\end{array}$ & $\begin{array}{l}4.9 \\
4.9 \\
4.9 \\
4.9 \\
4.9\end{array}$ & $\begin{array}{l}6.1 \\
5.9 \\
5.6 \\
5.7 \\
6.1\end{array}$ & $\begin{array}{l}6.3 \\
6.2 \\
6.1 \\
5.9 \\
6.0\end{array}$ & $\begin{array}{l}6.5 \\
6.3 \\
6.2 \\
6.2 \\
5.9\end{array}$ & & $\begin{array}{l}6.3 \\
6.5 \\
5.9 \\
5.6 \\
5.6\end{array}$ & & $\begin{array}{l}6.1 \\
5.5 \\
5.4 \\
5.3 \\
5.3\end{array}$ & $\begin{array}{l}4.3 \\
5.5 \\
4.6 \\
4.3 \\
4.2\end{array}$ & $\begin{array}{l}4.0 \\
3.9 \\
5.9 \\
12 \\
11\end{array}$ & $\begin{array}{l}6.5 \\
5.6 \\
5.4 \\
5.6 \\
5.4\end{array}$ & $\begin{array}{l}3.8 \\
3.8 \\
4.0 \\
3.9 \\
3.8\end{array}$ & $\begin{array}{l}4.8 \\
4.5 \\
4.3 \\
4.1 \\
4.2\end{array}$ & $\begin{array}{l}4.8 \\
4.5 \\
4.3 \\
4.3 \\
4.2\end{array}$ \\
\hline $\begin{array}{l}26 \\
27 \\
28 \\
29 \\
30 \\
31\end{array}$ & $\begin{array}{l}4.9 \\
4.8 \\
4.8 \\
5.0 \\
4.9 \\
4.6\end{array}$ & $\begin{array}{l}6.1 \\
6.2 \\
5.9 \\
5.8 \\
5.8 \\
-\ldots\end{array}$ & $\begin{array}{c}13 \\
8.1 \\
6.9 \\
6.4 \\
8.4 \\
15\end{array}$ & $\begin{array}{l}7.5 \\
39 \\
17 \\
11 \\
8.1 \\
7.2\end{array}$ & & $\begin{array}{l}5.6 \\
5.5 \\
5.4 \\
5.3 \\
-.-\end{array}$ & & $\begin{array}{l}5.5 \\
5.7 \\
5.3 \\
5.2 \\
5.4 \\
5.5\end{array}$ & $\begin{array}{r}4.1 \\
4.1 \\
4.0 \\
4.0 \\
17 \\
\end{array}$ & $\begin{array}{l}6.3 \\
8.7 \\
36 \\
86 \\
26 \\
16\end{array}$ & $\begin{array}{l}4.9 \\
4.6 \\
5.1 \\
5.0 \\
5.8 \\
--.\end{array}$ & $\begin{array}{l}3.9 \\
3.8 \\
4.1 \\
4.2 \\
3.9 \\
3.9\end{array}$ & $\begin{array}{l}4.3 \\
4.2 \\
4.1 \\
4.0 \\
3.9 \\
3.7\end{array}$ & $\begin{array}{l}4.6 \\
4.8 \\
4.8 \\
5.0 \\
5.4 \\
-\end{array}$ \\
\hline $\begin{array}{l}\text { TOTAL } \\
\text { ME.AN } \\
\text { MAX } \\
\text { MIN }\end{array}$ & $\begin{array}{r}165.4 \\
5.34 \\
9.2 \\
4.6\end{array}$ & $\begin{array}{r}228.3 \\
7.61 \\
32 \\
4.6\end{array}$ & $\begin{array}{r}237.4 \\
7.66 \\
15 \\
5.8\end{array}$ & $\begin{array}{r}294.4 \\
9.50 \\
39 \\
5.9\end{array}$ & & $\begin{array}{r}189.7 \\
6.54 \\
9.2 \\
5.3\end{array}$ & & $\begin{array}{r}237.7 \\
7.67 \\
39 \\
5.1\end{array}$ & $\begin{array}{r}148.9 \\
4.96 \\
17 \\
4.0\end{array}$ & $\begin{array}{r}322.1 \\
10.4 \\
86 \\
3.9\end{array}$ & $\begin{array}{r}193.2 \\
6.44 \\
12 \\
4.6\end{array}$ & $\begin{array}{r}138.1 \\
4.45 \\
7.5 \\
3.8\end{array}$ & $\begin{array}{r}124.7 \\
4.02 \\
5.0 \\
3.6\end{array}$ & $\begin{array}{r}182.6 \\
6.09 \\
30 \\
3.7\end{array}$ \\
\hline
\end{tabular}


UNITEO STATES DEPARTMENT OF INTERIOR - GEOLOGICAL SURVEY

PROCESS DATE IS $10-03-83$

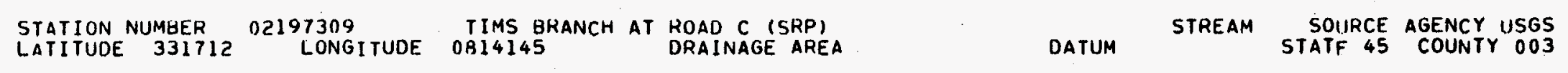
DISCHARGE, IN CUBIC FFET PER SECOND, WATER YEAR OCTOBER 1976 TO SEPTEMBER 1977
MEAN VALUES

\begin{tabular}{|c|c|c|c|c|c|c|c|c|c|c|c|c|}
\hline DAY & OCT & NOV & DEC & JAN & FEB & MAR & APR & MAY & JUN & JUL & AUG & SEP \\
\hline $\begin{array}{l}1 \\
2 \\
3 \\
4 \\
5\end{array}$ & $\begin{array}{l}4.9 \\
4.5 \\
4.3 \\
4.2 \\
4.1\end{array}$ & $\begin{array}{l}7.2 \\
6.9 \\
5.9 \\
5.8 \\
5.4\end{array}$ & $\begin{array}{l}7.4 \\
6.9 \\
6.2 \\
4.7 \\
4.6\end{array}$ & $\begin{array}{l}3.7 \\
3.4 \\
4.4 \\
5.2 \\
4.4\end{array}$ & $\begin{array}{l}3.8 \\
3.5 \\
3.6 \\
3.9 \\
3.8\end{array}$ & $\begin{array}{l}6.2 \\
5.5 \\
5.4 \\
15 \\
11\end{array}$ & $\begin{array}{l}6.6 \\
6.3 \\
.6 .1 \\
5.6 \\
7.2\end{array}$ & $\begin{array}{l}4.9 \\
4.9 \\
4.8 \\
4.8 \\
4.8\end{array}$ & $\begin{array}{l}4.3 \\
4.1 \\
4.1 \\
4.1 \\
4.1\end{array}$ & $\begin{array}{l}3.5 \\
3.5 \\
3.5 \\
3.5 \\
3.3\end{array}$ & $\begin{array}{c}5.3 \\
4.8 \\
16 \\
13 \\
6.6\end{array}$ & $\begin{array}{l}3.9 \\
3.7 \\
3.8 \\
4.9 \\
4.9\end{array}$ \\
\hline $\begin{array}{r}6 \\
7 \\
8 \\
9 \\
10\end{array}$ & $\begin{array}{c}4.1 \\
4.3 \\
6.5 \\
13 \\
5.8\end{array}$ & $\begin{array}{l}5.0 \\
4.9 \\
4.8 \\
5.1 \\
5.2\end{array}$ & $\begin{array}{l}4.9 \\
9.9 \\
6.0 \\
6.2 \\
5.9\end{array}$ & $\begin{array}{l}4.1 \\
4.6 \\
3.5 \\
3.2 \\
5.8\end{array}$ & $\begin{array}{l}3.7 \\
4.0 \\
4.2 \\
4.4 \\
4.4\end{array}$ & $\begin{array}{l}17 \\
22 \\
11 \\
9.3 \\
8.7\end{array}$ & $\begin{array}{l}5.8 \\
5.3 \\
5.4 \\
5.4 \\
5.4\end{array}$ & $\begin{array}{l}4.6 \\
4.8 \\
4.9 \\
4.7 \\
4.5\end{array}$ & $\begin{array}{l}4.0 \\
3.9 \\
3.8 \\
3.7 \\
3.6\end{array}$ & $\begin{array}{l}3.4 \\
3.1 \\
3.0 \\
2.9 \\
2.8\end{array}$ & $\begin{array}{l}5.9 \\
5.1 \\
4.4 \\
4.0 \\
3.8\end{array}$ & $\begin{array}{c}4.2 \\
7.4 \\
12 \\
6.4 \\
4.9\end{array}$ \\
\hline $\begin{array}{l}11 \\
12 \\
13 \\
14 \\
15\end{array}$ & $\begin{array}{l}4.8 \\
4.5 \\
4.4 \\
4.3 \\
4.2\end{array}$ & $\begin{array}{l}5.2 \\
5.0 \\
4.8 \\
5.1 \\
10\end{array}$ & $\begin{array}{l}7.9 \\
24 \\
16 \\
9.7 \\
8.9\end{array}$ & $\begin{array}{l}3.9 \\
3.7 \\
3.9 \\
5.9 \\
4.8\end{array}$ & $\begin{array}{l}6.6 \\
5.4 \\
5.3 \\
5.1 \\
5.1\end{array}$ & $\begin{array}{r}8.3 \\
7.6 \\
15 \\
7.2 \\
6.5\end{array}$ & $\begin{array}{r}5.4 \\
5.3 \\
5.1 \\
11.2 \\
7.2\end{array}$ & $\begin{array}{l}4.6 \\
4.5 \\
4.5 \\
4.4 \\
4.4\end{array}$ & $\begin{array}{l}3.6 \\
4.4 \\
3.8 \\
4.0 \\
3.1\end{array}$ & $\begin{array}{l}2.8 \\
2.8 \\
3.1 \\
3.2 \\
3.0\end{array}$ & $\begin{array}{l}3.6 \\
3.4 \\
3.4 \\
3.7 \\
4.0\end{array}$ & $\begin{array}{l}4.4 \\
4.1 \\
4.0 \\
4.1 \\
4.1\end{array}$ \\
\hline $\begin{array}{l}16 \\
17 \\
18 \\
19 \\
20\end{array}$ & $\begin{array}{l}4.3 \\
8.1 \\
6.6 \\
5.0 \\
24\end{array}$ & $\begin{array}{l}7.6 \\
7.6 \\
7.3 \\
6.8 \\
6.3\end{array}$ & $\begin{array}{l}7.9 \\
6.9 \\
6.5 \\
5.8 \\
6.0\end{array}$ & $\begin{array}{l}4.2 \\
4.5 \\
4.4 \\
4.3 \\
4.4\end{array}$ & $\begin{array}{l}5.0 \\
5.9 \\
6.4 \\
5.8 \\
5.2\end{array}$ & $\begin{array}{l}6.3 \\
11 \\
10 \\
6.5 \\
7.3\end{array}$ & $\begin{array}{l}5.3 \\
5.1 \\
5.1 \\
5.1 \\
5.1\end{array}$ & $\begin{array}{l}4.3 \\
4.9 \\
4.2 \\
3.8 \\
4.2\end{array}$ & $\begin{array}{l}3.4 \\
3.5 \\
3.5 \\
3.3 \\
3.4\end{array}$ & $\begin{array}{l}2.8 \\
2.8 \\
3.3 \\
3.1 \\
3.0\end{array}$ & $\begin{array}{l}8.7 \\
5.4 \\
5.1 \\
5.7 \\
5.4\end{array}$ & $\begin{array}{l}4.1 \\
4.7 \\
4.2 \\
4.1 \\
4.1\end{array}$ \\
\hline $\begin{array}{l}21 \\
22 \\
23 \\
24 \\
25\end{array}$ & $\begin{array}{l}11 \\
9.0 \\
7.6 \\
7.5 \\
8.3\end{array}$ & $\begin{array}{l}5.9 \\
5.2 \\
4.9 \\
4.9 \\
5.0\end{array}$ & $\begin{array}{l}5.8 \\
5.7 \\
5.8 \\
5.0 \\
5.1\end{array}$ & $\begin{array}{l}4.4 \\
4.8 \\
3.9 \\
4.5 \\
4.7\end{array}$ & $\begin{array}{r}5.0 \\
5.0 \\
5.1 \\
12 \\
7.0\end{array}$ & $\begin{array}{l}9.3 \\
36 \\
11 \\
10 \\
9.2\end{array}$ & $\begin{array}{l}5.0 \\
5.4 \\
7.1 \\
6.7 \\
5.6\end{array}$ & $\begin{array}{l}4.1 \\
4.3 \\
4.3 \\
5.3 \\
5.1\end{array}$ & $\begin{array}{l}3.3 \\
3.1 \\
3.1 \\
3.1 \\
3.0\end{array}$ & $\begin{array}{l}3.0 \\
3.4 \\
3.7 \\
3.3 \\
3.2\end{array}$ & $\begin{array}{r}5.2 \\
4.8 \\
4.7 \\
7.1 \\
21\end{array}$ & $\begin{array}{l}4.0 \\
3.9 \\
3.8 \\
3.9 \\
3.9\end{array}$ \\
\hline $\begin{array}{l}26 \\
27 \\
29 \\
29 \\
30 \\
31\end{array}$ & $\begin{array}{l}9.7 \\
7.9 \\
7.5 \\
7.4 \\
7.5 \\
7.4\end{array}$ & $\begin{array}{r}5.8 \\
7.2 \\
12 \\
9.2 \\
7.2 \\
---\end{array}$ & $\begin{array}{l}7.8 \\
5.9 \\
4.8 \\
4.5 \\
4.2 \\
4.0\end{array}$ & $\begin{array}{l}4.0 \\
3.7 \\
3.8 \\
4.3 \\
4.9 \\
3.7\end{array}$ & $\begin{array}{l}5.3 \\
9.5 \\
8.8 \\
--- \\
---\end{array}$ & $\begin{array}{l}\text { A.1 } \\
7.5 \\
6.9 \\
6.2 \\
7.7 \\
8.8\end{array}$ & $\begin{array}{l}5.2 \\
5.2 \\
5.1 \\
4.9 \\
5.0 \\
-.-\end{array}$ & $\begin{array}{l}4.9 \\
4.8 \\
4.6 \\
4.5 \\
4.4 \\
4.4\end{array}$ & $\begin{array}{l}3.7 \\
4.1 \\
3.3 \\
3.6 \\
3.7 \\
-\end{array}$ & $\begin{array}{l}3.5 \\
3.5 \\
3.4 \\
4.0 \\
4.5 \\
4.5\end{array}$ & $\begin{array}{l}39 \\
9.9 \\
6.0 \\
5.3 \\
4.9 \\
4.2\end{array}$ & $\begin{array}{r}8.1 \\
7.4 \\
11 \\
6.2 \\
5.6 \\
\end{array}$ \\
\hline $\begin{array}{l}\text { TOTAL } \\
\text { MEAN } \\
\text { MAX } \\
\text { MIN }\end{array}$ & $\begin{array}{r}216.7 \\
6.99 \\
24 \\
4.1\end{array}$ & $\begin{array}{r}189.2 \\
6.31 \\
12 \\
4.8\end{array}$ & $\begin{array}{r}220.9 \\
7.13 \\
24 \\
4.0\end{array}$ & $\begin{array}{r}133.0 \\
4.29 \\
5.9 \\
3.2\end{array}$ & $\begin{array}{r}152.8 \\
5.46 \\
12 \\
3.5\end{array}$ & $\begin{array}{r}317.5 \\
10.2 \\
36 \\
5.4\end{array}$ & $\begin{array}{r}174.0 \\
5.80 \\
11 \\
4.9\end{array}$ & $\begin{array}{r}142.2 \\
4.59 \\
5.3 \\
3.8\end{array}$ & $\begin{array}{r}109.7 \\
3.66 \\
4.4 \\
3.0\end{array}$ & $\begin{array}{r}102.4 \\
3.30 \\
4.5 \\
2.8\end{array}$ & $\begin{array}{r}229.4 \\
7.40 \\
39 \\
3.4\end{array}$ & $\begin{array}{r}155.8 \\
5.19 \\
12 \\
3.7\end{array}$ \\
\hline
\end{tabular}


INITED STATES DEPARTMENT OF INTERIOR - GEOLOGICAL SURVEY

PROCESS DATE IS 10-03-83

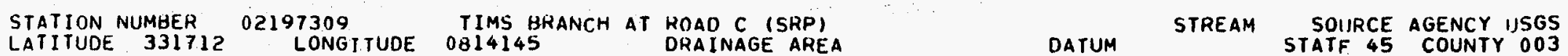
DISCHARGE, IN CUBIC FEET PER SECOND. WATER YEAR OCTOBER 1977 TO SEPTEMBER 1978 MEAN VALUES

\begin{tabular}{|c|c|c|c|c|c|c|c|c|c|c|c|c|}
\hline DAY & OCT & NOV & DEC & JAN & FEB & MAR & APR & MAY & JUN & JUL & AUG & SEP \\
\hline $\begin{array}{l}1 \\
2 \\
3 \\
4 \\
5\end{array}$ & $\begin{array}{l}5.0 \\
10 \\
11 \\
6.2 \\
6.2\end{array}$ & $\begin{array}{l}4.8 \\
5.0 \\
5.2 \\
5.6 \\
5.8\end{array}$ & $\begin{array}{l}25 \\
12 \\
8.0 \\
6.5 \\
22\end{array}$ & $\begin{array}{l}6.2 \\
5.1 \\
4.5 \\
5.1 \\
6.4\end{array}$ & $\begin{array}{l}8.4 \\
20 \\
21 \\
13 \\
8 . ?\end{array}$ & $\begin{array}{l}6.7 \\
6.2 \\
8.3 \\
7.2 \\
6.3\end{array}$ & $\begin{array}{l}5.3 \\
5.2 \\
5.1 \\
5.1 \\
5.1\end{array}$ & $\begin{array}{c}10 \\
8.6 \\
9.7 \\
12 \\
8.1\end{array}$ & $\begin{array}{c}4.1 \\
4.1 \\
4.5 \\
16 \\
9.0\end{array}$ & $\begin{array}{l}3.5 \\
3.4 \\
3.5 \\
3.3 \\
3.4\end{array}$ & $\left|\begin{array}{l}2.4 \\
2.4 \\
2.6 \\
2.6 \\
2.5\end{array}\right|$ & $\begin{array}{l}4.5 \\
4.7 \\
8.5 \\
5.5 \\
4.4\end{array}$ \\
\hline $\begin{array}{r}6 \\
7 \\
8 \\
9 \\
10\end{array}$ & $\begin{array}{l}7.1 \\
6.7 \\
12 \\
13 \\
7.5\end{array}$ & $\begin{array}{l}0.5 \\
5.7 \\
5.1 \\
5.1 \\
5.1\end{array}$ & $\begin{array}{l}12 \\
6.5 \\
5.7 \\
5.4 \\
5.0\end{array}$ & $\begin{array}{c}6.2 \\
5.3 \\
13 \\
28 \\
7.2\end{array}$ & $\begin{array}{l}7.5 \\
7.5 \\
7.4 \\
8.0 \\
7.8\end{array}$ & $\begin{array}{l}6.0 \\
5.9 \\
6.5 \\
12 \\
18\end{array}$ & $\begin{array}{l}5.0 \\
5.0 \\
4.8 \\
4.8 \\
4.8\end{array}$ & $\begin{array}{l}6.4 \\
5.5 \\
5.6 \\
25 \\
11\end{array}$ & $\begin{array}{l}8.2 \\
6.2 \\
6.9 \\
8.8 \\
6.0\end{array}$ & $\begin{array}{l}3.4 \\
3.3 \\
3.2 \\
3.0 \\
3.0\end{array}$ & $\begin{array}{l}2.6 \\
2.6 \\
2.4 \\
2.4 \\
3.4\end{array}$ & $\begin{array}{l}4.2 \\
4.3 \\
4.4 \\
9.5 \\
7.9\end{array}$ \\
\hline $\begin{array}{l}11 \\
12 \\
13 \\
14 \\
15\end{array}$ & $\begin{array}{l}5.1 \\
6.6 \\
6.9 \\
6.1 \\
5.5\end{array}$ & $\begin{array}{l}4.9 \\
4.6 \\
4.5 \\
4.5 \\
4.6\end{array}$ & $\begin{array}{l}4.4 \\
4.3 \\
4.3 \\
7.8 \\
7.4\end{array}$ & $\begin{array}{l}6.3 \\
6.7 \\
34 \\
17 \\
11\end{array}$ & $\begin{array}{l}7.2 \\
6.4 \\
7.3 \\
7.4 \\
6.2\end{array}$ & $\begin{array}{l}9.4 \\
7.1 \\
5.8 \\
5.7 \\
5.6\end{array}$ & $\begin{array}{c}4.9 \\
5.1 \\
16 \\
8.6 \\
6.4\end{array}$ & $\begin{array}{l}6.5 \\
5.9 \\
5.7 \\
5.1 \\
4.8\end{array}$ & $\begin{array}{l}5.0 \\
4.6 \\
4.4 \\
4.2 \\
4.0\end{array}$ & $\begin{array}{l}2.9 \\
2.9 \\
2.9 \\
2.8 \\
4.2\end{array}$ & $\begin{array}{l}5.0 \\
5.2 \\
4.7 \\
4.9 \\
3.6\end{array}$ & $\begin{array}{l}4.7 \\
3.9 \\
3.4 \\
3.2 \\
3.2\end{array}$ \\
\hline $\begin{array}{l}16 \\
17 \\
18 \\
19 \\
20\end{array}$ & $\begin{array}{l}4.8 \\
4.6 \\
4.4 \\
4.6 \\
4.6\end{array}$ & $\begin{array}{l}4.7 \\
4.8 \\
5.3 \\
5.3 \\
4.7\end{array}$ & $\begin{array}{l}5.1 \\
4.8 \\
5.6 \\
4.9 \\
5.6\end{array}$ & $\begin{array}{l}9.3 \\
10 \\
8.4 \\
24 \\
20\end{array}$ & $\begin{array}{l}6.0 \\
9.6 \\
8.6 \\
7.4 \\
6.7\end{array}$ & $\begin{array}{l}5.2 \\
5.2 \\
5.8 \\
5.2 \\
5.0\end{array}$ & $\begin{array}{l}5.3 \\
4.7 \\
5.3 \\
8.6 \\
6.0\end{array}$ & $\begin{array}{l}4.7 \\
4.7 \\
6.7 \\
14 \\
11\end{array}$ & $\begin{array}{l}3.9 \\
3.8 \\
3.8 \\
3.7 \\
3.7\end{array}$ & $\begin{array}{l}11 \\
15 \\
6.5 \\
4.2 \\
3.5\end{array}$ & $\begin{array}{l}4.3 \\
3.4 \\
4.9 \\
4.1 \\
4.7\end{array}$ & $\begin{array}{l}3.7 \\
4.3 \\
3.4 \\
3.1 \\
3.1\end{array}$ \\
\hline $\begin{array}{l}21 \\
22 \\
23 \\
24 \\
25\end{array}$ & $\begin{array}{l}4.5 \\
4.5 \\
4.4 \\
4.3 \\
5.1\end{array}$ & $\begin{array}{r}4.6 \\
4.8 \\
5.7 \\
11 \\
6.5\end{array}$ & $\begin{array}{l}6.3 \\
4.7 \\
4.4 \\
4.3 \\
5.7\end{array}$ & $\begin{array}{r}8.4 \\
7.0 \\
6.7 \\
6.8 \\
30\end{array}$ & $\begin{array}{l}6.3 \\
6.3 \\
6.2 \\
6.3 \\
6.2\end{array}$ & $\begin{array}{c}4.9 \\
4.9 \\
4.9 \\
11 \\
9.1\end{array}$ & $\begin{array}{l}5.5 \\
5.4 \\
5.1 \\
4.8 \\
6.9\end{array}$ & $\begin{array}{l}7.5 \\
5.6 \\
5.0 \\
4.7 \\
4.6\end{array}$ & $\begin{array}{l}3.9 \\
3.8 \\
3.8 \\
3.7 \\
3.7\end{array}$ & $\begin{array}{l}3.2 \\
3.0 \\
3.0 \\
2.8 \\
2.8\end{array}$ & $\begin{array}{l}5.2 \\
4.7 \\
4.2 \\
4.0 \\
3.8\end{array}$ & $\begin{array}{l}3.0 \\
3.0 \\
3.0 \\
3.0 \\
3.0\end{array}$ \\
\hline $\begin{array}{l}26 \\
27 \\
28 \\
29 \\
30 \\
31\end{array}$ & $\begin{array}{l}10 \\
9.8 \\
8.7 \\
6.7 \\
5.9 \\
5.2\end{array}$ & $\begin{array}{r}5.5 \\
5.2 \\
8.1 \\
12 \\
8.0 \\
0-\end{array}$ & $\begin{array}{l}4.9 \\
4.3 \\
5.1 \\
6.6 \\
9.5 \\
9.5\end{array}$ & $\begin{array}{l}27 \\
14 \\
10 \\
9.4 \\
8.7 \\
8.7\end{array}$ & $\begin{array}{l}5.9 \\
5.7 \\
6.3 \\
-.- \\
-\end{array}$ & $\begin{array}{l}12 \\
6.8 \\
5.8 \\
5.7 \\
5.5 \\
5.4\end{array}$ & $\begin{array}{l}9.1 \\
6.8 \\
5.5 \\
5.1 \\
5.0 \\
-.-\end{array}$ & $\begin{array}{l}4.3 \\
6.5 \\
5.1 \\
4.6 \\
4.4 \\
4.2\end{array}$ & $\begin{array}{l}3.8 \\
3.6 \\
3.5 \\
3.4 \\
3.5 \\
-.-\end{array}$ & $\begin{array}{l}3.0 \\
2.9 \\
2.8 \\
2.7 \\
{\left[\begin{array}{l}2.6 \\
2.4\end{array}\right]}\end{array}$ & $\begin{array}{l}3.9 \\
4.0 \\
4.1 \\
4.2 \\
4.3 \\
4.3\end{array}$ & $\begin{array}{l}3.0 \\
3.0 \\
3.0 \\
3.0 \\
4.2 \\
-.0\end{array}$ \\
\hline $\begin{array}{l}\text { TOTAL } \\
\text { MFAN } \\
\text { MAX } \\
\text { MIN }\end{array}$ & $\begin{array}{r}207.0 \\
6.68 \\
13 \\
4.3\end{array}$ & $\begin{array}{r}173.2 \\
5.77 \\
12 \\
4.5\end{array}$ & $\begin{array}{r}227.6 \\
7.34 \\
25 \\
4.3\end{array}$ & $\begin{array}{r}370.4 \\
11.9 \\
34 \\
4.5\end{array}$ & $\begin{array}{r}230.8 \\
8.24 \\
21 \\
5.7\end{array}$ & $\begin{array}{r}219.1 \\
7.07 \\
18 \\
4.9\end{array}$ & $\begin{array}{r}180.3 \\
6.01 \\
16 \\
4.7\end{array}$ & $\begin{array}{r}227.5 \\
7.34 \\
25 \\
4.2\end{array}$ & $\begin{array}{r}151.6 \\
5.05 \\
16 \\
3.4\end{array}$ & $\begin{array}{r}120.1 \\
3.87 \\
15 \\
2.4\end{array}$ & $\begin{array}{r}117.4 \\
3.79 \\
5.2 \\
2.4\end{array}$ & $\begin{array}{r}125.1 \\
4.17 \\
9.5 \\
3.0\end{array}$ \\
\hline
\end{tabular}


UNITED STATES DEPARTMENT OF INTERIOR - GEOLOGICAL SURVEY

PROCESS DATE IS $10-03-83$

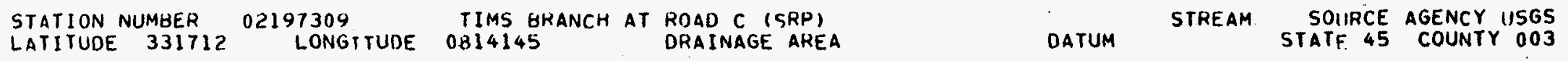

PROVISIONAL DATA

DISCHARGE. IN CUBIC FEET PER SECOND, WATER YEAR OCTOBER 1978 TO SEPTEMBER 1979 MEAN VALUES

\begin{tabular}{|c|c|c|c|c|c|c|c|c|c|c|c|c|}
\hline OAY & OCT & NOV & DEC & JAN & FEB & MAR & APR & MAY & JUN & JUL & AUG & SEP \\
\hline $\begin{array}{l}1 \\
2 \\
3 \\
4 \\
5\end{array}$ & $\begin{array}{l}5.2 \\
4.1 \\
3.5 \\
3.4 \\
3.1\end{array}$ & $\begin{array}{l}2.8 \\
2.8 \\
2.8 \\
2.8 \\
2.8\end{array}$ & $\begin{array}{l}7.2 \\
5.8 \\
5.2 \\
5.0 \\
4.8\end{array}$ & $\begin{array}{l}5.5 \\
8.2 \\
6.9 \\
5.9 \\
5.6\end{array}$ & $\begin{array}{l}5.1 \\
4.7 \\
4.7 \\
5.4 \\
4.8\end{array}$ & $\begin{array}{l}7.9 \\
7.0 \\
5.8 \\
5.5 \\
5.2\end{array}$ & $\begin{array}{l}-.0 \\
4.0 \\
9.6 \\
7.4\end{array}$ & $\begin{array}{l}6.7 \\
6.9 \\
5.6 \\
4.7 \\
5.1\end{array}$ & $\begin{array}{l}25 \\
12 \\
7.6 \\
6.0 \\
5.3\end{array}$ & $\begin{array}{l}5.4 \\
3.2 \\
2.9 \\
3.2 \\
3.1\end{array}$ & $\begin{array}{l}3.3 \\
3.2 \\
2.8 \\
3.2 \\
3.6\end{array}$ & 20 \\
\hline $\begin{array}{r}6 \\
7 \\
8 \\
9 \\
10\end{array}$ & $\begin{array}{l}3.0 \\
2.8 \\
2.8 \\
2.8 \\
2.8\end{array}$ & $\begin{array}{l}2.8 \\
3.0 \\
3.8 \\
3.6 \\
3.4\end{array}$ & $\begin{array}{l}4.3 \\
4.1 \\
4.1 \\
4.3 \\
4.3\end{array}$ & $\begin{array}{l}5.3 \\
5.4 \\
5.5 \\
4.5 \\
4.3\end{array}$ & $\begin{array}{c}8.3 \\
22 \\
9.5 \\
7.1 \\
5.7\end{array}$ & $\begin{array}{l}4.4 \\
3.8 \\
-0 . \\
3.8 \\
3.9\end{array}$ & $\begin{array}{c}5.2 \\
4.5 \\
4.5 \\
10 \\
5.9\end{array}$ & $\begin{array}{l}5.3 \\
4.5 \\
5.8 \\
7.6 \\
6.7\end{array}$ & $\begin{array}{l}5.0 \\
4.9 \\
4.6 \\
4.9 \\
4.6\end{array}$ & $\begin{array}{l}3.0 \\
3.5 \\
4.5 \\
4.0 \\
3.3\end{array}$ & $\begin{array}{l}3.1 \\
2.9 \\
3.0 \\
2.9 \\
2.7\end{array}$ & $\begin{array}{l}13 \\
7.6 \\
4.9 \\
3.8 \\
3.4\end{array}$ \\
\hline $\begin{array}{l}11 \\
12 \\
13 \\
14 \\
15\end{array}$ & $\begin{array}{l}2.7 \\
2.8 \\
2.8 \\
3.1 \\
3.0\end{array}$ & $\begin{array}{l}3.3 \\
3.4 \\
3.3 \\
3.2 \\
3.4\end{array}$ & $\begin{array}{l}4.3 \\
4.9 \\
5.1 \\
4.8 \\
4.4\end{array}$ & $\begin{array}{l}4.3 \\
4.9 \\
5.5 \\
4.9 \\
4.3\end{array}$ & $\begin{array}{l}5.3 \\
5.0 \\
4.8 \\
4.7 \\
4.7\end{array}$ & $\begin{array}{l}5.4 \\
4.2 \\
4.0 \\
4.7 \\
5.0\end{array}$ & $\begin{array}{r}5.5 \\
8.1 \\
12 \\
15 \\
6.8\end{array}$ & $\begin{array}{l}7.1 \\
8.4 \\
14 \\
18 \\
11\end{array}$ & $\begin{array}{l}4.0 \\
3.7 \\
3.6 \\
3.4 \\
3.3\end{array}$ & $\begin{array}{l}3.0 \\
2.9 \\
2.7 \\
2.5 \\
2.4\end{array}$ & $\begin{array}{l}3.2 \\
5.4 \\
3.5 \\
3.2 \\
2.9\end{array}$ & $\begin{array}{l}3.3 \\
3.3 \\
3.4 \\
3.6 \\
3.7\end{array}$ \\
\hline $\begin{array}{l}16 \\
17 \\
18 \\
19 \\
20\end{array}$ & $\begin{array}{l}2.9 \\
2.8 \\
2.8 \\
2.8 \\
2.8\end{array}$ & $\begin{array}{l}3.5 \\
3.5 \\
3.8 \\
3.8 \\
3.5\end{array}$ & $\begin{array}{l}4.4 \\
4.5 \\
4.3 \\
4.3 \\
4.3\end{array}$ & $\begin{array}{l}4.2 \\
4.3 \\
5.0 \\
4.5 \\
4.7\end{array}$ & $\begin{array}{l}4.6 \\
4.3 \\
6.7 \\
6.0 \\
6.5\end{array}$ & $\begin{array}{l}4.3 \\
4.5 \\
4.3 \\
4.3 \\
4.4\end{array}$ & $\begin{array}{l}5.8 \\
6.7 \\
5.8 \\
4.9 \\
4.5\end{array}$ & $\begin{array}{l}7.8 \\
6.2 \\
5.1 \\
4.5 \\
4.6\end{array}$ & $\begin{array}{l}5.1 \\
--. \\
3.8 \\
3.4 \\
3.2\end{array}$ & $\begin{array}{l}2.3 \\
2.6 \\
3.4 \\
3.4 \\
3.1\end{array}$ & $\begin{array}{l}2.8 \\
2.9 \\
2.6 \\
2.6 \\
--.\end{array}$ & $\begin{array}{l}3.1 \\
3.2 \\
3.3 \\
3.2 \\
3.1\end{array}$ \\
\hline $\begin{array}{l}21 \\
22 \\
23 \\
24 \\
25\end{array}$ & $\begin{array}{l}2.8 \\
2.8 \\
2.8 \\
2.8 \\
2.8\end{array}$ & $\begin{array}{l}3.5 \\
3.5 \\
3.5 \\
3.5 \\
3.5\end{array}$ & $\begin{array}{l}4.5 \\
4.9 \\
4.5 \\
8.9 \\
7.6\end{array}$ & $\begin{array}{l}6.2 \\
4.9 \\
4.6 \\
7.9 \\
5.7\end{array}$ & $\begin{array}{l}19 \\
17 \\
-0- \\
-0\end{array}$ & $\begin{array}{l}4.4 \\
5.0 \\
15 \\
17 \\
6.5\end{array}$ & $\begin{array}{l}4.4 \\
4.3 \\
4.2 \\
4.2 \\
15\end{array}$ & $\begin{array}{l}5.4 \\
6.9 \\
8.7 \\
6.6 \\
6.1\end{array}$ & $\begin{array}{l}3.1 \\
5.0 \\
3.4 \\
3.0 \\
3.0\end{array}$ & $\begin{array}{r}6.1 \\
14 \\
8.7 \\
8.3\end{array}$ & $=-$ & $\begin{array}{l}3.5 \\
3.4 \\
3.2 \\
3.2 \\
4.5\end{array}$ \\
\hline $\begin{array}{l}26 \\
27 \\
28 \\
29 \\
30 \\
31\end{array}$ & $\begin{array}{l}2.8 \\
2.9 \\
2.9 \\
3.0 \\
2.8 \\
2.8\end{array}$ & $\begin{array}{l}3.5 \\
4.1 \\
4.6 \\
12 \\
21 \\
-\end{array}$ & $\begin{array}{l}5.0 \\
4.7 \\
4.6 \\
4.6 \\
5.2 \\
6.0\end{array}$ & $\begin{array}{c}5.1 \\
13 \\
6.2 \\
4.7 \\
4.5 \\
5.3\end{array}$ & $\begin{array}{c}19 \\
12 \\
9.2 \\
0-\end{array}$ & $\begin{array}{l}7.9 \\
5.7 \\
5.5 \\
5.2 \\
-.-\end{array}$ & $\begin{array}{l}24 \\
10 \\
7.0 \\
5.7 \\
4.9 \\
-\end{array}$ & $\begin{array}{l}6.7 \\
4.5 \\
4.2 \\
4.2 \\
8.0 \\
11\end{array}$ & $\begin{array}{l}5.2 \\
4.5 \\
3.1 \\
2.7 \\
4.4 \\
-. .-\end{array}$ & $\begin{array}{l}7.6 \\
4.5 \\
3.8 \\
3.5 \\
3.2 \\
3.1\end{array}$ & $\begin{array}{l}=-\infty \\
-\infty \\
-\infty \\
-\infty \\
-\infty\end{array}$ & $\begin{array}{l}4.2 \\
3.9 \\
6.3 \\
7.6 \\
8.9 \\
---\end{array}$ \\
\hline $\begin{array}{l}\text { TOTAL } \\
\text { MEAN } \\
\text { MAX } \\
\text { MIN }\end{array}$ & $\begin{array}{r}93.2 \\
3.01 \\
5.2 \\
2.7\end{array}$ & $\begin{array}{r}128.0 \\
4.27 \\
21 \\
2.8\end{array}$ & $\begin{array}{r}154.9 \\
5.00 \\
8.9 \\
4.1\end{array}$ & $\begin{array}{r}171.8 \\
5.54 \\
13 \\
4.2\end{array}$ & $\begin{array}{l}-- \\
-- \\
--\end{array}$ & $\overline{-m}$ & $=-$ & $\begin{array}{r}217.9 \\
7.03 \\
18 \\
4.2\end{array}$ & $\overline{--}$ & $\begin{array}{l}-\infty \\
-\infty \\
-\infty \\
-\infty\end{array}$ & $\ddot{-\infty}$ & $=$ \\
\hline
\end{tabular}

CAL YR 1978 TOTAL 2118.4 MEAN 5.80 MAX 34 MIN 2.4 
UNITEO STATES DEPARTMENT OF INTERIOR - GEOLOGICAL SURVEY

PROCESS DATE IS 10-03-83

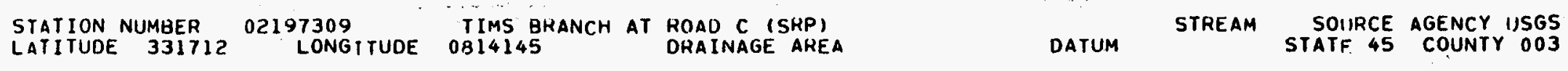
PHOVISIONAL DATA OISCHARGE, IN CUBIC FEET PER SECOND, WATER YEAR OCTOBER 1979 TO SEPTEMBER 1980 MEAN VALUES

\begin{tabular}{|c|c|c|c|c|c|c|c|c|c|c|c|c|}
\hline DAY & OCT & NOV & DEC & JAN & FEA & MAR & APR & MAY & JUN & JUL & AUG & SEP \\
\hline $\begin{array}{l}1 \\
2 \\
3 \\
4 \\
5\end{array}$ & $\begin{array}{l}5.5 \\
4.2 \\
3.4 \\
3.8 \\
6.8\end{array}$ & $\begin{array}{r}3.1 \\
7.2 \\
10 \\
5.3 \\
4.5\end{array}$ & $\begin{array}{l}3.9 \\
4.4 \\
3.6 \\
3.2 \\
4.0\end{array}$ & $\begin{array}{l}4.6 \\
7.1 \\
5.8 \\
3.9 \\
4.4\end{array}$ & $\begin{array}{l}7.3 \\
5.0 \\
4.2 \\
3.8 \\
3.5\end{array}$ & $\begin{array}{l}6.7 \\
7.2 \\
7.6 \\
7.4 \\
7.9\end{array}$ & $\begin{array}{l}12 \\
9.2 \\
8.8 \\
11 \\
12\end{array}$ & $\begin{array}{l}6.4 \\
6.4 \\
6.3 \\
6.2 \\
6.2\end{array}$ & $\begin{array}{l}3.1 \\
2.8 \\
2.7 \\
2.6 \\
2.5\end{array}$ & $\begin{array}{l}2.5 \\
2.6 \\
2.4 \\
5.7 \\
3.1\end{array}$ & $\begin{array}{l}2.1 \\
2.3 \\
3.4 \\
3.1 \\
2.2\end{array}$ & $\begin{array}{r}1.7 \\
2.5 \\
2.3 \\
1.6 \\
1.4\end{array}$ \\
\hline $\begin{array}{r}6 \\
7 \\
8 \\
9 \\
10\end{array}$ & $\begin{array}{l}4.6 \\
3.7 \\
3.4 \\
8.4 \\
5.9\end{array}$ & $\begin{array}{l}4.0 \\
0.2 \\
6.3 \\
4.1 \\
3.9\end{array}$ & $\begin{array}{l}4.4 \\
3.8 \\
3.5 \\
3.9 \\
4.9\end{array}$ & $\begin{array}{l}3.5 \\
3.4 \\
3.6 \\
3.6 \\
3.1\end{array}$ & $\begin{array}{l}4.3 \\
4.7 \\
4.5 \\
6.8 \\
13\end{array}$ & $\begin{array}{l}8.6 \\
6.9 \\
14 \\
14 \\
10\end{array}$ & $\begin{array}{l}8.9 \\
8.0 \\
8.7 \\
13 \\
15\end{array}$ & $\begin{array}{l}6.2 \\
6.0 \\
6.4 \\
7.1 \\
5.9\end{array}$ & $\begin{array}{l}2.6 \\
2.6 \\
2.5 \\
2.4 \\
2.4\end{array}$ & $\begin{array}{l}2.6 \\
3.1 \\
2.7 \\
2.5 \\
2.4\end{array}$ & $\begin{array}{l}3.4 \\
2.9 \\
1.8 \\
2.4 \\
2.0\end{array}$ & $\begin{array}{l}1.4 \\
1.4 \\
1.4 \\
1.4 \\
1.8 \\
\end{array}$ \\
\hline $\begin{array}{l}11 \\
12 \\
13 \\
14 \\
15\end{array}$ & $\begin{array}{l}3.8 \\
3.2 \\
3.2 \\
3.1 \\
2.8\end{array}$ & $\begin{array}{c}19 \\
14 \\
8.3 \\
6.3 \\
5.3\end{array}$ & $\begin{array}{l}4.3 \\
3.5 \\
3.4 \\
3.4 \\
3.7\end{array}$ & $\begin{array}{l}3.6 \\
3.6 \\
3.5 \\
6.8 \\
6.2\end{array}$ & $\begin{array}{l}7.6 \\
6.5 \\
5.9 \\
7.9 \\
6.3\end{array}$ & $\begin{array}{l}15 \\
20 \\
37 \\
19 \\
17\end{array}$ & $\begin{array}{c}11 \\
7.8 \\
8.0 \\
8.0 \\
10\end{array}$ & $\begin{array}{l}5.4 \\
5.2 \\
4.8 \\
5.0 \\
5.0\end{array}$ & $\begin{array}{l}2.4 \\
2.4 \\
2.4 \\
2.3 \\
2.2\end{array}$ & $\begin{array}{l}2.1 \\
2.0 \\
2.0 \\
1.9 \\
2.0\end{array}$ & $\begin{array}{l}1.7 \\
3.9 \\
2.6 \\
2.6 \\
2.0\end{array}$ & $\begin{array}{l}2.5 \\
2.6 \\
3.4 \\
4.6 \\
5.0\end{array}$ \\
\hline $\begin{array}{l}16 \\
17 \\
18 \\
19 \\
20\end{array}$ & $\begin{array}{l}3.4 \\
4.1 \\
3.7 \\
3.8 \\
4.0\end{array}$ & $\begin{array}{l}5.0 \\
7.1 \\
5.8 \\
4.5 \\
3.9\end{array}$ & $\begin{array}{l}3.8 \\
3.8 \\
3.8 \\
3.7 \\
3.5\end{array}$ & $\begin{array}{l}4.9 \\
4.6 \\
6.6 \\
5.7 \\
4.4\end{array}$ & $\begin{array}{l}8.7 \\
7.2 \\
6.0 \\
5.8 \\
6.1\end{array}$ & $\begin{array}{c}10 \\
9.9 \\
18 \\
9.6 \\
12\end{array}$ & $\begin{array}{l}8.6 \\
7.8 \\
7.4 \\
7.3 \\
7.4\end{array}$ & $\begin{array}{l}4.7 \\
5.0 \\
5.1 \\
4.5 \\
6.3\end{array}$ & $\begin{array}{l}2.0 \\
2.2 \\
6.4 \\
7.9 \\
4.5\end{array}$ & $\begin{array}{l}1.9 \\
1.9 \\
1.8 \\
1.9 \\
1.8 \quad 1\end{array}$ & $\begin{array}{l}1.7 \\
1.5 \\
1.7 \\
1.9 \\
4.9\end{array}$ & $\begin{array}{l}5.3 \\
6.5 \\
3.6 \\
1.6 \\
1.7\end{array}$ \\
\hline $\begin{array}{l}21 \\
22 \\
23 \\
24 \\
25\end{array}$ & $\begin{array}{l}3.6 \\
3.8 \\
4.2 \\
3.8 \\
3.7\end{array}$ & $\begin{array}{l}3.9 \\
3.7 \\
3.6 \\
3.7 \\
3.5\end{array}$ & $\begin{array}{l}4.0 \\
3.6 \\
4.9 \\
4.5 \\
3.7\end{array}$ & $\begin{array}{l}4.2 \\
4.4 \\
8.6 \\
5.7 \\
5.0\end{array}$ & $\begin{array}{l}10 \\
9.1 \\
8.4 \\
7.0 \\
6.3\end{array}$ & $\begin{array}{l}13 \\
13 \\
8.9 \\
11 \\
21\end{array}$ & $\begin{array}{l}7.1 \\
7.0 \\
6.8 \\
6.7 \\
6.6\end{array}$ & $\begin{array}{l}6.1 \\
8.2 \\
10 \\
16 \\
7.8\end{array}$ & $\begin{array}{l}3.6 \\
3.3 \\
3.1 \\
3.3 \\
5.3\end{array}$ & $\begin{array}{l}1.8 \\
1.8 \\
1.8 \\
2.3 \\
2.3\end{array}$ & $\begin{array}{l}3.1 \\
2.5 \\
2.2 \\
2.2 \\
1.9\end{array}$ & $\begin{array}{l}1.8 \\
2.6 \\
2.2 \\
2.9 \\
8.1\end{array}$ \\
\hline $\begin{array}{l}26 \\
27 \\
28 \\
29 \\
30 \\
31\end{array}$ & $\begin{array}{l}3.8 \\
3.9 \\
3.7 \\
3.7 \\
3.8 \\
3.8\end{array}$ & $\begin{array}{l}3.6 \\
3.5 \\
4.2 \\
6.0 \\
5.4 \\
-0 .-\end{array}$ & $\begin{array}{l}3.4 \\
3.5 \\
3.9 \\
7.9 \\
6.9 \\
5.5\end{array}$ & $\begin{array}{l}6.1 \\
10 \\
6.2 \\
5.0 \\
4.2 \\
11\end{array}$ & $\begin{array}{l}8.3 \\
7.7 \\
6.7 \\
6.4 \\
--. \\
---\end{array}$ & $\begin{array}{l}26 \\
8.7 \\
21 \\
26 \\
26 \\
17\end{array}$ & $\begin{array}{l}6.5 \\
6.5 \\
6.4 \\
6.4 \\
6.4 \\
-.-\end{array}$ & $\begin{array}{l}5.5 \\
4.3 \\
3.7 \\
4.6 \\
4.1 \\
3.5\end{array}$ & $\begin{array}{l}6.8 \\
4.4 \\
3.5 \\
3.1 \\
2.6 \\
.-0\end{array}$ & $\begin{array}{l}2.2 \\
2.1 \\
2.5 \\
2.7 \\
2.3 \\
2.1\end{array}$ & $\begin{array}{l}1.6 \\
1.6 \\
1.8 \\
1.6 \\
1.5 \\
1.7\end{array}$ & $\begin{array}{c}8.2 \\
5.8 \\
7.6 \\
20 \\
17 \\
-\end{array}$ \\
\hline $\begin{array}{l}\text { TOTAL } \\
\text { MEAN } \\
\text { MAX } \\
\text { MIN }\end{array}$ & $\begin{array}{r}126.6 \\
4.08 \\
8.4 \\
2.8\end{array}$ & $\begin{array}{r}176.9 \\
5.90 \\
19 \\
3.1\end{array}$ & $\begin{array}{r}128.3 \\
4.14 \\
7.9 \\
3.2\end{array}$ & $\begin{array}{r}163.3 \\
5.27 \\
11 \\
3.1\end{array}$ & $\begin{array}{r}195.0 \\
6.72 \\
13 \\
3.5\end{array}$ & $\begin{array}{r}449.4 \\
14.5 \\
37 \\
6.7\end{array}$ & $\begin{array}{r}257.1 \\
8.57 \\
15 \\
6.4\end{array}$ & $\begin{array}{r}187.9 \\
6.06 \\
16 \\
3.5\end{array}$ & $\begin{array}{r}99.9 \\
3.33 \\
7.9 \\
2.0\end{array}$ & $\begin{array}{r}72.8 \\
2.35 \\
5.7 \\
1.8\end{array}$ & $\begin{array}{r}71.8 \\
2.32 \\
4.9 \\
1.5\end{array}$ & $\begin{array}{r}129.9 \\
4.33 \\
20 \\
1.4\end{array}$ \\
\hline
\end{tabular}


UNITED STATES DEPARTMENT OF INTERIOR - GEOLOGICAL SURVEY

PROCESS DATE IS 10-03-83

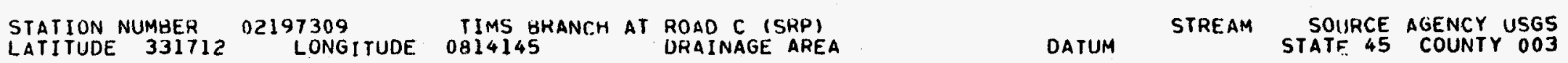

PROVISIONAL DATA

DISCHARGE, IN CUBIC FEET PER SECOND, WATER YEAR OCTOBER 1980 TO SEPTEMBER I9RI MEAN VALUES

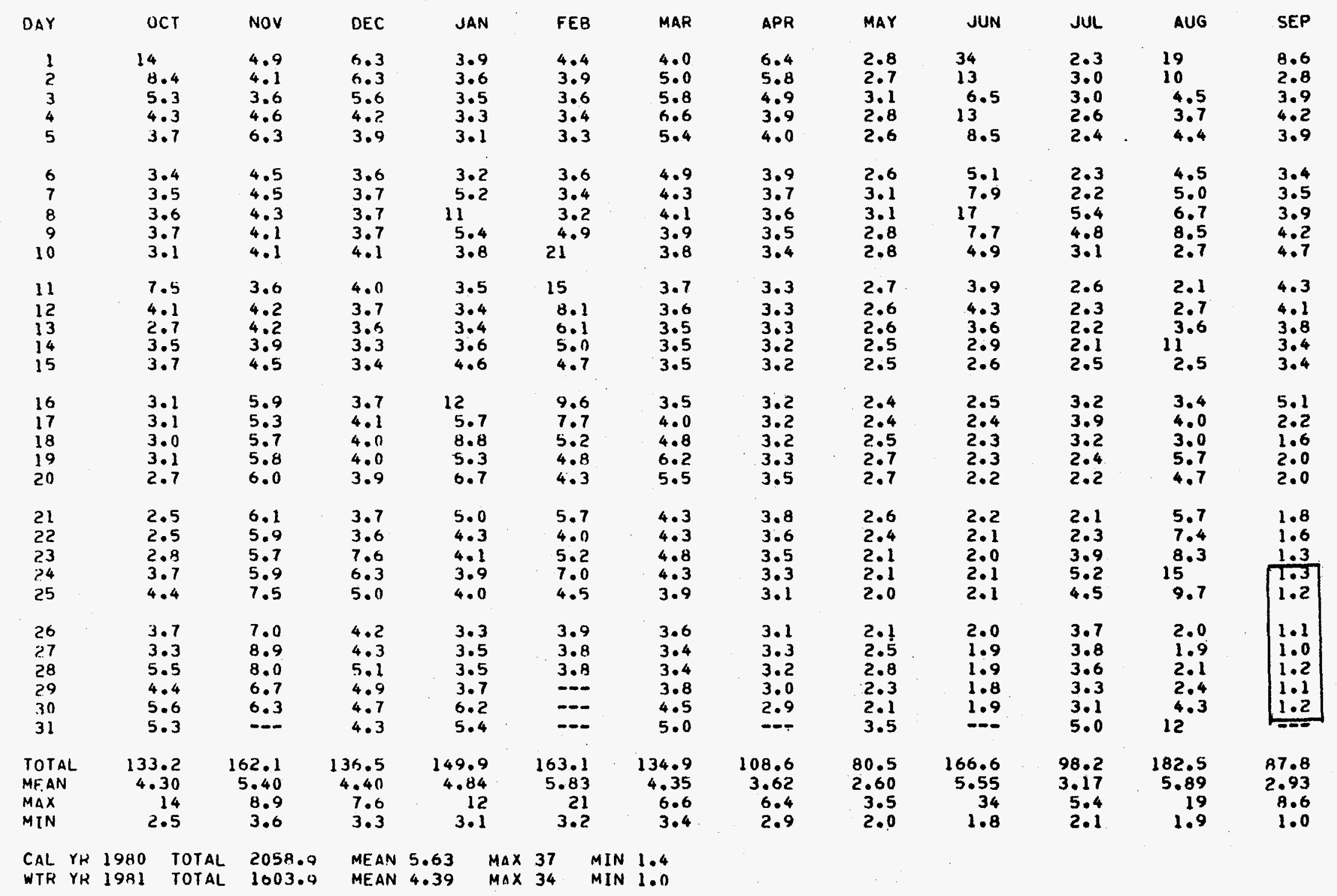




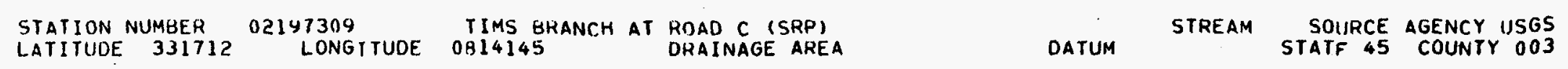

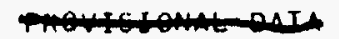

WTR YR 1992 TOTAL

\begin{tabular}{|c|c|c|c|c|c|c|c|c|c|c|c|c|}
\hline DAY & OCT & NoV & DEC & JAN & FEB & MAR & $A P R$ & MAY & JUN & JUL & AUG & SEP \\
\hline $\begin{array}{l}1 \\
2 \\
3 \\
4 \\
5\end{array}$ & $\begin{array}{l}1.4 \\
1.4 \\
1.3 \\
1.3 \\
1.7\end{array}$ & $\begin{array}{l}2.3 \\
2.1 \\
2.3 \\
2.3 \\
1.9\end{array}$ & $\begin{array}{l}6.3 \\
9.3 \\
7.9 \\
8.3 \\
8.3\end{array}$ & $\begin{array}{l}30 \\
16 \\
18 \\
18 \\
14\end{array}$ & $\begin{array}{l}4.3 \\
3.5 \\
6.8 \\
9.2 \\
6.0\end{array}$ & $\begin{array}{l}3.5 \\
3.1 \\
3.9 \\
3.8 \\
3.7\end{array}$ & $\begin{array}{l}3.1 \\
3.0 \\
3.0 \\
2.9 \\
3.9\end{array}$ & $\begin{array}{l}4.9 \\
4.6 \\
4.4 \\
6.3 \\
5.7\end{array}$ & $\begin{array}{l}2.6 \\
2.3 \\
2.7 \\
4.5 \\
3.9\end{array}$ & $\begin{array}{l}2.2 \\
2.1 \\
1.9 \\
1.8 \\
1.9\end{array}$ & $\begin{array}{l}5.9 \\
5.1 \\
4.8 \\
3.5 \\
5.6\end{array}$ & $\begin{array}{l}1.9 \\
1.9 \\
1.8 \\
1.7 \\
1.6\end{array}$ \\
\hline $\begin{array}{r}6 \\
7 \\
8 \\
9 \\
10\end{array}$ & $\begin{array}{l}1.7 \\
1.5 \\
1.6 \\
1.6 \\
3.3\end{array}$ & $\begin{array}{l}4.4 \\
5.2 \\
3.2 \\
2.5 \\
2.4\end{array}$ & $\begin{array}{l}7.5 \\
7.4 \\
7.3 \\
7.7 \\
8.1\end{array}$ & $\begin{array}{l}10 \\
9.2 \\
8.8 \\
8.3 \\
7.8\end{array}$ & $\begin{array}{l}4.8 \\
4.0 \\
3.7 \\
4.0 \\
5.0\end{array}$ & $\begin{array}{l}3.5 \\
7.8 \\
6.5 \\
4.0 \\
4.4\end{array}$ & $\begin{array}{l}4.0 \\
3.3 \\
3.9 \\
4.9 \\
3.9\end{array}$ & $\begin{array}{l}5.1 \\
4.5 \\
4.0 \\
3.7 \\
3.4\end{array}$ & $\begin{array}{l}3.1 \\
2.6 \\
2.3 \\
2.2 \\
2.1\end{array}$ & $\begin{array}{l}1.9 \\
1.9 \\
1.9 \\
2.0 \\
2.7\end{array}$ & $\begin{array}{l}10 \\
6.0 \\
4.3 \\
4.3 \\
3.5\end{array}$ & $\begin{array}{l}1.4 \\
1.4 \\
1.3 \\
1.3 \\
1.5\end{array}$ \\
\hline $\begin{array}{l}11 \\
12 \\
13 \\
14 \\
15\end{array}$ & $\begin{array}{l}3.1 \\
2.5 \\
2.2 \\
2.0 \\
2.0\end{array}$ & $\begin{array}{l}2.6 \\
2.7 \\
2.5 \\
2.3 \\
2.3\end{array}$ & $\begin{array}{l}8.6 \\
8.6 \\
8.7 \\
12 \\
21\end{array}$ & $\begin{array}{l}5.0 \\
3.4 \\
4.1 \\
7.9 \\
7.6\end{array}$ & $\begin{array}{l}5.2 \\
5.5 \\
8.6 \\
5.2 \\
3.7\end{array}$ & $\begin{array}{l}4.2 \\
4.1 \\
3.6 \\
3.1 \\
3.0\end{array}$ & $\begin{array}{l}3.4 \\
3.2 \\
2.9 \\
2.8 \\
3.4\end{array}$ & $\begin{array}{l}3.2 \\
3.2 \\
3.0 \\
3.0 \\
2.9\end{array}$ & $\begin{array}{l}2.1 \\
2.0 \\
2.9 \\
2.8 \\
2.4\end{array}$ & $\begin{array}{l}2.4 \\
2.7 \\
4.8 \\
3.3 \\
2.6\end{array}$ & $\begin{array}{l}2.7 \\
2.7 \\
5.6 \\
3.0 \\
2.3\end{array}$ & $\begin{array}{l}1.8 \\
1.9 \\
1.9 \\
2.0 \\
1.9\end{array}$ \\
\hline $\begin{array}{l}16 \\
17 \\
18 \\
19 \\
20\end{array}$ & $\begin{array}{l}1.9 \\
1.9 \\
1.9 \\
2.0 \\
1.8\end{array}$ & $\begin{array}{l}2.3 \\
2.4 \\
2.3 \\
2.4 \\
2.5\end{array}$ & $\begin{array}{l}18 \\
9.4 \\
5.6 \\
5.1 \\
4.1\end{array}$ & $\begin{array}{l}5.2 \\
4.4 \\
3.8 \\
4.1 \\
4.3\end{array}$ & $\begin{array}{l}3.7 \\
7.6 \\
5.9 \\
5.6 \\
4.7\end{array}$ & $\begin{array}{l}3.5 \\
6.7 \\
7.3 \\
5.1 \\
4.4\end{array}$ & $\begin{array}{l}3.5 \\
4.2 \\
4.5 \\
3.3 \\
7.2\end{array}$ & $\begin{array}{l}2.7 \\
2.6 \\
5.8 \\
4.4 \\
3.5\end{array}$ & $\begin{array}{l}2.3 \\
3.1 \\
2.9 \\
2.5 \\
2.2\end{array}$ & $\begin{array}{l}2.5 \\
2.6 \\
2.5 \\
2.1 \\
2.2\end{array}$ & $\begin{array}{l}2.0 \\
2.0 \\
3.4 \\
7.0 \\
3.7\end{array}$ & $\begin{array}{l}1.7 \\
1.5 \\
3.3 \\
12 \\
7.1\end{array}$ \\
\hline $\begin{array}{l}21 \\
22 \\
23 \\
24 \\
25\end{array}$ & $\begin{array}{l}1.9 \\
1.9 \\
1.9 \\
2.1 \\
2.1\end{array}$ & $\begin{array}{l}2.5 \\
2.6 \\
2.6 \\
2.8 \\
2.8\end{array}$ & $\begin{array}{l}3.8 \\
3.9 \\
3.9 \\
3.7 \\
9.1\end{array}$ & $\begin{array}{l}6.0 \\
4.0 \\
4.4 \\
6.5 \\
4.6\end{array}$ & $\begin{array}{l}3.9 \\
3.2 \\
3.2 \\
3.9 \\
4.2\end{array}$ & $\begin{array}{l}4.0 \\
3.4 \\
3.3 \\
3.6 \\
4.3\end{array}$ & $\begin{array}{l}7.7 \\
7.2 \\
6.0 \\
4.7 \\
5.6\end{array}$ & $\begin{array}{l}3.0 \\
2.8 \\
2.8 \\
2.6 \\
2.7\end{array}$ & $\begin{array}{l}2.3 \\
4.2 \\
3.5 \\
4.4 \\
4.6\end{array}$ & $\begin{array}{l}3.5 \\
2.4 \\
2.3 \\
2.7 \\
2.8\end{array}$ & $\begin{array}{l}2.9 \\
2.5 \\
2.4 \\
2.0 \\
1.6\end{array}$ & $\begin{array}{l}6.4 \\
5.6 \\
4.0 \\
3.3 \\
3.0\end{array}$ \\
\hline $\begin{array}{l}26 \\
27 \\
28 \\
29 \\
30 \\
31\end{array}$ & $\begin{array}{l}4.6 \\
3.4 \\
2.9 \\
2.5 \\
2.3 \\
2.3\end{array}$ & $\begin{array}{l}2.9 \\
3.2 \\
3.7 \\
4.4 \\
4.7 \\
-.-\end{array}$ & $\begin{array}{r}7.5 \\
5.5 \\
5.5 \\
8.0 \\
5.5 \\
21\end{array}$ & $\begin{array}{l}3.8 \\
3.5 \\
3.5 \\
3.5 \\
4.8 \\
4.3\end{array}$ & $\begin{array}{l}4.3 \\
4.8 \\
4.1 \\
-.- \\
-.-\end{array}$ & $\begin{array}{l}4.1 \\
3.5 \\
3.2 \\
3.4 \\
3.2 \\
3.2\end{array}$ & $\begin{array}{r}11 \\
14 \\
9.9 \\
7.0 \\
6.0 \\
-\end{array}$ & $\begin{array}{l}2.6 \\
2.5 \\
2.4 \\
2.3 \\
2.6 \\
3.1\end{array}$ & $\begin{array}{l}4.8 \\
7.0 \\
7.2 \\
4.2 \\
3.0 \\
-.-0\end{array}$ & $\begin{array}{r}3.7 \\
3.2 \\
2.6 \\
5.9 \\
12 \\
7.6\end{array}$ & $\begin{array}{l}1.4 \\
1.8 \\
1.7 \\
1.4 \\
1.6 \\
1.7\end{array}$ & $\begin{array}{l}3.0 \\
3.0 \\
2.8 \\
2.7 \\
2.6 \\
-.-\end{array}$ \\
\hline $\begin{array}{l}\text { TOTAL } \\
\text { MFAN } \\
\text { MAX } \\
\text { MIN }\end{array}$ & $\begin{array}{r}66.0 \\
2.13 \\
4.6 \\
1.3\end{array}$ & $\begin{array}{r}85.1 \\
2.84 \\
5.2 \\
1.9\end{array}$ & $\begin{array}{r}256.6 \\
8.28 \\
21 \\
3.7\end{array}$ & $\begin{array}{r}238.8 \\
7.70 \\
30 \\
3.4\end{array}$ & $\begin{array}{r}138.6 \\
4.95 \\
9.2 \\
3.2\end{array}$ & $\begin{array}{r}128.4 \\
4.14 \\
7.8 \\
3.0\end{array}$ & $\begin{array}{r}153.4 \\
5.11 \\
14 \\
2.8\end{array}$ & $\begin{array}{r}110.3 \\
3.56 \\
6.3 \\
2.3\end{array}$ & $\begin{array}{r}98.7 \\
3.29 \\
7.2 \\
2.0\end{array}$ & $\begin{array}{r}96.7 \\
3.12 \\
12 \\
1.8\end{array}$ & $\begin{array}{r}108.4 \\
3.50 \\
10 \\
1.4\end{array}$ & $\begin{array}{r}87.3 \\
2.91 \\
12 \\
1.3\end{array}$ \\
\hline
\end{tabular}

\begin{tabular}{|c|c|c|c|c|c|c|c|c|c|c|c|c|}
\hline DAY & OCT & NoV & DEC & JAN & FEB & MAR & $A P R$ & MAY & JUN & JUL & AUG & SEP \\
\hline $\begin{array}{l}1 \\
2 \\
3 \\
4 \\
5\end{array}$ & $\begin{array}{l}1.4 \\
1.4 \\
1.3 \\
1.3 \\
1.7\end{array}$ & $\begin{array}{l}2.3 \\
2.1 \\
2.3 \\
2.3 \\
1.9\end{array}$ & $\begin{array}{l}6.3 \\
9.3 \\
7.9 \\
8.3 \\
8.3\end{array}$ & $\begin{array}{l}30 \\
16 \\
18 \\
18 \\
14\end{array}$ & $\begin{array}{l}4.3 \\
3.5 \\
6.8 \\
9.2 \\
6.0\end{array}$ & $\begin{array}{l}3.5 \\
3.1 \\
3.9 \\
3.8 \\
3.7\end{array}$ & $\begin{array}{l}3.1 \\
3.0 \\
3.0 \\
2.9 \\
3.9\end{array}$ & $\begin{array}{l}4.9 \\
4.6 \\
4.4 \\
6.3 \\
5.7\end{array}$ & $\begin{array}{l}2.6 \\
2.3 \\
2.7 \\
4.5 \\
3.9\end{array}$ & $\begin{array}{l}2.2 \\
2.1 \\
1.9 \\
1.8 \\
1.9\end{array}$ & $\begin{array}{l}5.9 \\
5.1 \\
4.8 \\
3.5 \\
5.6\end{array}$ & $\begin{array}{l}1.9 \\
1.9 \\
1.8 \\
1.7 \\
1.6\end{array}$ \\
\hline $\begin{array}{r}6 \\
7 \\
8 \\
9 \\
10\end{array}$ & $\begin{array}{l}1.7 \\
1.5 \\
1.6 \\
1.6 \\
3.3\end{array}$ & $\begin{array}{l}4.4 \\
5.2 \\
3.2 \\
2.5 \\
2.4\end{array}$ & $\begin{array}{l}7.5 \\
7.4 \\
7.3 \\
7.7 \\
8.1\end{array}$ & $\begin{array}{l}10 \\
9.2 \\
8.8 \\
8.3 \\
7.8\end{array}$ & $\begin{array}{l}4.8 \\
4.0 \\
3.7 \\
4.0 \\
5.0\end{array}$ & $\begin{array}{l}3.5 \\
7.8 \\
6.5 \\
4.0 \\
4.4\end{array}$ & $\begin{array}{l}4.0 \\
3.3 \\
3.9 \\
4.9 \\
3.9\end{array}$ & $\begin{array}{l}5.1 \\
4.5 \\
4.0 \\
3.7 \\
3.4\end{array}$ & $\begin{array}{l}3.1 \\
2.6 \\
2.3 \\
2.2 \\
2.1\end{array}$ & $\begin{array}{l}1.9 \\
1.9 \\
1.9 \\
2.0 \\
2.7\end{array}$ & $\begin{array}{l}10 \\
6.0 \\
4.3 \\
4.3 \\
3.5\end{array}$ & $\begin{array}{l}1.4 \\
1.4 \\
1.3 \\
1.3 \\
1.5\end{array}$ \\
\hline $\begin{array}{l}11 \\
12 \\
13 \\
14 \\
15\end{array}$ & $\begin{array}{l}3.1 \\
2.5 \\
2.2 \\
2.0 \\
2.0\end{array}$ & $\begin{array}{l}2.6 \\
2.7 \\
2.5 \\
2.3 \\
2.3\end{array}$ & $\begin{array}{l}8.6 \\
8.6 \\
8.7 \\
12 \\
21\end{array}$ & $\begin{array}{l}5.0 \\
3.4 \\
4.1 \\
7.9 \\
7.6\end{array}$ & $\begin{array}{l}5.2 \\
5.5 \\
8.6 \\
5.2 \\
3.7\end{array}$ & $\begin{array}{l}4.2 \\
4.1 \\
3.6 \\
3.1 \\
3.0\end{array}$ & $\begin{array}{l}3.4 \\
3.2 \\
2.9 \\
2.8 \\
3.4\end{array}$ & $\begin{array}{l}3.2 \\
3.2 \\
3.0 \\
3.0 \\
2.9\end{array}$ & $\begin{array}{l}2.1 \\
2.0 \\
2.9 \\
2.8 \\
2.4\end{array}$ & $\begin{array}{l}2.4 \\
2.7 \\
4.8 \\
3.3 \\
2.6\end{array}$ & $\begin{array}{l}2.7 \\
2.7 \\
5.6 \\
3.0 \\
2.3\end{array}$ & $\begin{array}{l}1.8 \\
1.9 \\
1.9 \\
2.0 \\
1.9\end{array}$ \\
\hline $\begin{array}{l}16 \\
17 \\
18 \\
19 \\
20\end{array}$ & $\begin{array}{l}1.9 \\
1.9 \\
1.9 \\
2.0 \\
1.8\end{array}$ & $\begin{array}{l}2.3 \\
2.4 \\
2.3 \\
2.4 \\
2.5\end{array}$ & $\begin{array}{l}18 \\
9.4 \\
5.6 \\
5.1 \\
4.1\end{array}$ & $\begin{array}{l}5.2 \\
4.4 \\
3.8 \\
4.1 \\
4.3\end{array}$ & $\begin{array}{l}3.7 \\
7.6 \\
5.9 \\
5.6 \\
4.7\end{array}$ & $\begin{array}{l}3.5 \\
6.7 \\
7.3 \\
5.1 \\
4.4\end{array}$ & $\begin{array}{l}3.5 \\
4.2 \\
4.5 \\
3.3 \\
7.2\end{array}$ & $\begin{array}{l}2.7 \\
2.6 \\
5.8 \\
4.4 \\
3.5\end{array}$ & $\begin{array}{l}2.3 \\
3.1 \\
2.9 \\
2.5 \\
2.2\end{array}$ & $\begin{array}{l}2.5 \\
2.6 \\
2.5 \\
2.1 \\
2.2\end{array}$ & $\begin{array}{l}2.0 \\
2.0 \\
3.4 \\
7.0 \\
3.7\end{array}$ & $\begin{array}{l}1.7 \\
1.5 \\
3.3 \\
12 \\
7.1\end{array}$ \\
\hline $\begin{array}{l}21 \\
22 \\
23 \\
24 \\
25\end{array}$ & $\begin{array}{l}1.9 \\
1.9 \\
1.9 \\
2.1 \\
2.1\end{array}$ & $\begin{array}{l}2.5 \\
2.6 \\
2.6 \\
2.8 \\
2.8\end{array}$ & $\begin{array}{l}3.8 \\
3.9 \\
3.9 \\
3.7 \\
9.1\end{array}$ & $\begin{array}{l}6.0 \\
4.0 \\
4.4 \\
6.5 \\
4.6\end{array}$ & $\begin{array}{l}3.9 \\
3.2 \\
3.2 \\
3.9 \\
4.2\end{array}$ & $\begin{array}{l}4.0 \\
3.4 \\
3.3 \\
3.6 \\
4.3\end{array}$ & $\begin{array}{l}7.7 \\
7.2 \\
6.0 \\
4.7 \\
5.6\end{array}$ & $\begin{array}{l}3.0 \\
2.8 \\
2.8 \\
2.6 \\
2.7\end{array}$ & $\begin{array}{l}2.3 \\
4.2 \\
3.5 \\
4.4 \\
4.6\end{array}$ & $\begin{array}{l}3.5 \\
2.4 \\
2.3 \\
2.7 \\
2.8\end{array}$ & $\begin{array}{l}2.9 \\
2.5 \\
2.4 \\
2.0 \\
1.6\end{array}$ & $\begin{array}{l}6.4 \\
5.6 \\
4.0 \\
3.3 \\
3.0\end{array}$ \\
\hline $\begin{array}{l}26 \\
27 \\
28 \\
29 \\
30 \\
31\end{array}$ & $\begin{array}{l}4.6 \\
3.4 \\
2.9 \\
2.5 \\
2.3 \\
2.3\end{array}$ & $\begin{array}{l}2.9 \\
3.2 \\
3.7 \\
4.4 \\
4.7 \\
-.-\end{array}$ & $\begin{array}{r}7.5 \\
5.5 \\
5.5 \\
8.0 \\
5.5 \\
21\end{array}$ & $\begin{array}{l}3.8 \\
3.5 \\
3.5 \\
3.5 \\
4.8 \\
4.3\end{array}$ & $\begin{array}{l}4.3 \\
4.8 \\
4.1 \\
-.- \\
-.-\end{array}$ & $\begin{array}{l}4.1 \\
3.5 \\
3.2 \\
3.4 \\
3.2 \\
3.2\end{array}$ & $\begin{array}{r}11 \\
14 \\
9.9 \\
7.0 \\
6.0 \\
-\end{array}$ & $\begin{array}{l}2.6 \\
2.5 \\
2.4 \\
2.3 \\
2.6 \\
3.1\end{array}$ & $\begin{array}{l}4.8 \\
7.0 \\
7.2 \\
4.2 \\
3.0 \\
-.-0\end{array}$ & $\begin{array}{r}3.7 \\
3.2 \\
2.6 \\
5.9 \\
12 \\
7.6\end{array}$ & $\begin{array}{l}1.4 \\
1.8 \\
1.7 \\
1.4 \\
1.6 \\
1.7\end{array}$ & $\begin{array}{l}3.0 \\
3.0 \\
2.8 \\
2.7 \\
2.6 \\
-.-\end{array}$ \\
\hline $\begin{array}{l}\text { TOTAL } \\
\text { MFAN } \\
\text { MAX } \\
\text { MIN }\end{array}$ & $\begin{array}{r}66.0 \\
2.13 \\
4.6 \\
1.3\end{array}$ & $\begin{array}{r}85.1 \\
2.84 \\
5.2 \\
1.9\end{array}$ & $\begin{array}{r}256.6 \\
8.28 \\
21 \\
3.7\end{array}$ & $\begin{array}{r}238.8 \\
7.70 \\
30 \\
3.4\end{array}$ & $\begin{array}{r}138.6 \\
4.95 \\
9.2 \\
3.2\end{array}$ & $\begin{array}{r}128.4 \\
4.14 \\
7.8 \\
3.0\end{array}$ & $\begin{array}{r}153.4 \\
5.11 \\
14 \\
2.8\end{array}$ & $\begin{array}{r}110.3 \\
3.56 \\
6.3 \\
2.3\end{array}$ & $\begin{array}{r}98.7 \\
3.29 \\
7.2 \\
2.0\end{array}$ & $\begin{array}{r}96.7 \\
3.12 \\
12 \\
1.8\end{array}$ & $\begin{array}{r}108.4 \\
3.50 \\
10 \\
1.4\end{array}$ & $\begin{array}{r}87.3 \\
2.91 \\
12 \\
1.3\end{array}$ \\
\hline
\end{tabular}

DISCHARGE, IN CUBIC FFET PER SECOND, WATER YEAR OCTOBER 1981 TO SEPTEMBER 1982 MEAN VALUES 
$\left.\begin{array}{l}7 \\ 4 \\ 4\end{array}\right]$

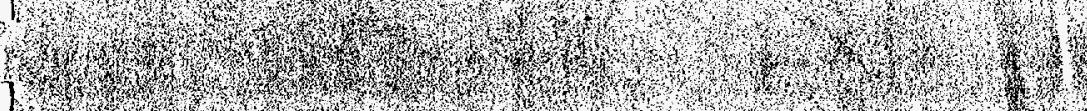

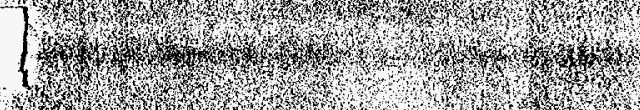

1

APPEND IX 1 - ANGLYTCAL RESULTS

1

]

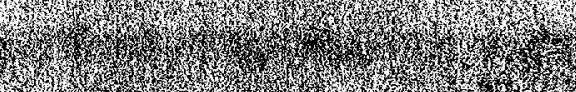

]

1

3

1

]

7

]

列

1

1

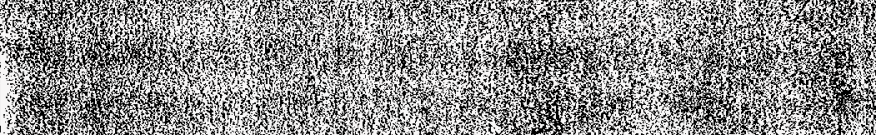

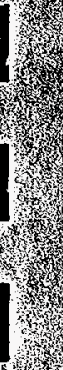

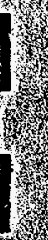

(1)

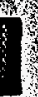

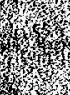

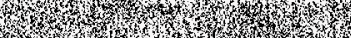

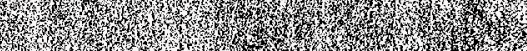

r.t.

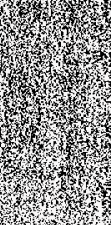

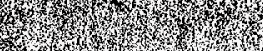


LOCKWOOD GREENE ENG INEERS

LAB NO: 5-274

PO \#25667

October 20,1983

1

]

1

1

1

1 


\section{TABLE OF CONTENTS:}

\section{LABORATORY CHRONICLE}

II. ANALYTICAL METHODS: DEFINITIONS \& EXPLANATIONS

III. RECORDED RESULTS

IV. QUALITY CONTROL DATA

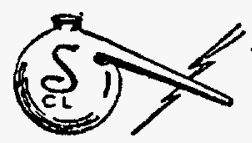




\section{Scientific CONTROL LABORATORIES, INC.}

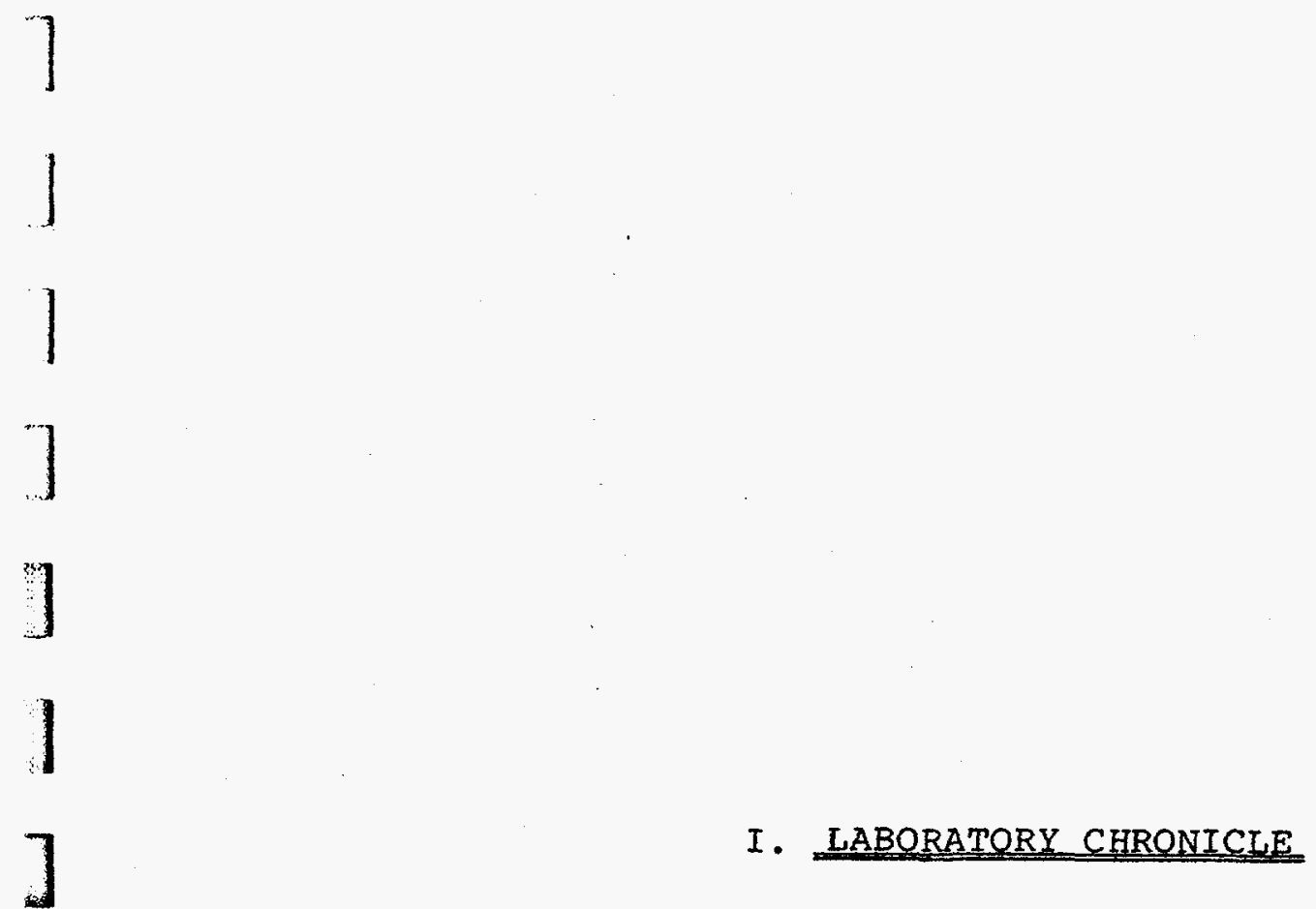

]

1

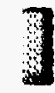

1

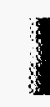

3

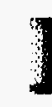

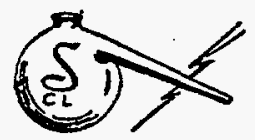


$4: 30 \mathrm{PM}$

$10-10-83$

Samples received and refrigerated. (Note: $\begin{array}{llll}6 & \text { (six) } & 2 & 1 / 2\end{array}$

gallon size jars were received. Of the six sample jars received, Sample 1 consisted of a full container; Sample 2 consisted of two jars, one jar completely full, the second approximately half full. Sample 3 contained approximately 200 to $250 \mathrm{mls}$. Sample 4 contained approximately $500 \mathrm{mls}$. Sample 5 contained approximately $300 \mathrm{mls}$.

$10-11-83$

Customer notified of possible analytical problems arising from insufficient sample. Work to proceed on Samples $1,2 \& 4$. All samples are to remain refrigerated. $\underline{10-11-83}$

Portions of samples $1,2 \& 4$ sent to Mead Compu-Chem for Non Volatile Total Toxic organics testing.

$10-11-83$

Samples identified as 1 thru 5 inclusive sent to Mead Compu-Chem for volatile Toxic organics testing.

$10-13-83$

Instructions from Mr. Nichols regarding order of priority to be followed in testing parameters for samples $3 \& 5$.

$10-20-83$

Formal report completed. 
]

3

3

3

1

1

了

1

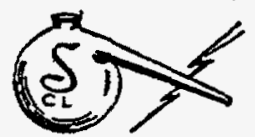


PARAMETER

Sodium

Copper

Lead

Nickel

Iron

Total Phosphorus

Oil \& Grease

$\mathrm{pH}$

Uranium

Total Dissolved Solids

Total Organic Carbon

Aluminum

Nitrate

Total Suspended Solids

Arsenic

Nitrite

Boron

\section{TEST PROCEDURES :}

Flame Emission/AA*, Standard Methods; 15th Edition, pg. 231 Sec. 325A

Digestion/AA*, standard Methods; 15th Edition, pg. 152 Sec. 303A

Digestion/AA*, Standard Methods; 15th Edition, pg. 152 Sec. 303A

Digestion/AA*, Standard Methods; 15th Edition, pg. 152 Sec. 303A

Digestion/AA*, Standard Methods; 15th Edition, pg. 152 Sec. 303A

Digestion/Colorimetric Method, Standard Methods; 15th Edition, pg. 415 Sec. 424D

Partition-Gravimetric Method, Standard Methods; 15th Edition, pg. 461 Sec. 503A

Instrumentation - A Fisher Accumet Model $620 \mathrm{pH}$ Meter

Thiocyanate Method - Scotts' Standard Methods of Chemical Analysis; 6 th Edition, Volume 1

Filtrable Residue, Standard Methods; 15th Edition, pg. 94 Sec. 209C

Combustion-Infrared Method, Standard Methods; 15th Edition, pg. $471 \mathrm{Sec}$. 505

Digestion/AA*, Standard Methods; 15th Edition, pg. 157 Sec. 303C

Cadmium Reduction, Standard Methods; 15th Edition, pg. 370 Sec. $418 \mathrm{C}$

Suspended Matter, Standard Methods; 15th Edition, pg. 94 Sec. 209D

Hydride System, Standard Methods; 15th Edition, pg. 160 sec. $303 \mathrm{E}$ NED dehydrochloride Method, Standard Methods; 15th Edition, pg. $380 \mathrm{Sec} .419$ Curcumin Method, Standard Methods; 15th Edition, pg. 257 Sec. $404 \mathrm{~A}$

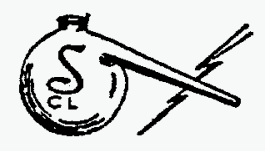


1

1

1

]

3

1

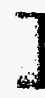

]

(cont.)

PARAMETER

Cadmium

Chromium

Lithium

Phenol

Sulfate

Chloride

Barium

Mercury

Selenium

Silver

\section{TEST PROCEDURES :}

Digestion/AA*, Standard Methods; 15th Edition, pg. 152 Sec. 303A

Digestion/AA*, Standard Methods; 15 th Edition, pg. 152 Sec. 303A

Digestion/AA*, Standard Methods; 15th Edition, pg. 152 Sec. 303A

Chloroform Extraction, Standard Methods; 15th Edition, pg. 510 Sec.510B

Turbidrmetric Method, Standard Methods; 15th Edition, pg. 439 Sec.426C

Argentiometric Method, Standard Methods; 15th Edition, pg. 270 Sec.407A

Digestion/AA*, Standard Methods; 15th Edition, pg. 157 sec $303 \mathrm{C}$

Cold Vapor Emission, Standard Methods; 15th Edition, pg. $164 \mathrm{Sec}$.303F Hydride System, Standard Methods; 15th Edition, pg. 160 Sec. $303 \mathrm{E}$ Digestion/AA*, Standard Methods; 15th Edition, pg. 152 Sec. 303A

* AA refers to analysis by Atomic Absorption Spectroscopy using a Perkin-Elmer Model 5000 . 


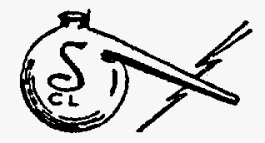


]

1

1

1

3

1

]

]

澡

औ

1

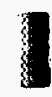

]

\section{PARAMETER}

Sodium

Copper

Lead

Nickel

Iron

Total Phosphorus

Oil and Grease

$\mathrm{pH}$

Uranium

Total Dissolved Solids

Total Organic Carbon

Aluminum

Nitrate

Total Suspended Solids

Arsenic

Nitrite

Boron

Cadmium

Chrornium

Lithium

Phenol

Sulfate

Chloride

Barium

Mercury

Selenium

silver

\section{SAMPLE ID: COMPOSITE \#1}

ANALYSIS (mg/l)

0.4

0.01

$* 0.01$

$* 0.01$

1.0

0.4

0.6

7.45

$\star 2.0$

61.0

10.0

0.6

0.32

2.0

$\star 0.001$

$* 0.1$

$* 0.1$

$\star 0.01$

$\star 0.01$

$\star 0.01$

$\star 0.1$

3.0

3.5

$* 0.01$

$\star 0.0001$

$\star 0.001$

$\star 0.01$

* Denotes less than

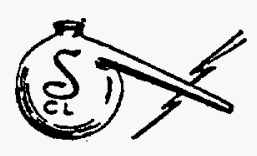




\section{SAMPLE ID: COMPOSITE \#2}

\section{PARAMETER}

Sodium

Copper

Lead

Nickel

Iron

Total Phosphorus

Oil and Grease

$\mathrm{pH}$

Uranium

Total Dissolved Solids

Total Organic Carbon

Aluminum

Nitrate

Total Suspended Solids

Arsenic

Nitrite

Boron

Cadmium

Chromium

Lithium

Phenol

Sulfate

Chloride

Barium

Mercury

Selenium

Silver

\section{ANALYSIS (mg/l)}

0.3

0.02

$\star 0.01$

$\star 0.01$

0.11

0.3

0.2

7.40

$\star 2.0$

69.0

9.0

0.1

0.29

4.0

$\star 0.001$

$\star 0.1$

$* 0.1$

$* 0.01$

$\star 0.01$

$* 0.01$

$\star 0.1$

2.0

3.2

$\star 0.01$

$\star 0.0001$

$\star 0.001$

$\star 0.01$

* Denotes less than

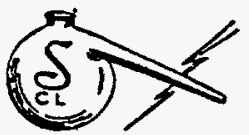




\section{SAMPLE ID: COMPOSITE \#3}

PARAMETER

Sodium

Copper

Lead

Nickel

Iron

Tota]. Phosphorus

Oil and Grease

$\mathrm{pH}$

Uranjum

Total. Dissolved Solids

Total Organic Carbon

Aluminum

Nitrate

Total Suspended Solids

Arsenic

Nitrite

Boron

Cadmium

Chromium

Lithium

Phenol

Sulfate

Chloride

Barium

Mercury

Selenium

Silver

* Denotes less than
ANALYSIS (mg/l)

1.0

0.04

$* 0.01$

$\star 0.01$

0.4

0.2

Insufficient Sample

7.40

$\star 2.0$

Insufficient Sample

7.0

$* 0.01$

4.0

Insufficient Sample

$\star 0.001$

Insufficient Sample

0.4

$\star 0.01$

0.02

$\star 0.01$

Insufficient Sample

Insufficient Sample

Insufficient sample

$\star 0.01$

$\star 0.0001$

$\star 0.001$

$\star 0.01$ 
1

1

1

].7

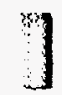

1

3

3

3

3

?

3

3

3 $\star$

Denotes less than

PARAMETER

Sodium

Copper

Lead

Nickel

Iron

Total Phosphorus

Oil and Grease

$\mathrm{pH}$

Uranium

Total Dissolved Solids

Total Organic Carbon

Alumi num

Nitrate

Total suspended Solids

Arsenic

Nitrite

Boron

Cadmium

Chromium

Lithium

Phenol

Sultate

Chloride

Barium

Mercury

Selenium

Silver

SAMPLE ID: COMPOSITE \#4

\section{ANALYSIS (mg/l)}

0,9

$* 0.01$

$* 0.01$

0.01

5.2

0.3

0.5

7.20

$\star 2.0$

60.0

20.0

$\star 0.01$

3.3

2.0

$\star 0.001$

$* 0.1$

1.3

$\star 0.01$

$* 0.01$

$\star 0.01$

Insufficient Sample

7.0

3.3

$* 0.01$

$\star 0.0001$

$\star 0.001$

$\star 0.01$

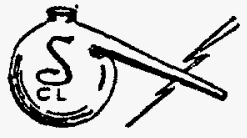


SAMPLE ID: COMPOSITE \#5

\section{PARAMETER}

Sodium

Copper

Lead

Nickel

Iron

Total Phosphorus

Oil and Grease

$\mathrm{pH}$

Uranium

Total Dissolved Solids

Total Organic Carbon

Aluminum

Nitrate

Total Suspended Solids

Arsenic

Nitrite

Boron

Cadmium

Chromium

Lithium

Phenol

Sultate

Chloride

Barium

Mercury

Selenium

Silver

* Denotes less than

\section{ANALYSIS (mg/l)}

0.6

$* 0.01$

$\star 0.01$

$* 0.01$

7.0

0.4

Insufficient Sample

6.04

$\star 2.0$

Insufficient sample

10.0

$* 0.1$

3.2

Insufficient Sample

$\star 0.001$

$\star 0.1$

$* 0.1$

$* 0.01$

$\star 0.01$

$\star 0.01$

Insufficient Sample

3.5

1.1

$\star 0.01$

$\star 0.0001$

$\star 0.001$

$\star 0.01$

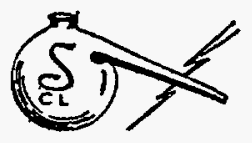


IV. OUALITY CONTROL DATA

3

3

3

3

3

3

3 
Standard samples supplied by Environmental Research Associates were analyzed along with the submitted samples. Correct answers to these standards were not known by the analyst.

RESULTS:

PARAMETER

Sodium

ERA $\$ 1995$

ERA \# 1543

$(1 / 1000)$

Copper

ERA \#9501

Lead

ERA \# 9501

Nickel

ERA \#9501

Iron

ERA \#9501

Total Phosphorus

ERA \#8801

Oil \& Grease

$\mathrm{pH}$

ERA \#8801

ERA \#1497

Total Dissolved Solids

ERA \#8801

1140.

Total Organic Carbon

ERA \#8701

Al umi num

ERA \#9501

Nitrate (as Nitrogen)

ERA \#1995

Total Suspended Solids

ERA \#8801

Arsenic

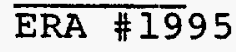

Boron

ERA \#8701

Cadmium

ERA \#1995
ACTUAL

67.0

0.60

0.207

0.163

0.213

0.129

5.4

10.0

9.0 units

9.2 units
VALUE

Concentration in (mg/l)

ACCEPTABLE

RANGE

60.0 to 74.0

0.51 to 0.69

0.190 to 0.224

0.201

0.165

0.145 to 0.181

0.193 to. 0.233

0.211

0.133

5.3

9.6

6.0 to 14.0

8.8 to 9.2 units 9.00

9.0 to 9.4 units 9.14

1040 to 1240

1160.

37.0

32.0 to 42.0

39.0

0.315

0.300 to 0.330

0.320

4.2

3.8 to 4.6

4.10

$* 1.0$

0 to 1.0

$\star 1.0$

0.035

0.025 to 0.045

0.031

0.214

0.194 to 0.234

0.202

0.050

* Denotes less than 3158 S. KOLIN AVENUE

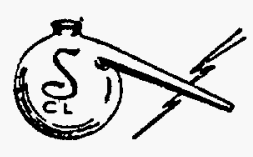


(cont.)

PARAMETER

Total Chromium

ERA \#1995

Phenol

ERA \#8801

Sulfate

ERA \#1995

ERA \#1547 (1/10)

Chloride

ERA \#8801

ERA \#8701

$(1 / 100)$

Barium

ERA \#1995

Mercury

ERA \#1995

Selenium

ERA \#1995

Silver

ERA \#I995

* Denotes less than

\begin{tabular}{lll} 
ACTUAL & ACCEPTABLE & OUR \\
VALUE & RANGE & ANALYSIS \\
\hline
\end{tabular}

$0.068 \quad 0.056$ to $0.080 \quad 0.061$

$0.130 \quad 0.110$ to $0.150 \quad 0.123$

89.0

83.0 to 95.0

5.9 to 7.7

91.0

6.7

260 .

2.81

235. to 285

2.65 to 2.97

254 .

2.75

0.376

0.340 to 0.412

0.372

0.0017

0.0009 to 0.0025

0.0015

0.021

0.010 to 0.032

0.020

0.015

0.000 to 0.030

0.013

]

* Denotes less than 


\section{Respectfully submitted,}

SCIENTIFIC CONTROL LABORATORIES, INC.

1

Fin:lls

2C

CERTIFICATION

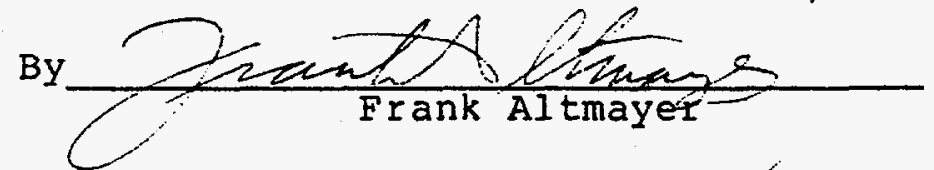
The above statements and report were subscribed and sworn to before me this TWENTIETH day of OCTOBER, 1983. 


\section{LOCKWDOD GREENE}

Planners/Engineers/Architects/Managers

Atlanta.Georgı jobname DuPont Stream Stuoy SUBJECT 7 Q 10 DATA FOR NEARBY USGS GAGNG STRCAM CHECKED BY
JOBNO. $8325 \%$. 84

SHEET NO.

DATE

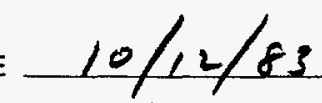

COMPUTED BY PJS

$R A$

YEAR ${ }^{+}$I DAY AVE LOW FLOW ${ }^{*}$ ORDER OF MAGNINDE RECURRENCE MTERVL

1

$73-74$

3.75

8

1.25

$74-75$

4.07

9

1.11

$75-76$

3.67

7

1.43

$76-77$

2.93

6

1.67

$77-78$

2.50

4

2.50

$78-79$

2.63

5

2.0

$79-80$

1.48

3

3.33

$80-81$

1.16

1

10.0

$81-82$

1.46

2

5.0

NOTES + YEAR IS FROM OCTOBER THRU SEPTEMBER EXCEPT 73-74 WHCH BEGAN DN MARCH $20 \mathrm{TH}$.

* average of usgs 7 day low flow for respectue year

* *ecurrence intorval (rI) $=n+1 / m$ j where $n=\#$ of veARS * $m=$ order of maONITUDE 
$9-179 b$

Extreme log data plot
April 1955
UNITED STATES DEPARTMENT OF THE INTERIOR

GEOLOGLAL SURVEY

WATEA RESOURCES ONISION
APPEND, $\times \mathrm{J}$

File.

Magritude and trequency of USGS GAGING STATION on TIM'S BRANCA

Drainage area 17,5 . sq. mi. Period $1973-1982$

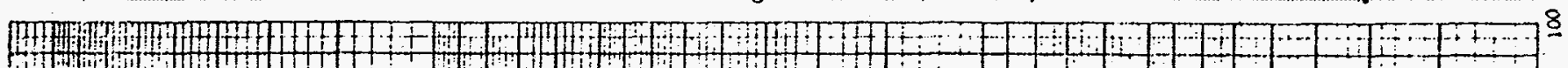

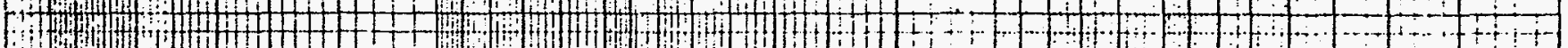

17t

TI

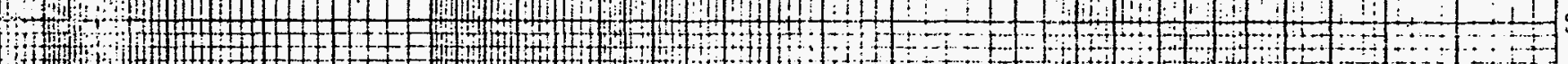

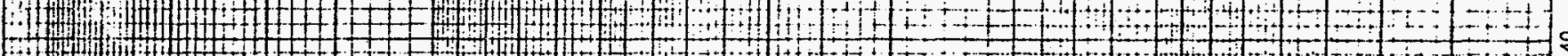

It

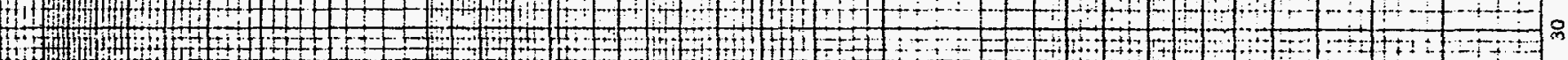

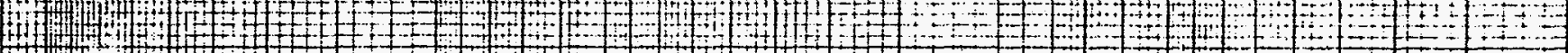

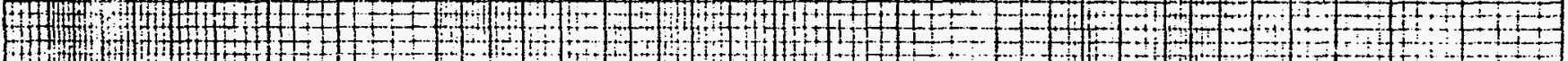

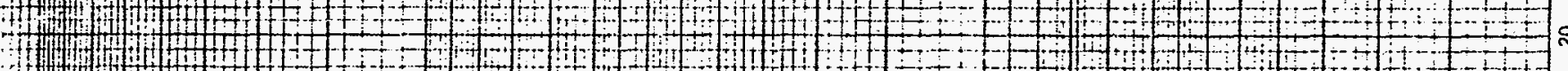

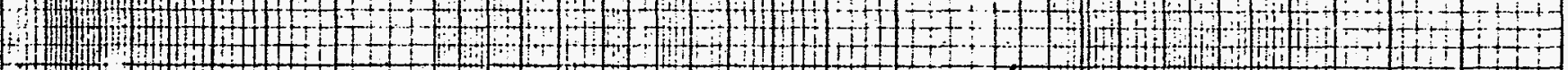

1.

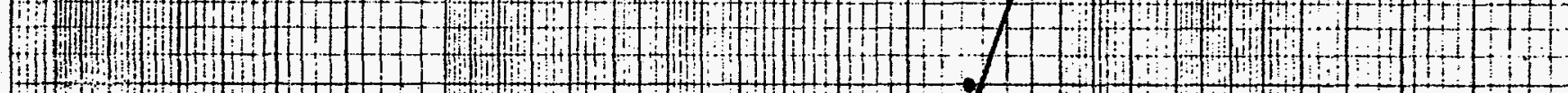

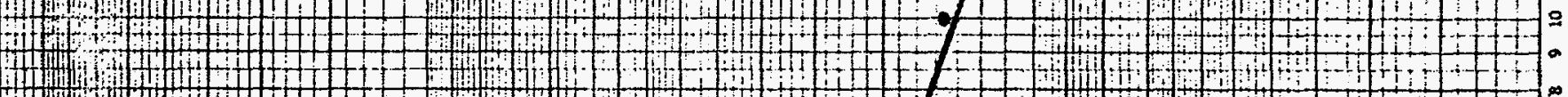

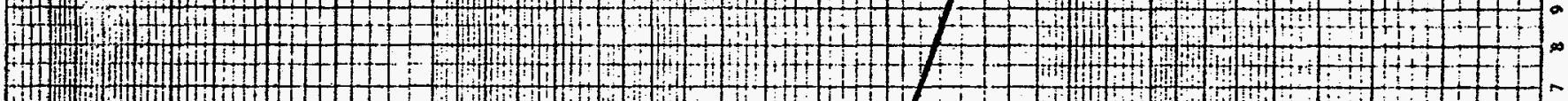

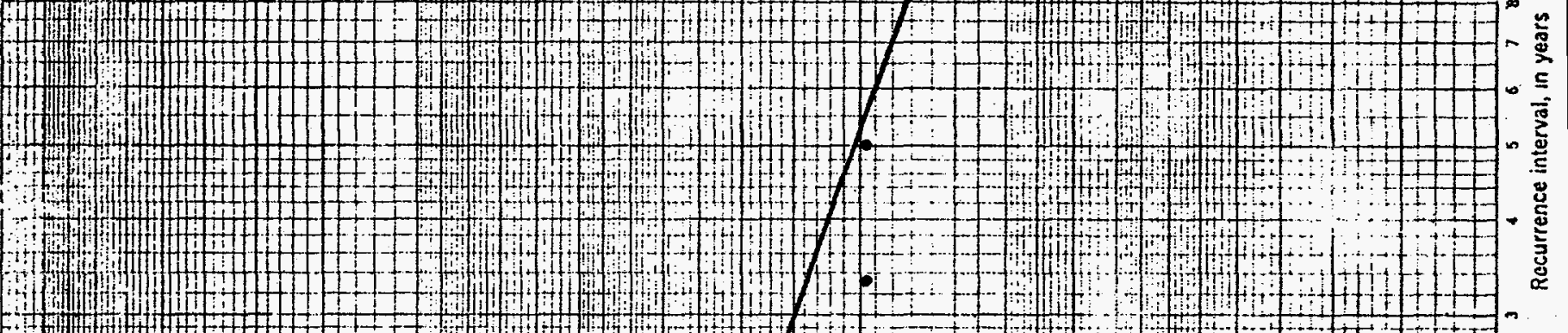

]

1

(1)

(1)

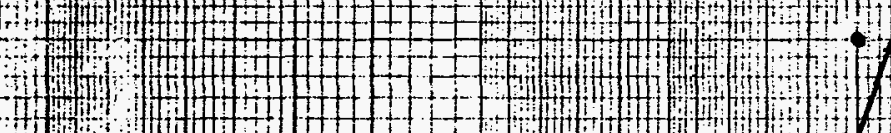

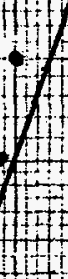

- $-1+1$

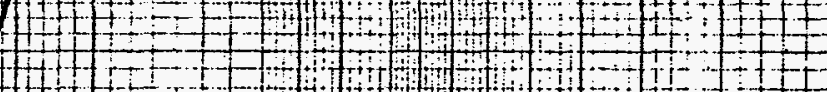

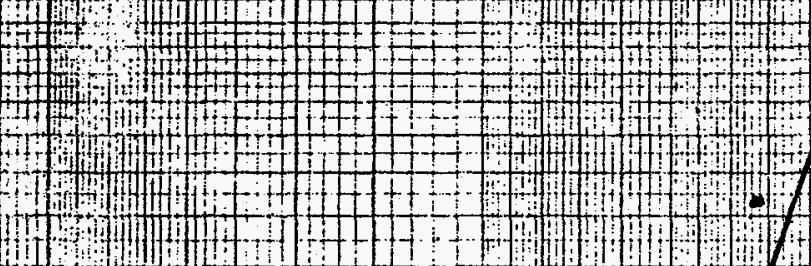

in $+\infty+1+\infty$ 


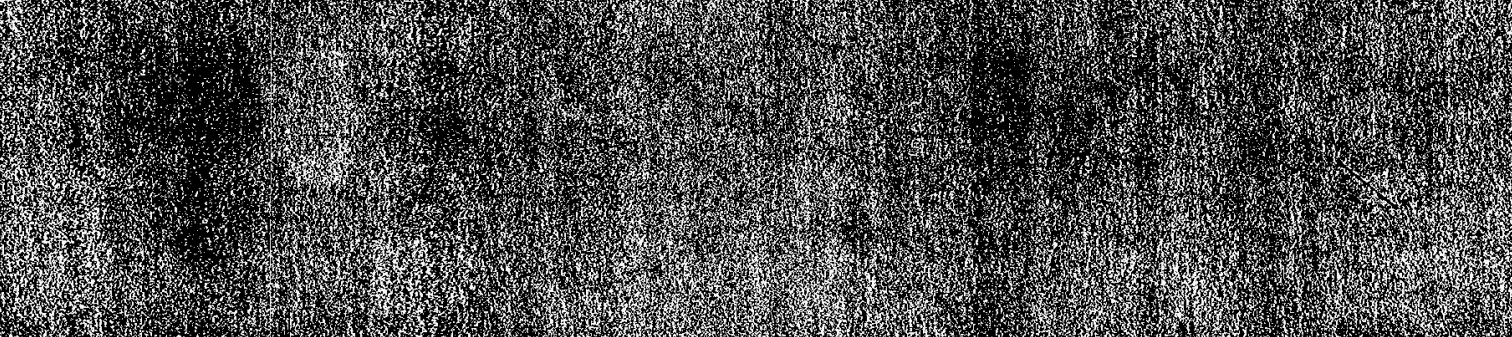

